\title{
INSURANCE-INDUCED MORAL HAZARD: A DYNAMIC MODEL OF WITHIN-YEAR MEDICAL CARE DECISION MAKING UNDER UNCERTAINTY
}

\author{
Christopher J. Cronin
}

A dissertation submitted to the faculty of the University of North Carolina at Chapel Hill in partial fulfillment of the requirements for the degree of Doctor of Philosophy in the Department of Economics.

\section{Chapel Hill}

2014

Approved by:

Donna B. Gilleskie

David K. Guilkey

Clement Joubert

Brian McManus

Helen V. Tauchen 
(C) 2014

Christopher J. Cronin

ALL RIGHTS RESERVED 


\begin{abstract}
CHRISTOPHER J. CRONIN: Insurance-Induced Moral Hazard: A Dynamic Model of Within-Year Medical Care Decision Making Under Uncertainty.

(Under the direction of Donna B. Gilleskie)
\end{abstract}

Insurance-induced moral hazard may lead individuals to overconsume medical care. Many studies estimate this overconsumption using models that aggregate medical care decisions up to the annual level. Using employer-employee matched data from the Medical Expenditure Panel Survey (MEPS), I estimate the effect of moral hazard on medical care expenditure using a dynamic model of within-year medical care consumption that allows for endogenous health transitions, variation in medical care prices, and individual uncertainty within a health insurance year. I then calculate moral hazard effects under a second set of conditions that are consistent with the assumptions of most annual decision-making models. The within-year decision-making model produces a moral hazard effect that is $24 \%$ larger than the alternative model. I also provide evidence of heterogeneous moral hazard effects, particularly between insured and uninsured individuals, and discuss related policy implications. The dissertation concludes with a counterfactual policy simulation that implements the individual mandate provision of the 2010 Patient Protection and Affordable Care Act. I find that full implementation of the individual mandate decreases the percentage of uninsured individuals in the population being analyzed from $11.8 \%$ to $6.0 \%$ and increases average medical care expenditure $77 \%$ among the newly insured. 
Without my parents, Bill and Ellen Cronin, this project would have never been started.

Without my wife, Jennifer Cronin, this project would have never been completed.

I thank each of them for their love and support. 


\section{ACKNOWLEDGMENTS}

Many individuals aided in the completion of this project. None were more important than my advisor, Donna Gilleskie, whose influence cannot be overstated. I am grateful for her effort, encouragement, and patience. I also owe a debt of gratitude to my committee members, David Guilkey, Clement Joubert, Brian McManus, and Helen Tauchen, for their advice

and support. I would also like to thank Chuck Cortemanche, Michael Darden, Randy Ellis, Michael Grossman, Matt Harris, Vijay Krishna, Tiago Pires, Dan Rees, Steve Stern, Jessica Vistnes, and participants of the UNC-Chapel Hill Applied Microeconomics Workshop for their helpful comments. It should be noted that this research was conducted at the Triangle Census Research Data Center. Support from lab administrator Bert Grider and the Agency for Healthcare Research and Quality (AHRQ) is acknowledged. The results and conclusions in this dissertation are my own and do not indicate concurrence by AHRQ or the Department of Health and Human Services. Finally, I recognize and thank Tom Cooper, who forever changed my life and career (possibly even for the better) by introducing me to the study of Economics. 


\section{TABLE OF CONTENTS}

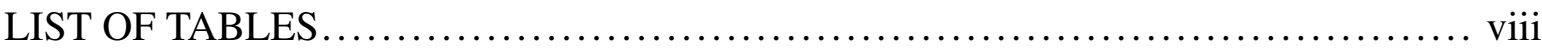

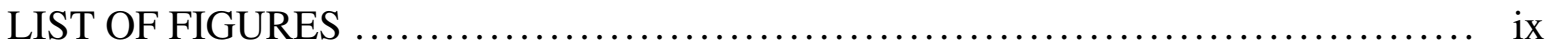

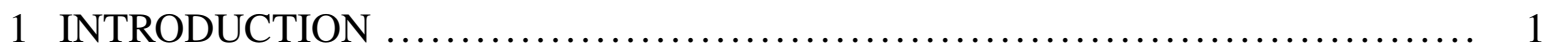

2 MOTIVATION AND BACKGROUND $\ldots \ldots \ldots \ldots \ldots \ldots \ldots \ldots \ldots \ldots \ldots \ldots \ldots \ldots \ldots \ldots, 6$

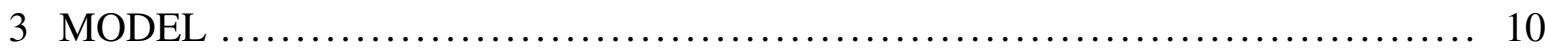

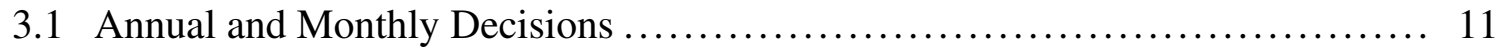

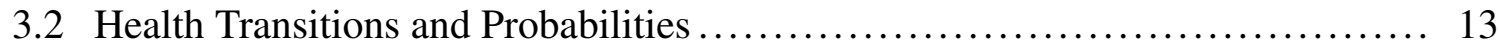

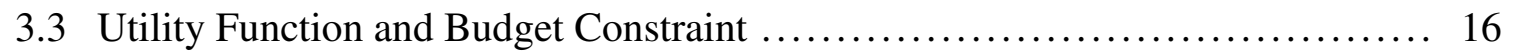

3.4 Medical Care Prices and Expenditure .............................. 17

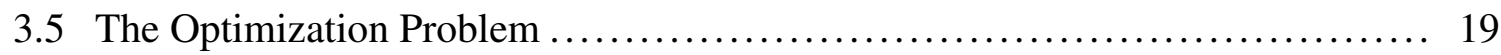

3.5.1 The Optimal Monthly Decision Rule ............................ 19

3.5.2 The Optimal Annual Decision Rule .............................. 21

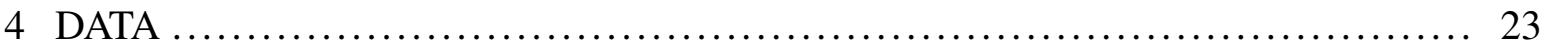

4.1 Determination of the Sample.................................... 24

4.2 Sample Statistics ............................................. 26

4.3 Prescription Drugs .............................................. 29

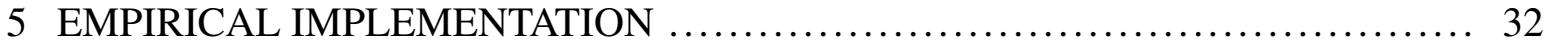

5.1 Approximating the Future Value of a Medical Care Alternative .............. 32

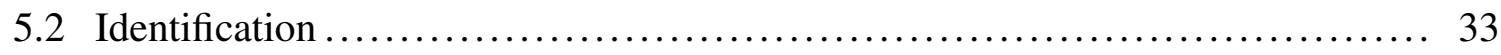

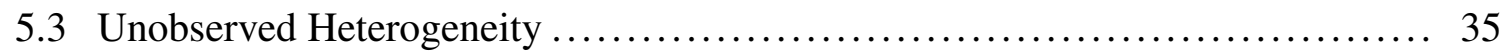

5.4 Estimation Procedure ............................................ 36 


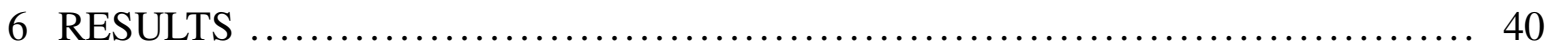

6.1 Parameter Estimates ................................................ 40

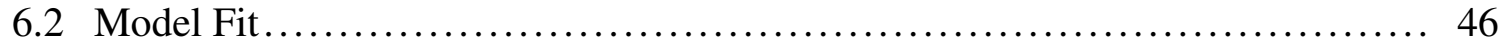

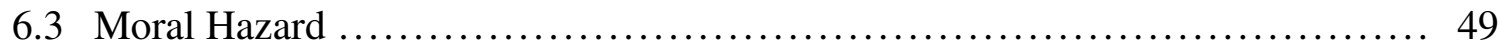

6.3.1 Moral Hazard and Modeling Assumptions ......................... 53

6.3.2 Moral Hazard and Insurance Status ............................ 58

6.4 Counterfactual Experiment: An Individual Health Insurance Mandate.......... 61

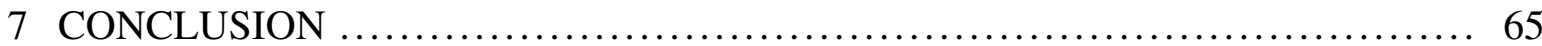

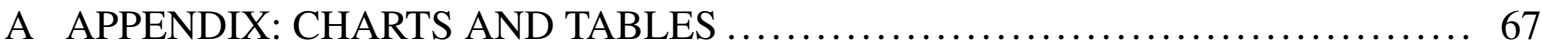

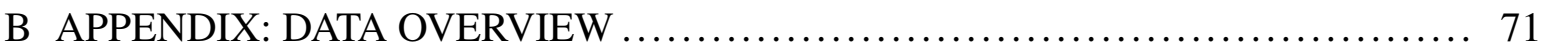

B.1 Demographic Variables............................................ 71

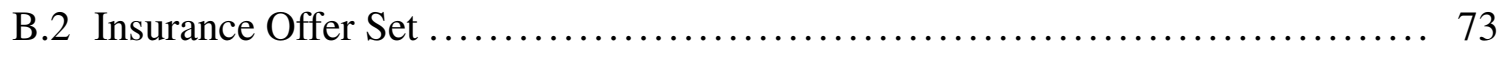

B.2.1 Logical Imputation ........................................... 74

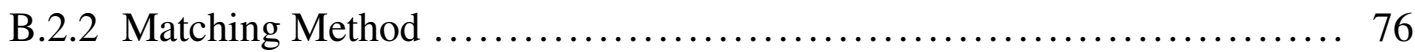

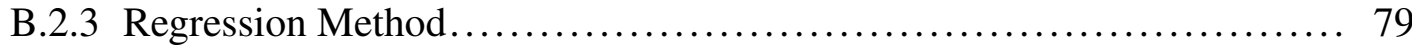

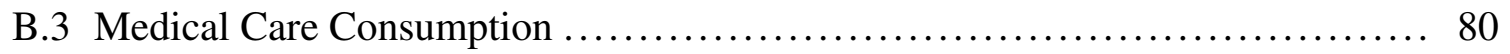

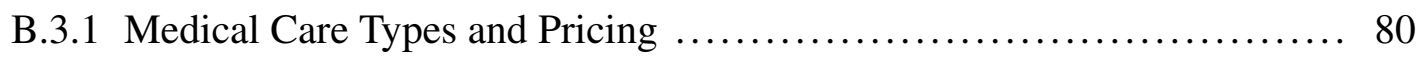

B.3.2 Consumption Dates ........................................ 82

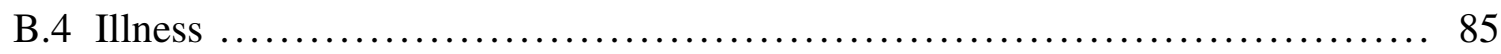

B.4.1 Classification of ICD-9-CM Codes ............................. 86

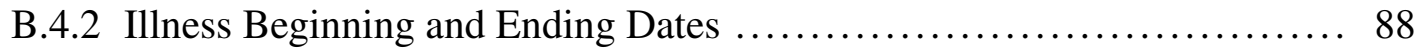

C APPENDIX: OUT-OF-POCKET EXPENDITURE EQUATION ................. 91

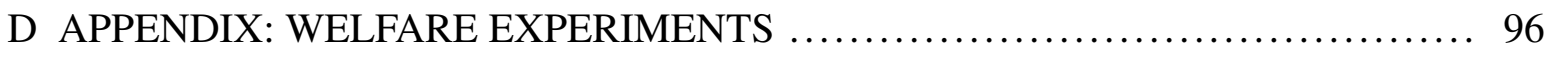

D.1 The Welfare Implications of Insurance Possession ....................... 96

D.1.1 Welfare Gains from Risk Protection ............................ 97

D.1.2 Welfare Losses from Moral Hazard ............................. 98

D.2 The Welfare Implications of Limited Insurance Choice ..................... 101 


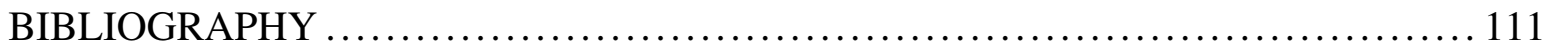




\section{LIST OF TABLES}

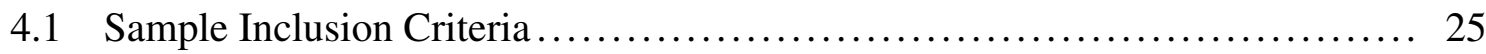

4.2 Sample Statistics by Insurance Status ............................ 27

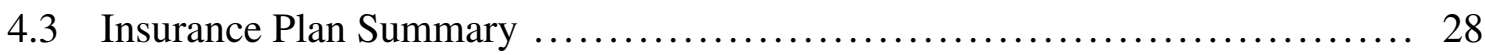

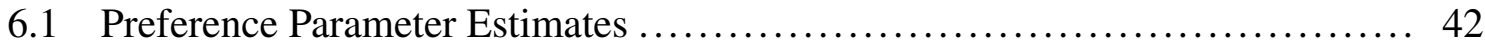

6.2 Permanent Unobserved Heterogeneity Parameter Estimates ................ 43

6.3 Illness Probability Parameter Estimates .......................... 44

6.4 General Health Status Probability Parameter Estimates.................. 45

6.5 Observed and Simulated Outcomes ............................... 48

6.6 Observed and Simulated Annual Consumption....................... 49

6.7 Predicted Effect of Insurance Possession on Medical Care Expenditure ........ 50

6.8 Other Measures of the Effects of Moral Hazard ......................... 52

6.9 Characteristics of the Insured and Uninsured $\ldots \ldots \ldots \ldots \ldots \ldots \ldots \ldots \ldots \ldots$

6.10 Predicted Health Insurance Coverage Rate $\ldots \ldots \ldots \ldots \ldots \ldots \ldots \ldots \ldots \ldots \ldots . \ldots \ldots$

A.1 Representativeness of the Sample .............................. 67

A.2 Structural Price Parameter Estimates.............................. 68

A.3 Initial Condition Probability Parameter Estimates $\ldots \ldots \ldots \ldots \ldots \ldots \ldots \ldots \ldots$

A.4 Closing Function Structural Parameter Estimates $\ldots \ldots \ldots \ldots \ldots \ldots \ldots \ldots \ldots \ldots . \quad 70$

D.1 Individual Response to Additional Plans............................. 104 


\section{LIST OF FIGURES}

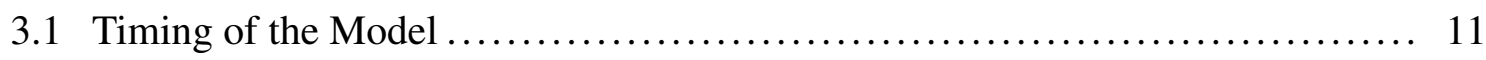

6.1 Annual Medical Care Expenditure $\ldots \ldots \ldots \ldots \ldots \ldots \ldots \ldots \ldots \ldots \ldots \ldots \ldots \ldots . \ldots \ldots$

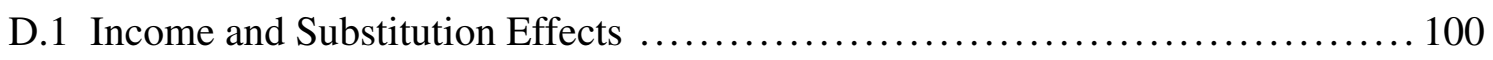




\section{INTRODUCTION}

Economic theory suggests that health insurance may increase medical care consumption above the socially optimal level (Arrow 1963; Pauly 1968). The incentives that elicit this increase in consumption are often referred to as moral hazard (Cutler and Zeckhauser 2000). ${ }^{1}$ Empirical studies tend to estimate moral hazard effects using models that aggregate medical care decisions up to the annual level. In this dissertation, I study insurance-induced moral hazard using a dynamic stochastic model of within-year medical care consumption decisions. The within-year decision-making model more accurately captures the data generating process by relaxing several assumptions made frequently in the literature. Specifically, the model allows for endogenous health transitions, variation in medical care prices, and individual uncertainty within a health insurance year. The research contributes to the literature in two ways. First, I show that the within-year decision-making model produces a moral hazard effect that is $24 \%$ larger than an alternative model that imposes the more restrictive assumptions of a typical annual expenditure model. ${ }^{2}$ Second, I provide evidence of heterogeneous moral hazard effects, particularly between insured and uninsured individuals. I explain why each of these findings is consistent with economic theory and show how differences in estimated moral hazard effects can lead to large differences in predicted policy outcomes. I also conduct a series of counterfactual policy simulations to study the potential effects of the individual insurance mandate provision of the 2010 Patient Protection and Affordable Care

\footnotetext{
${ }^{1}$ The term moral hazard is used rather loosely in the health economics literature. I describe moral hazard as the incentives associated with insurance possession that lead to changes in individual behavior. I focus on one effect of moral hazard, which is the effect that health insurance possession has on medical care consumption. I discuss the welfare implications of this additional consumption in Appendix D.

${ }^{2}$ The moral hazard effect is measured as the percentage increase in mean annual medical care expenditure that is caused by insurance acquisition. A more detailed description of this calculation is given in Section 6.3.
} 
Act (ACA).

The within-year decision-making model is motivated by theoretical (Grossman 1972; Keeler, Newhouse, and Phelps 1977) and empirical (Gilleskie 1998; Cardon and Hendel 2001; Khwaja 2001, 2010; Blau and Gilleskie 2008) models of health production and medical care demand. An individual's optimization problem consists of an annual health insurance decision, followed by a sequence of monthly medical care consumption decisions made over the course of a health insurance year. I model monthly medical care decisions to allow the unique benefits and costs associated with the timing of unexpected illness and potential medical care consumption to impact behavior within the model. Within each month, a forward-looking individual responds to an endogenous stochastic health event by consuming units of medical care. The (anticipated) primary benefit of medical care consumption is improved future health. The (anticipated) primary cost is financial (i.e., a decrease in the current consumption of non-medical goods). ${ }^{3}$ When health insurance has dynamic cost-sharing features (i.e., deductible and stop loss), an additional benefit of current medical care consumption is the reduced cost of future care once accumulated expenditure crosses a threshold. ${ }^{4}$ The model also allows a direct contemporaneous utility benefit or cost of medical care consumption that is independent of the productive and financial effects.

An individual faces uncertainty along multiple dimensions of the optimization problem. Prior to both an annual health insurance decision and each monthly medical care decision, an individual is uncertain of his future health outcomes, medical care consumption, and medical care prices. Furthermore, I assume that prior to medical care consumption an individual knows the conditional distributions from which medical care prices are drawn, but does not

\footnotetext{
${ }^{3}$ I qualify anticipated benefits and costs because the estimated model parameters determine both the sign and magnitude of medical care productivity (in producing positive health outcomes) and the disutility from reduced consumption of non-medical goods. I expect to find that medical care is productive, a reduction in non-medical consumption yields disutility, and that each plays a principle role in the medical care decision-making process.

${ }^{4} \mathrm{~A}$ deductible is a fixed amount of accumulated medical care expenditure that must be reached (within an insurance year) before the insurer covers any part of total medical care costs. A stop loss is an accumulated out-of-pocket expenditure threshold at which an individual's share of the total cost of any additional medical care consumed during that insurance year is zero.
} 
know the exact prices he will be charged for different types of care. ${ }^{5}$ Though it is typically assumed that prices are known prior to consumption, there are several reasons why price uncertainty is a more realistic assumption. First, an individual rarely knows a physician's diagnosis and recommended treatment prior to an office or hospital visit. Second, in the U.S., medical care providers do not display a menu of prices and there is evidence of wide price variation in local medical care markets. Each of these market characteristics make it difficult for an individual to know exactly how much he will be charged for care.

The within-year decision-making model, which is characterized by these important market features, also has several empirical advantages. First, the model has the ability to capture patterns in the data that are explained by within-year behavior. For example, in the estimation sample, average monthly medical care expenditure is $\$ 127.60$ higher in months where an individual has an acute illness. This spending gap exists even when conditioning on chronic illness entering the year: expenditure is $\$ 119.78$ higher in acute illness months for those without a chronic illness and $\$ 140.30$ higher for those with a chronic illness. ${ }^{6}$ This behavior can be explained by the within-year decision-making model if medical care decreases the likelihood of having an acute illness and has a financial cost. Second, the assumptions imposed on the within-year decision-making model may impact the estimated effect of moral hazard. For example, the model allows medical care consumption to affect health over the course of a health insurance year (i.e., within-year health transitions are treated as endogenous). Most models of medical care demand either do not model health at all (implicitly assuming that health transitions are exogenously determined) or model annual health outcomes. Allowing for endogenous health transitions within an insurance year impacts the estimated effect of moral hazard if the insured consume more medical care but then find themselves in better health, decreasing the need for medical care consumption in the future.

\footnotetext{
${ }^{5}$ I use the terms price and total cost, interchangeably, to describe the total amount billed for a unit of medical care. The out-of-pocket cost to an individual is often less than the price billed due to insurance cost-sharing. The difference between medical care prices and costs paid out-of-pocket is discussed further in Section 3.4.

${ }^{6}$ Throughout the dissertation, all dollar amounts are reported in 1996 dollars, unless stated otherwise.
} 
I estimate the model parameters via maximum likelihood using employer-employee match data from the 1996-1999 Medical Expenditure Panel Survey (MEPS). I use simulation techniques to examine model fit and to calculate the effect of moral hazard on medical care expenditure. I conduct a set of counterfactual simulations to study how different assumptions imposed on a within-year decision-making model lead to different moral hazard effects. Specifically, I examine how the estimated effect of moral hazard responds to the assumption that health transitions are exogenously determined (assumption 1), that medical care prices are known prior to consumption (assumption 2), and that all health and price shocks are known at the beginning of the year (assumption 3). The main counterfactual imposes assumptions 1,2 , and 3 , as these assumptions are consistent with those made implicitly in most annual expenditure models, such as Cardon and Hendel (2001), Einav, Finkelstein, Ryan, Schrimpf, and Cullen (2013), and Kowalski (2013). ${ }^{7}$

Health insurance is predicted to increase mean annual medical care expenditure by $92 \%$ using the (preferred) within-year decision-making model and 74\% when assumptions 1,2, and 3 are imposed. The counterfactual estimate compares favorably with estimates produced by several annual expenditure models in the literature. Ultimately, the presence of health and price uncertainty at the time of medical care consumption in the preferred model decreases the expected value of medical care. The larger moral hazard effect produced by the withinyear model is driven by exceptionally low medical care consumption when uninsured, as risk averse individuals who face uncertainty are exposed to significant risk in consumption.

I find heterogeneous moral hazard effects across the population. The $92 \%$ increase in mean annual medical care expenditure that results from insurance acquisition is driven by individuals with exceptionally large increases in expenditure. If the top $1 \%$ of additional spenders are dropped, then the increase in mean expenditure due to insurance is reduced to

\footnotetext{
${ }^{7}$ Bajari, Hong, Khwaja, and Marsh (2013) is similar but does allow for some uncertainty at the time of medical care decision making. The authors assume that an individual selects his total annual medical care expenditure while knowing only the distribution of the proportion of that expenditure he must pay out-of-pocket.
} 
$65 \%$. Furthermore, $44 \%$ of the population does not increase their expenditure at all when they become insured. I also find significant differences in how insured and uninsured individuals respond to coverage. When individuals are moved from an uninsured state to their optimal plan, mean annual medical care expenditure in the insured population increases by $96 \%$; however, mean annual medical care expenditure in the uninsured population increases by only 55\%. In Section 6.3.2, I discuss the factors that drive these differences and explore the policy implications of the differential response to coverage between these two groups.

The dissertation concludes with a counterfactual exercise that examines the behavioral response to an individual insurance mandate that is consistent with the ACA. ${ }^{8}$ When facing a penalty of $\$ 695$ (in 2016 dollars) or $2.5 \%$ of income (whichever is larger) for failing to carry health insurance coverage, the proportion of the population being analyzed that chooses to be uninsured decreases from $11.8 \%$ to $6.0 \%$. Of the previously uninsured population, mean annual medical care expenditure for the newly insured increases by $77 \%$ (moral hazard effect), while expenditure for those remaining uninsured falls by $2.4 \%$ (income/penalty effect). Given that the full implementation penalty does not elicit universal coverage, I also examine the welfare implications of forced insurance take-up. Holding insurance premiums and medical care prices fixed, I find that among uninsured individuals the average expected welfare loss from forced take-up is $\$ 1608$ (2016 dollars). ${ }^{9}$

The following section provides motivation for this research and discusses some of the previous literature. Section 3 details the theoretical model of insurance and within-year medical care demand. Section 4 describes the data and the sample used in estimation. Section 5 details the estimation procedure and discusses identification. Sections 6 presents parameter estimates, model fit, and counterfactual simulations. Section 7 concludes.

\footnotetext{
${ }^{8}$ This counterfactual implements the individual mandate provision of the ACA only. There are many other regulations that the ACA imposes upon the marketplace that are not considered. Furthermore, the empirical analysis conducted in this research focuses on a population of individuals who are unmarried, childless, employed, between the ages of 19 and 64, and have the ability to purchase health insurance through their employers.

${ }^{9}$ The average expected welfare loss is measured as the average penalty that would make an uninsured individual indifferent between remaining uninsured and paying the penalty or being insured and paying the premium.
} 


\section{MOTIVATION AND BACKGROUND}

Health insurance generates welfare by protecting risk averse individuals from medical expenses associated with unforeseen health shocks (Arrow 1963). However, the welfare gains from risk protection are potentially mitigated by changes in individual behavior after becoming insured. For example, insurance lowers the out-of-pocket cost of medical care, which can lead to excess consumption when sick, known as ex-post moral hazard (Pauly 1968). Also, a reduction in the expected cost of curative medical care can reduce participation in healthy behaviors (e.g., preventative medical care, diet, exercise, etc.) leading to worse health outcomes and potentially greater medical care consumption in the future, known as ex-ante moral hazard (Cutler and Zeckhauser 2000). ${ }^{10}$ Each of these forces drives insured individuals to consume medical care past the socially optimal level, generating a welfare loss. ${ }^{11}$ Therefore, efficient health insurance plan design requires an understanding of how health insurance leads to changes in individual medical care consumption behavior.

\footnotetext{
${ }^{10}$ In the empirical health economics literature, moral hazard normally refers only to ex-post moral hazard (some exceptions are Dave and Kaestner (2009) and Kelly and Markowitz (2009)). Ex-ante moral hazard is difficult to study for two reasons. First, ex-ante moral hazard involves changes in many non-medical behaviors (i.e., exercise, diet, smoking, etc.). Second, ex-ante moral hazard results from poor health behaviors that lead to worse health outcomes, meaning endogenous health transitions must be modeled. The model presented in this research allows for ex-post moral hazard and limits the effect of ex-ante moral hazard to changes in medical care consumption. That is, an individual in the model may respond to health insurance coverage by consuming medical care less frequently, which may lead to poor health outcomes and greater medical care consumption in the future. However, I do not model non-medical behavioral responses to insurance acquisition.

${ }^{11}$ It is assumed here that the level of medical care consumption when uninsured is socially optimal. If medical care prices are non-competitive or if their are externalities in medical care consumption (e.g., the reduction of communicative diseases), then consumption when uninsured may not be efficient. Even if the level of consumption when uninsured is socially optimal, it is not the case that the full increase in medical care consumption due to insurance acquisition is welfare reducing. As is covered extensively by Nyman (1999a,b,c), the additional medical care consumption that is caused by insurance possession results from both lower out-of-pocket medical care costs and an income transfer from the well to the sick, the latter of which is welfare neutral. The main moral hazard effect discussed in this research includes the income effect, because there are no welfare calculations presented at this time.
} 
Determining how medical care consumption and welfare are affected by health insurance has been a central focus of empirical health insurance and medical care research over the past 30 years. The primary challenge in estimating, for instance, the percentage increase in mean annual medical care expenditure that is caused by health insurance possession (i.e., a measure of the effect of moral hazard) is the endogenous selection of health insurance. Those who expect to consume more medical care during a health insurance year select generous health insurance coverage; this is known as adverse selection (Akerlof 1970). Both moral hazard and adverse selection lead to a positive correlation between observed medical care expenditure and insurance possession/generosity; however, the extent to which this correlation is driven by moral hazard or adverse selection has important policy implications. ${ }^{12}$

One method that has been used to control for endogenous insurance selection, so that moral hazard effects can be identified, is a randomized experiment. A well known example is the RAND Health Insurance Experiment (HIE). The 1971 RAND HIE was a multiyear, \$295 million (in 2011 dollars, (Greenberg and Shroder 2004)) medical care study that, among other things, randomly distributed health insurance plans to participants in 6 U.S. cities and recorded health and medical care consumption in the years following (for more details, see Newhouse 1974, 1993). By randomly assigning coverage, the experiment's design created exogenous variation in insurance holdings so that price elasticities (i.e., another measure of the effect of moral hazard) could be estimated. A more recent example of researchers using experimental data to study moral hazard is the Oregon HIE. In 2008, the Oregon Health Authority expanded the state's Medicaid program to 10,000 additional lowincome adults using a lottery (i.e., qualifying individuals were randomly selected and given the ability to apply for coverage). Again, random assignment allows these researchers to study moral hazard without concern for endogenous insurance selection. This experiment

\footnotetext{
${ }^{12}$ If moral hazard is the dominant force behind this correlation, then policy makers should encourage less risk protection through greater cost-sharing. Requiring an individual to pay a larger share of the price of medical care reduces his incentive to overconsume care. If adverse selection is the dominant force, then policy makers should encourage greater risk pooling.
} 
is ongoing, though one year (Finkelstein, Taubman, Wright, Bernstein, Gruber, Newhouse, Allen, Baicker, and the Oregon Health Study Group 2012) and two year (Baicker, Taubman, Allen, Bernstein, Gruber, Newhouse, Schneider, Wright, Zaslavsky, Finkelstein, and the Oregon Health Study Group 2013) evaluations of the program have been published. There are also numerous quasi-experimental studies that use econometric techniques and exogenous (or near exogenous) shifts in insurance policy, such as Medicaid expansion (Currie and Gruber 1996; Dafny and Gruber 2005) or the Massachusetts market reforms (Miller 2012; Kolstad and Kowalski 2012), to control for adverse selection.

While both experimental and quasi-experimental techniques have been used to successfully control for adverse selection so that moral hazard effects may be identified, a principle goal in this literature is to move beyond measuring the spending response to observed plans and/or policies. ${ }^{13}$ Recently, research efforts have focused on measuring the welfare implications of the additional spending caused by moral hazard and designing insurance plans and insurance plan alternative sets that improve consumer welfare. In this pursuit, researchers have turned to structural modeling. ${ }^{14}$ Importantly, structural models have allowed researchers to both control for adverse selection in order to quantify moral hazard effects and to calculate the welfare implications of these effects. Furthermore, because insurance decisions are typically modeled and insurance cost-sharing characteristics are allowed to impact optimal

\footnotetext{
${ }^{13}$ The experimental and quasi-experimental research found in this literature has generally focused on analyzing the effects of specific policies on outcomes of interest. Unfortunately, these results are difficult to generalize, as the estimated effects are normally applicable for only a specific policy and population. Manning, Newhouse, Duan, Keeler, and Leibowitz (1987) and Keeler and Rolph (1988) are exceptions to this rule, as they estimate a single price/co-insurance elasticity of medical care demand that is frequently used by researchers and policy makers to predict changes in medical care expenditure levels that would result from insurance plans and policies not observed in the marketplace. However, as is discussed extensively in Aron-Dine, Einav, and Finkelstein (2013), application of the single price elasticity measure requires a researcher to characterize a health insurance plan by a single price. Because modern health insurance plans are characterized by many cost-sharing features that change the out-of-pocket cost of medical care over the course of a health insurance year, there is no obvious way to summarize a plan by a single price. Aron-Dine et al. (2013) conduct an empirical exercise where they predict medical care expenditure using the single price elasticity and implement several common strategies for determining a single price. Their results show wide variation in predicted results depending on the strategy used.

${ }^{14}$ In this context, structural modeling should be interpreted as explicitly modeling and estimating the parameters of an individual's optimization problem with regard to medical care consumption. See Chiappori and Salanie (2002) and Einav, Finkelstein, and Levin (2010) for a review of this technique.
} 
medical care decision making through the budget constraint, the models are well suited to study behavioral and welfare responses to counterfactual insurance plans, insurance alternative sets, and regulatory policies.

Among the related structural models that have been designed and estimated (Cardon and Hendel 2001; Khwaja 2001, 2010; Einav et al. 2013; Kowalski 2013; Bajari et al. 2013; Handel 2013), all have aggregated medical care expenditures and health outcomes up to the annual level. ${ }^{15}$ Annual expenditure models have been popular, here and elsewhere in the health economics literature, primarily due to data limitations. Annual medical care expenditure data are accessible. Large public data sets, which contain total annual expenditure variables that have been cleaned and are ready for immediate use, are used by many empirical researchers and allow for nationally representative findings. ${ }^{16}$ Also, estimation of annual expenditure models can be achieved without high frequency explanatory data, such as illness state, which is both difficult to find and desirable when estimating a model of within-year medical care decisions. ${ }^{17}$ My research builds on these structural annual expenditure models by allowing for monthly medical care consumption decisions to be made over a health insurance year and by relaxing several assumptions commonly made in annual decision-making models. ${ }^{18}$

\footnotetext{
${ }^{15}$ Khwaja $(2001,2010)$ aggregates to the biennial level.

${ }^{16}$ Examples are: the Medical Expenditure Panel Survey (MEPS); the Health and Retirement Survey (HRS), which has been cleaned by RAND; and the Medicare Current Beneficiary Survey (MCBS).

${ }^{17}$ Note that while insurer claims data (or claims data from a large self-insuring company, which are used in Einav et al. 2013; Kowalski 2013; Bajari et al. 2013; and Handel 2013) allow for the observation of highfrequency medical care consumption decisions, illness state is only observed when an individual chooses to consume care. Therefore, endogenous health transitions cannot be modeled well using claims data.

${ }^{18} \mathrm{~A}$ few researchers have studied health insurance and/or medical care demand using within-year behavior as an outcome. Keeler and Rolph (1988) and Keeler, Buchanan, Rolph, Hanley, and Reboussin (1988) use data from the RAND Health Insurance Experiment to examine medical care consumption during treatment episodes in order to study the role of insurance deductibles and stop losses. Aron-Dine, Einav, Finkelstein, and Cullen (2012) use quasi-experimental data to "investigate whether individuals exhibit forward-looking behavior in their response to the non-linear pricing common in health insurance contracts." Ellis (1986) models demand for mental health services in the first 30,60, and 90 days of a year as a function of expected end of the year prices, while assuming insurance coverage is determined exogenously. Gilleskie (1998) estimates a structural model of daily medical care consumption and absenteeism during acute illness episodes, also assuming insurance coverage is determined exogenously. Despite the smaller units of behavior, these authors can separately identify adverse selection and/or moral hazard type effects only by using (quasi-)experimental data or simplifying assumptions.
} 


\section{MODEL}

This section describes the optimization problem solved by an unmarried, childless, employed individual who makes an annual health insurance decision followed by a sequence of medical care consumption decisions to maximize the value of his expected discounted future utility. ${ }^{19}$ The timing of the model can be observed in Figure 3.1. At the beginning of each year, $y$, a forward-looking individual observes the set of health insurance alternatives offered by his employer, his general health status, and the presence of any illnesses. Before the start of the first month, $t=1$, he chooses the health insurance alternative that maximizes his expected discounted future utility. Among other things, this expected utility is a function of anticipated medical care behavior within the year conditional on insurance coverage. In this research the within-year medical care behavior is modeled explicitly.

At the beginning of each month, an individual learns his illness state, which evolves stochastically over the course of the year and is influenced by his general health status, illness history, and previous medical care consumption. After learning his current illness state, the individual decides how much (and what types of) medical care to consume. The amount he pays for a unit of medical care depends on the unit price, the cost-sharing characteristics of his health insurance plan, and his accumulated medical care expenditure within the coverage year. Much like the price uncertainty individuals face in the US medical care market, the total price of care is stochastic over time and unknown prior to consumption. After making a

\footnotetext{
${ }^{19}$ The model features employed individuals who receive an employer-sponsored health insurance offer (ESHI) because health insurance information is only available for these individuals in the data. ESHI is the most popular mechanism by which individuals obtain health insurance in the United States. Of the non-elderly population in 2011: 55.8\% held ESHI, 18\% were uninsured, $20.5 \%$ were insured by state or federal governments, and 5.7\% were privately insured (Kaiser Family Foundation). The model focuses on single, childless individuals in order to explicitly capture dynamic health, non-linear out-of-pocket prices, and medical care demand throughout the insurance coverage period. The estimation sample includes men and non-pregnant women.
} 
Figure 3.1: Timing of the Model

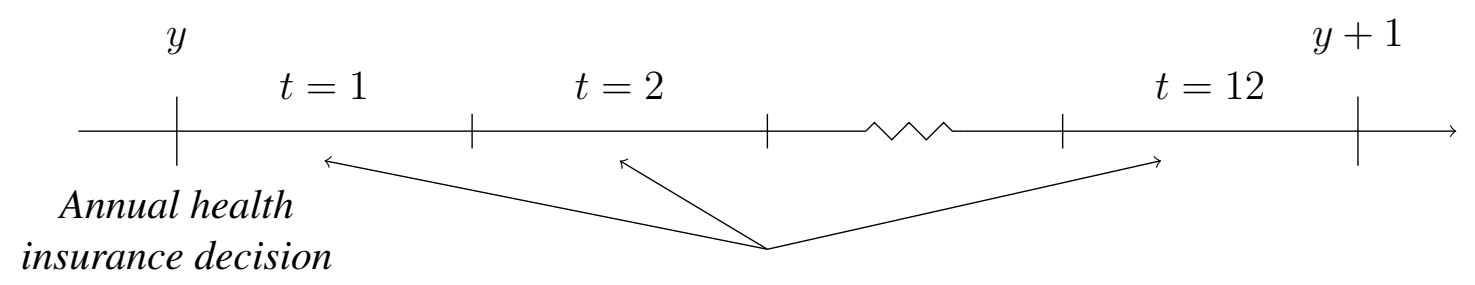

Monthly medical care decisions

Each month:

(1) Learn illness state

(2) Select among medical care alternatives

(3) Observe updated general health status

medical care decision, the individual's general health status evolves prior to the next month. The remainder of this section explains the model and solution in greater detail.

\subsection{Annual and Monthly Decisions}

At the beginning of each year, an individual observes the set of health insurance plans available to him from his employer. Each plan is defined by its premium, network type, and a set of cost-sharing characteristics. The cost-sharing features enter an individual's budget constraint throughout the year, determining how much is paid out-of-pocket for medical care. The following plan characteristics enter the model: out-of-pocket premium, composite annual deductible, doctor's office deductible, hospital deductible, stop loss, hospital co-insurance rate, hospital co-pay level, doctor's office co-insurance rate, doctor's office co-pay level, prescription drug co-insurance, and the extent to which the plan restricts coverage to a network of 
physicians (HMO, PPO, or FFS). ${ }^{20}$ An indicator function, $I_{i y}^{j}$, equals one if individual $i$ selects insurance plan $j$ in year $y$ and zero otherwise. ${ }^{21}$ Only one plan can be held at a time, so that

$$
\sum_{j \in J^{i}} I_{i y}^{j}=1 \quad \forall i \forall y
$$

where $J^{i}$ is the set of exogenously determined employer-sponsored health insurance (ESHI) plans and includes the option to decline all plans. ${ }^{22}$

In each month, an individual learns his illness state (defined below) before making a medical care consumption decision. He chooses the number of doctor visits, $v_{i t}$; hospital days, $s_{i t},{ }^{23}$ and whether or not to consume prescription drugs, $r_{i t}{ }^{24}$ The monthly medical care decision is represented by an indicator function, $d_{i t}^{v s r}$, that equals one if an individual chooses the bundle $(v, s, r)$ and zero otherwise. Bundles are mutually exclusive with a maximum of

\footnotetext{
${ }^{20} \mathrm{~A}$ deductible and a stop loss are described in footnote 4 . A co-insurance rate is the share of the medical care price that an individual must pay out-of-pocket (the remainder is paid by the insurer). A co-pay level is a fixed dollar amount that an individual must pay out-of-pocket for a unit of medical care (again, the remainder is paid by the insurer). A health maintenance organization (HMO) here refers to an insurance plan that limits its enrollees to receiving medical care from a specified group of providers. A preferred provider organization (PPO) is a plan that defines a preferred network of providers from which care can be purchased less expensively. If an enrollee chooses to seek care outside of this network, coverage is still provided but at a higher out-of-pocket cost. A fee-for-service (FFS) plan covers an enrollee equally at all medical care providers.

${ }^{21}$ For notational simplicity and consistency, I include the subscript $i$ to describe individual level variables only when defining the variable. The subscript $i$ is suppressed thereafter.

${ }^{22}$ Some individuals select a job based (at least partially) on the health insurance offered. However, modeling an individual's decision to accept a particular job, with health insurance options as a job characteristic, requires modeling the employment decision as a function of health insurance characteristics. Thus, this exogeneity assumption has become the norm in the literature.

${ }^{23}$ Hospital days are chosen rather than the standard (hospital) nights because inpatient and outpatient hospital visits are not modeled separately. A visit to the ER and an outpatient procedure each constitute a decision to consume one hospital day. A single overnight visit reflects a decision to consume two hospital days.

${ }^{24}$ According to the Centers for Disease Control and Prevention (CDC) these three types of care account for over $80 \%$ of personal medical care expenditure in the United States. For the population being analyzed, the percentage is even higher because individuals are non-elderly and unlikely to consume nursing home care or home healthcare. Other relevant medical care products, such as dental and optical, are unlikely to be covered by standard ESHI plans and are thus excluded from the study.
} 
$V$ doctor visits and $S$ hospital days each month, such that

$$
\sum_{v=0}^{V} \sum_{s=0}^{S} \sum_{r=0}^{1} d_{i t}^{v s r}=1 \quad \forall i \forall t
$$

\subsection{Health Transitions and Probabilities}

Three measures of individual health evolve stochastically over the course of the insurance year. The acute illness state, $A_{i t}=\{0,1\}$, and the chronic illness state, $C_{i t}=\{0,1\}$, are dichotomous. General health status, $H_{i t}$, takes on one of three values: excellent $\left(H_{i t}=2\right)$, $\operatorname{good}\left(H_{i t}=1\right)$, or poor $\left(H_{i t}=0\right) .^{25}$

I define an acute illness as any medical condition that eventually subsides and, under normal conditions, has no permanent effect on an individual's health or medical care consumption. This characterization describes both short-natured ailments, such as a common cold or influenza, as well as non-permanent but persistent conditions, such as a pneumonia or a broken bone. In estimation, the probability that an individual is in an acute illness state in month $t$ is determined by a logistic function such that

$$
P\left(A_{t}=1\right)=\pi_{t}^{1}=\frac{\exp \left(\alpha_{0}+\alpha_{1} \mathbf{W}_{\mathbf{t}}+\alpha_{2} \mathbf{H}_{\mathbf{t}}^{\mathbf{A}}+\alpha_{3} \mathbf{M}_{\mathbf{t}-\mathbf{1}}+\alpha_{4} \mathbf{N}_{\mathbf{t}}^{\mathbf{A}}+\mu_{1}^{k}\right)}{1+\exp \left(\alpha_{0}+\alpha_{1} \mathbf{W}_{\mathbf{t}}+\alpha_{2} \mathbf{H}_{\mathbf{t}}^{\mathbf{A}}+\alpha_{3} \mathbf{M}_{\mathbf{t}-\mathbf{1}}+\alpha_{4} \mathbf{N}_{\mathbf{t}}^{\mathbf{A}}+\mu_{1}^{k}\right)}
$$

where $\mathbf{W}_{\mathbf{t}}$ contains demographic factors such as sex, race, income, education, MSA indicator, age, and month indicators; $\mathbf{H}_{\mathbf{t}}^{\mathbf{A}}$ is general health status and illness state entering the month $\left(\mathbb{1}_{H_{t}<2}, \mathbb{1}_{H_{t}<1}, A_{t-1}, C_{t-1}\right) ; \mathbf{M}_{\mathbf{t}-\mathbf{1}}$ is medical care consumption in the prior month $\left(v_{t-1}, s_{t-1}, r_{t-1}\right) ; \mathbf{N}_{\mathbf{t}}^{\mathbf{A}}$ contains interactions of the variables in $\left(\mathbf{W}_{\mathbf{t}}, \mathbf{H}_{\mathbf{t}}^{\mathbf{A}}, \mathbf{M}_{\mathbf{t}-\mathbf{1}}\right)$; and $\mu_{1}^{k}$ captures unobserved permanent individual heterogeneity for an individual of type $k$, where $k=1, \ldots, K{ }^{26}$ Interactions are used to allow the effect of medical care to vary by illness

\footnotetext{
${ }^{25}$ Death is only observed once in the data because the estimation sample includes ages $19-64$, so it is not modeled as a possible health outcome. A death state would be a simple addition with alternative data sets.

${ }^{26}$ See Section 5.1 for a discussion of estimation and interpretation of unobserved permanent individual heterogeneity in this model.
} 
state entering the month.

I define a chronic illness to be any medical condition that never subsides (e.g., diabetes, asthma, AIDS) or, under normal conditions, has a permanent effect on an individual's health or medical care consumption (e.g., cancer, stroke, hypertension). ${ }^{27}$ Given the long-lasting effect of these ailments on health and/or medical care purchasing behavior, the occurrence of a chronic illness is modeled as a permanent, absorbing state. However, medical care can be used to control a chronic illness so that it has a lesser negative impact on an individual's general health status and acute illness probability. I model the probability that an individual is in a chronic illness state in month $t$ as a logistic function such that

$$
P\left(C_{t}=1\right)=\gamma_{t}^{1}= \begin{cases}\frac{\exp \left(\delta_{0}+\delta_{1} \mathbf{W}_{\mathbf{t}}+\delta_{2} \mathbf{H}_{\mathbf{t}}^{\mathbf{C}}+\delta_{3} \mathbf{M}_{\mathbf{t}-\mathbf{1}}+\delta_{4} \mathbf{N}_{\mathbf{t}}^{\mathbf{C}}+\mu_{2}^{k}\right)}{1+\exp \left(\delta_{0}+\delta_{1} \mathbf{W}_{\mathbf{t}}+\delta_{2} \mathbf{H}_{\mathbf{t}}^{\mathbf{C}}+\delta_{3} \mathbf{M}_{\mathbf{t}-\mathbf{1}}+\delta_{4} \mathbf{N}_{\mathbf{t}}^{\mathbf{C}}+\mu_{2}^{k}\right)} & \text { if } C_{t-1}=0 \\ 1 & \text { if } C_{t-1}=1\end{cases}
$$

where $\mathbf{H}_{\mathbf{t}}^{\mathbf{C}}=\left(\mathbb{1}_{H_{t}<2}, \mathbb{1}_{H_{t}<1}, A_{t-1}\right){ }^{28}$

At the end of each month $t$ an individual's general health status is updated, $H_{t+1}$, before transitioning to the next month $t+1$. Motivation for the inclusion of general health status comes from the household production approach of Grossman (1972), who describes both a

\footnotetext{
${ }^{27}$ This definition classifies several permanent physiological conditions as chronic illnesses that are not typically categorized as such, because the conditions are likely to impact an individual's future medical care consumption (e.g., amputations, menopause, organ and joint replacement).

${ }^{28}$ The MEPS data classifies illness by ICD-9-CM condition codes, which would allow for a more detailed characterization of illness to be integrated into the model. However, any attempt to more narrowly define illness states would require a significant level of subjectivity and would increase estimation time. For example, I could feasibly model the number of acute and chronic illnesses rather than using the dichotomous classification; however, this extension would require an assumption about whether or not a record of reported diarrhea and reported stomach-ache constitute one illness or two. Similarly, I could define different observed or unobserved classes of acute and chronic illness (Gilleskie 1998), but again, subjectivity is required in the classification of reported illnesses. Ultimately, empirical implementation of the model as specified reveals how well the three health measures (i.e., monthly transitions in general health status, acute illness state, and chronic illness contraction) and unobserved permanent individual heterogeneity explain medical care consumption behavior. The answer to this question is important given the models of moral hazard that consider only annual medical care consumption and one (or no) measure of health variation.
} 
consumption motive and a production motive for the utilization of medical care. In Grossman's model, an individual consumes medical care to rebuild an ever depreciating stock of health. The health stock produces health flows (e.g., healthy days), which directly increase utility and can be used to produce income or other consumption goods. The model presented in this research takes a similar approach by allowing general health status to enter the utility function and by allowing general health status to be influenced by past health and medical care consumption. I assume general health status has the following ordered structure

$$
\begin{gathered}
H_{t+1}^{*}=\psi_{0}+\psi_{1} \mathbf{W}_{\mathbf{t}}+\psi_{2} \mathbf{H}_{\mathbf{t}}^{\mathbf{H}}+\psi_{3} \mathbf{M}_{\mathbf{t}}+\psi_{4} \mathbf{N}_{\mathbf{t}}^{\mathbf{H}}+\mu_{3}^{k}+\zeta_{t+1} \\
\qquad \begin{array}{ll}
2 & \text { if } \kappa<H_{t+1}^{*} \\
1 & \text { if } 0<H_{t+1}^{*} \leq \kappa \\
0 & \text { if } H_{t+1}^{*} \leq 0
\end{array}
\end{gathered}
$$

where $H_{t+1}^{*}$ represents latent general health, $\mathbf{H}_{\mathbf{t}}^{\mathbf{H}}=\left(\mathbb{1}_{H_{t}<2}, \mathbb{1}_{H_{t}<1}, A_{t}, C_{t}\right)$, and $\kappa$ is a cutoff point to be estimated. Assuming $\zeta_{t+1}$ follows a logistic distribution, the (ordered logit) probability of transitioning to each general health level is

$$
\begin{aligned}
& P\left(H_{t+1}=2\right)=\eta_{t+1}^{2}=1-\Lambda\left(\kappa-\psi Z_{t}\right) \\
& P\left(H_{t+1}=1\right)=\eta_{t+1}^{1}=\Lambda\left(\kappa-\psi Z_{t}\right)-\Lambda\left(-\psi Z_{t}\right) \\
& P\left(H_{t+1}=0\right)=\eta_{t+1}^{0}=\Lambda\left(-\psi Z_{t}\right)
\end{aligned}
$$

where $\psi=\left(\psi_{0}, \ldots, \psi_{3}, \mu_{3}^{k}\right), Z_{t}=\left(\mathbf{W}_{\mathbf{t}}, \mathbf{H}_{\mathbf{t}}^{\mathbf{H}}, \mathbf{M}_{\mathbf{t}}, \mathbf{N}_{\mathbf{t}}^{\mathbf{H}}\right)$, and $\Lambda(\cdot)$ is the logistic function. In addition to its theoretical relevance, general health status plays an important role in this model because it gives purpose to medical care consumption when in a chronic illness state, which is important given that chronic illnesses never expire. Through interactions, this specification allows medical care consumption to alter the effect that a chronic illness state has on general health status. For example, according to this model, an individual with diabetes uses insulin 
to lessen the negative impact of the disease on his general level of health - not to cure the disease. The same could be said of open heart surgery, blood-pressure medication, or asthmatic inhalers.

\subsection{Utility Function and Budget Constraint}

Preferences for a medical care consumption bundle $\left(v_{t}=v, s_{t}=s, r_{t}=r\right)$ in month $t$ are described by the following contemporaneous utility function ${ }^{29}$

$$
\begin{aligned}
U\left(X_{t}, R, \mathbf{H}_{\mathbf{t}}^{\mathbf{U}}, d_{t}^{v s r}, \mu^{k}, \epsilon_{t}^{v s r}\right) & =\frac{X_{t}^{\omega_{0} R}}{\omega_{0} R}+\omega_{1} \mathbf{H}_{\mathbf{t}}^{\mathbf{U}}+\omega_{2} \mathbf{M}_{\mathbf{t}}^{\mathbf{U}}+\omega_{3} \mathbf{N}_{\mathbf{t}}^{\mathbf{U}}+\mu_{4 v}^{k}+\mu_{5 s}^{k}+\mu_{6 r}^{k}+\epsilon_{t}^{v s r} \\
& =\bar{U}\left(d_{t}^{v s r}\right)+\epsilon_{t}^{v s r}
\end{aligned}
$$

where $X_{i t}$ represents consumption of non-medical goods (determined by the budget constraint defined in Equation 3.8); $R=(1$, sex, race, age $) ; \mathbf{H}_{\mathbf{t}}^{\mathbf{U}}=\left(\mathbb{1}_{H_{t}<2}, \mathbb{1}_{H_{t}<1}, A_{t}, C_{t}\right)$; $\mathbf{M}_{\mathbf{t}}^{\mathbf{U}}=\left(v_{t}, s_{t}, r_{t}, v_{t}^{2}, s_{t}^{2}\right) ; \mathbf{N}_{\mathbf{t}}^{\mathbf{U}}$ contains interactions of the variables in $\left(\mathbf{H}_{\mathbf{t}}^{\mathbf{U}}, \mathbf{M}_{\mathbf{t}}^{\mathbf{U}}, \mathbf{W}_{\mathbf{t}}\right) ;$ the $\mu^{k}$ parameters capture unobserved permanent heterogeneity for an individual of type $k$; and $\epsilon_{i t}^{v s r}$ is the unobserved utility received from $v$ doctor visits, $s$ hospital days, and consuming prescription drugs $(r=1)$ or not $(r=0) .{ }^{30}$

The monthly budget constraint is

$$
X_{t}=Y_{t}-P_{j t}-O_{t}\left(v_{t}, s_{t}, r_{t}, p_{t}^{v}, p_{t}^{s}, p_{t}^{r}, A D E_{t}, A H E_{t}, I_{y}^{j}\right)
$$

where $Y_{i t}$ is monthly income; $P_{i j t}$ is the month $t$ premium paid out-of-pocket for plan $j ; O_{t}(\cdot)$

\footnotetext{
${ }^{29}$ This utility function is representative when $X_{t}$ is greater than or equal to zero. If medical care expenditure becomes so great that $X_{t}$ is negative, then the first term $\frac{X_{t}^{\omega_{0} R}}{\omega_{0} R}$ is replaced by $\omega_{40} * X_{t}$ to capture the (dis)utility of negative non-medical good consumption.

${ }^{30}$ I allow the effect of unobserved permanent individual heterogeneity in the utility function to vary by polynomials in consumption. Thus, unobserved preference for doctor visits is captured by $\mu_{4 v}=v \mu_{4}^{a}+v^{2} \mu_{4}^{b}$; hospital days by $\mu_{5 s}=s \mu_{5}^{a}+s^{2} \mu_{5}^{b}$; and prescription drugs by $\mu_{6 r}$.
} 
is the out-of-pocket expenditure on medical care in month $t ; p_{i t}^{v}, p_{i t}^{s}$, and $p_{i t}^{r}$ represent the total price of a doctor visit, a hospital day, and prescription drugs, respectively; and $A D E_{i t}$ and $A H E_{i t}$ represent accumulated out-of-pocket medical care expenditure for doctor visits and hospital days entering month $t$, respectively. ${ }^{31}$ This structure assumes that an individual consumes all income by the end of each month, as monthly saving decisions are not observed in the data. ${ }^{32}$

Having specified the contemporaneous utility function, budget constraint, and all transitions between uncertain illness states and general health status, I denote the set of information known by an individual at the time of a medical care consumption decision, or his state, as $\Psi_{t}=\left(W_{t}, H_{t}, A_{t}, C_{t}, A D E_{t}, A H E_{t}, I_{y}^{j}, \mu^{k}, \epsilon_{t}^{v s r}\right)$. It remains to describe what the model assumes about an individual's knowledge of medical care prices and out-of-pocket expenditure; then, the optimization problem can be fully expressed.

\subsection{Medical Care Prices and Expenditure}

Two characteristics of the medical care marketplace make within-year medical care expenditure an important economic construct. First, most individuals do not pay the total price of medical care because of a cost-sharing arrangement with their health insurance provider. ${ }^{33}$ Rather, an individual pays a dollar amount out-of-pocket that is determined by the total price of medical care, insurance plan characteristics, and accumulated medical care expenditure

\footnotetext{
${ }^{31}$ Because an individual faces a binary decision on whether or not to consume any prescription drugs, $p_{i t}^{r}$ is a total monthly expenditure on prescription drugs rather than the unit price per prescription.

${ }^{32}$ French and Jones (2011) examine the effects of health insurance and self-insurance (i.e., savings) on retirement behavior. The authors explain that omitting savings from an individual's dynamic problem ignores the ability to smooth consumption through savings, which can potentially overstate the value of insurance. In simulation, they find that omitting savings from the model does increase the value of insurance, but (retirement) decision making is unchanged in the no-savings model.

${ }^{33}$ Medical care can be thought of as having two prices, a list price and a transaction price. The list price is generally printed on a customer's bill and can be thought of as the theoretical market price for care. The transaction price, which is the sum of the insurer's and insured's payments, is typically lower than the list price as insurance companies negotiate for reduced rates from certain medical care providers. Because the list price is rarely paid in practice, the total price in this model refers to the total transaction amount for a unit of medical care, which I observe in the data.
} 
during the coverage year. For example: an individual with a $\$ 300$ deductible, $10 \%$ coinsurance rate, and $\$ 0$ of accumulated expenditure who is charged $\$ 100$ for a doctor visit pays the full $\$ 100$ out-of-pocket. However, if the same individual were to have accumulated $\$ 250$ in medical care expenditure prior to the visit, then he would pay only $\$ 55$ out-of-pocket for the visit ( $\$ 50$ pre-deductible $+\$ 5[=0.1 *(\$ 100-\$ 50)]$ post-deductible). An individual with health insurance characterized by this cost-sharing structure (i.e., a deductible with a co-insurance rate) faces a non-linear budget constraint. The out-of-pocket expenditure function, $O_{t}(\cdot)$, is constructed so that the budget constraint in Equation 3.8 contains these nonlinearities. Precise calculations of out-of-pocket expenditure and accumulated out-of-pocket expenditure are detailed in Appendix C.

A second characteristic of the medical care market is that individuals are typically uncertain of the total price of medical care prior to consumption. The lack of menu prices, uncertainty of diagnosis prior to a visit, and wide price variation in local medical care markets contribute to price uncertainty. ${ }^{34}$ Despite the evidence, surprisingly few models of medical care demand allow for this uncertainty. To address this reality, I assume that an individual does not observe total medical care prices prior to making a medical care decision in each month. Rather, an individual knows the conditional distributions from which doctor visit prices, hospital day prices, and prescription drug prices are drawn. An individual makes medical care decisions by integrating over the three conditional price distributions, which are estimated from the data. ${ }^{35}$

The total price distributions are defined as $F^{v}\left(p_{t}^{v} \mid \Phi_{t} ; \lambda^{v}\right), F^{s}\left(p_{t}^{s} \mid \Phi_{t} ; \lambda^{s}\right)$, and $F^{r}\left(p_{t}^{r} \mid \Phi_{t} ; \lambda^{r}\right)$, where $\Phi_{t}=\left(W_{t}, H_{t}, A_{t}, C_{t}, H M O_{j}, P P O_{j}, F F S_{j}, \mu^{k}\right)$ is a vector of variables that explain

\footnotetext{
${ }^{34}$ In May 2013, the Centers for Medicare and Medicaid Services (CMS) released data showing wide variation in medical care prices in local medical care markets. Such variation makes it difficult for an individual to know medical care prices prior to consumption. Recent articles in Time Magazine and The New York Times have also highlighted the issue of price uncertainty in medical care markets.

${ }^{35}$ An equilibrium model of the medical care market could conceivably allow for price determination in solution. Such a model, to be realistic, would have to include as players individuals, providers, hospitals, insurance companies, employers, and the government since interactions between all of these entities determine prices in the market.
} 
variation in the distributions and $\left(\lambda^{v}, \lambda^{s}, \lambda^{r}\right)$ are parameters to be estimated. The variables $H M O_{j}, P P O_{j}$, and $F F S_{j}$ are indicators of the plan's coverage type. Coverage type is included to capture the negotiation for lower rates by insurance providers who contract with a network of physicians. ${ }^{36}$ An indicator of MSA level is included in $W_{t}$ to capture urban area variation in prices. In addition to differences attributed to supply side variation, these distributions depend on individual observed illness states and general health status. Finally, these medical care price shocks are likely to be correlated with unobserved illness and health shocks. An individual who receives an exceptionally bad illness shock (e.g., cancer) is also likely to experience a price distribution that is shifted upward or has fatter tails. For this reason, the three medical care price shocks are likely to be correlated with one another as well. I allow the permanent unobservables that influence preferences, illness states, and general health outcomes to also influence the price distributions. Currently, the model does not allow for time-varying unobserved heterogeneity.

\subsection{The Optimization Problem}

An individual's objective is to maximize his expected discounted future utility by selecting the optimal sequence of medical care bundles, $d_{t}^{v s r}$, for $t=1, \ldots, T$ and insurance plans, $I_{y}^{j}$, for $y=1, \ldots Y$ conditional on his state variables in $\Psi_{t}$. I describe an individual's dynamic optimization problem in two stages, as insurance decisions are made at the beginning of a year and medical care is chosen repeatedly over the course of a year.

\subsubsection{The Optimal Monthly Decision Rule}

Let $V_{v s r}^{a c h}\left({ }_{t}\right)$ be the month $t$ value of expected discounted future utility for medical care decision $d_{t}^{v s r}$, illness state $\left(A_{t}=a, C_{t}=c\right)$, and general health status $\left(H_{t}=h\right)$. Using Bellman's Equation (Bellman 1957), this value is constructed as the sum of contemporaneous

\footnotetext{
${ }^{36}$ The model does not differentiate between in-network and out-of-network medical care consumption. All medical care is assumed to be in-network. Insurance cost-sharing characteristics are specific to in-network consumption.
} 
utility and the expected discounted future utility yielded by the alternative. Conditional on unobserved heterogeneity type $k$ (where $\mu^{k}=\left\{\mu_{1}^{k}, \ldots, \mu_{14}^{k}\right\}$ ), insurance plan $j$, and medical care prices $\left(p_{t}^{v}, p_{t}^{s}, p_{t}^{r}\right)$, the alternative-specific value function can be written, for $t<T$

$$
\begin{aligned}
& V_{v s r}^{a c h}\left(\Psi_{t}, \epsilon_{t}^{v s r} \mid \mu^{k}, I_{y}^{j}, p_{t}^{v}, p_{t}^{s}, p_{t}^{r}\right)=\bar{U}\left(d_{t}^{v s r}\right)+\epsilon_{t}^{v s r} \\
& \quad+\beta \sum_{h^{\prime}=0}^{2}\left[\eta_{t+1}^{h^{\prime}}\left(\Psi_{t}, d_{t}^{v s r}\right) \sum_{a^{\prime}=0}^{1} \pi_{t+1}^{a^{\prime}}\left(h^{\prime}, \Psi_{t}, d_{t}^{v s r}\right) \sum_{c^{\prime}=0}^{1} \gamma_{t+1}^{c^{\prime}}\left(h^{\prime}, \Psi_{t}, d_{t}^{v s r}\right)\left[V^{a^{\prime} c^{\prime} h^{\prime}}\left(\Psi_{t+1} \mid \mu^{k}, I_{y}^{j}\right)\right]\right],
\end{aligned}
$$

and for $t=T$

$$
V_{v s r}^{a c h}\left(\Psi_{t}, \epsilon_{t}^{v s r} \mid \mu^{k}, I_{y}^{j}, p_{t}^{v}, p_{t}^{s}, p_{t}^{r}\right)=\bar{U}\left(d_{t}^{v s r}\right)+\epsilon_{t}^{v s r}+\beta \sum_{h^{\prime}=0}^{2}\left[\eta_{t+1}^{h^{\prime}}\left(\Psi_{t}, d_{t}^{v s r}\right)\left[Q_{y+1}\left(\Psi_{0}, h^{\prime}\right)\right]\right]
$$

where $\bar{U}\left(d_{t}^{v s r}\right)$ is the deterministic part of Equation 3.7, $\beta$ is the discount factor, and $Q_{y+1}\left(\Psi_{0}, h^{\prime}\right)$ is the value of expected discounted future utility in month $t=0$ of year $y+1$. Maximal expected utility, in illness state $\left(A_{t+1}=a^{\prime}, C_{t+1}=c^{\prime}\right)$ with general health status $\left(H_{t+1}=h^{\prime}\right)$, in month $t+1$ is

$$
V^{a^{\prime} c^{\prime} h^{\prime}}\left(\Psi_{t+1} \mid \mu^{k}, I_{y}^{j}\right)=E_{t}\left[\max _{v s r} V_{v s r}^{a^{\prime} c^{\prime} h^{\prime}}\left(\Psi_{t+1}, \epsilon_{t+1}^{v s r} \mid \mu^{k}, I_{y}^{j}\right)\right]
$$

The expectation operator is subscripted by $t$ because an individual must form this expectation prior to learning month $t+1$ medical care preference shocks, $\epsilon_{t+1}^{v s r}$.

The value function in Equation 3.9 is written conditional on realized medical care prices. However, it is assumed that an individual does not know the prices of the three types of medical care prior to consumption; rather, he knows the conditional distributions from which these prices are drawn. Solution to the optimization problem requires integration over these 
price distributions. The value function, unconditional on prices, is

$$
V_{v s r}^{a c h}\left(\Psi_{t}, \epsilon_{t}^{v s r} \mid \mu^{k}, I_{y}^{j}\right)=\int_{\mathbb{R}_{+}^{3}} f^{*}\left(p_{t}^{v}, p_{t}^{s}, p_{t}^{r}\right) V_{v s r}^{a c h}\left(\Psi_{t}, \epsilon_{t}^{v s r} \mid \mu^{k}, I_{y}^{j}, p_{t}^{v}, p_{t}^{s}, p_{t}^{r}\right) d p_{t}^{v} d p_{t}^{s} d p_{t}^{r}
$$

where $f^{*}\left(p_{t}^{v}, p_{t}^{s}, p_{t}^{r}\right)=f^{v}\left(p_{t}^{v}\right) * f^{s}\left(p_{t}^{s}\right) * f^{r}\left(p_{t}^{r}\right)$ and $f^{v}(\cdot), f^{s}(\cdot)$, and $f^{r}(\cdot)$ are the conditional density functions from which $p_{t}^{v}, p_{t}^{s}$, and $p_{t}^{r}$ are drawn. ${ }^{37}$

Conditional on the prior insurance decision and unobserved heterogeneity, a utility maximizing individual selects each medical care consumption bundle with probability

$$
P\left(d_{t}^{v s r}=1\right)=P\left[V_{v s r}^{a c h}\left(\Psi_{t}, \epsilon_{t}^{v s r} \mid \mu^{k}, I_{y}^{j}\right) \geq V_{v^{\prime} s^{\prime} r^{\prime}}^{a c h}\left(\Psi_{t}, \epsilon_{t}^{v^{\prime} s^{\prime} r^{\prime}} \mid \mu^{k}, I_{y}^{j}\right) \quad \forall v^{\prime} s^{\prime} r^{\prime}\right]
$$

\subsubsection{The Optimal Annual Decision Rule}

The problem can be solved backwards to recover the time $t=0$, year $y$ value function conditional on any chosen health insurance alternative $j \in J_{y}^{i}$. That is,

$$
V\left(\Psi_{0}, H_{1}=h \mid \mu^{k}, I_{y}^{j}\right)=\sum_{a=0}^{1} \pi_{1}^{a}\left(\Psi_{0}, H_{1}\right) \sum_{c=0}^{1} \gamma_{1}^{c}\left(\Psi_{0}, H_{1}\right)\left[V^{a c h}\left(\Psi_{1} \mid \mu^{k}, I_{y}^{j}\right)\right]
$$

Stated explicitly, Equation 3.14 represents the discounted value of optimal future behavior calculated at the beginning of year $y$ unconditional on the first month acute and chronic illness state but conditional on general health status entering the year and insurance plan $j$ (i.e., the expected discounted future value of plan $j$ ). ${ }^{38}$ This value does not completely determine the optimal insurance alternative, as an individual may have preferences for unobserved insurance characteristics. ${ }^{39}$ Therefore, I allow further variation through an additive error term such that

\footnotetext{
${ }^{37}$ Conditional on $\mu^{k}$ (unobserved permanent individual heterogeneity) these distributions are independent; however, their dependence on $\mu^{k}$ allows some correlation.

${ }^{38}$ Notice that general health status in month $1, H_{1}$, is already known at this time because it was learned during the last month of the prior year.

${ }^{39}$ Unobserved characteristics could be defined coverage restrictions, such as a preexisting condition clause or referral requirement to see a specialist, which are not modeled; they could also be undefined characteristics
} 
the expected discounted future value of plan $j$ is

$$
Q_{y}^{j}\left(\Psi_{0}, H_{1}, \phi_{y}^{j} \mid \mu^{k}\right)=V\left(\Psi_{0}, H_{1} \mid \mu^{k}, I_{y}^{j}\right)+\phi_{y}^{j}
$$

A utility maximizing individual selects each insurance plan with the probability 40

$$
P\left(I_{y}^{j}=1\right)=P\left[Q_{y}^{j}\left(\Psi_{0}, H_{1}, \phi_{y}^{j} \mid \mu^{k}\right) \geq Q_{y}^{j^{\prime}}\left(\Psi_{0}, H_{1}, \phi_{y}^{j^{\prime}} \mid \mu^{k}\right) \quad \forall j^{\prime}\right]
$$

This optimization problem is consistent with our theoretical understanding of insurance benefits. Health insurance is valuable because it provides risk protection, allows for higher non-medical consumption when ill, and yields health benefits if additional medical care is consumed during a coverage year. Further, the model explicitly captures the non-linear relationship between a plan's expected value at the beginning of a year, its cost-sharing characteristics, and an individual's uncertainty about his future health, medical care prices, and medical care demand.

that are unlikely to alter the value of a plan during the year but influence individual decisions, such as the plan's order on the application file or brand name. Choice inertia, or the tendency of individuals to simply select the same health insurance plan that they had in the previous year, is another unobserved factor that may influence an individual's observed plan. Handel (2013) finds evidence of substantial inertia in the dynamic insurance decisions of employees at one large American firm.

\footnotetext{
${ }^{40}$ In the optimization problem, an individual has knowledge of $\Psi_{0}$ at the time of an insurance decision, where $\Psi_{0}$ contains the chronic illness state learned in the last month of the previous insurance year. In the data, it cannot always be determined whether a chronic illness present in first month of the insurance year (which is often the first month of the survey period) began in the first month or a previous month. Therefore, in order to allow an individual to make health insurance decisions with knowledge of existing chronic illnesses, I assume (in estimation) that he learns his first month chronic illness state prior to his health insurance decision in the first year of optimization.
} 


\section{DATA}

My empirical analysis uses data from the 1996-1999 cohorts of the Medical Expenditure Panel Survey (MEPS). ${ }^{41}$ MEPS contains detailed health, medical care expenditure, health insurance, and demographic information for a nationally representative sample of families and individuals in the United States. New participants are added annually (beginning in 1996 through the present day), drawn randomly from the previous year's National Health Interview Survey sample. Individuals in each cohort are interviewed 5 times over the 2 years that follow January 1 st of their cohort year.

The MEPS has two features that make it particularly well suited for the purposes of this research. First, detailed employer level insurance information that can be linked to the individual file was collected for the 1996-1999 cohorts. Data collectors used information gathered in the first interview to contact current main employers, from which they obtained premium and cost-sharing characteristics for all plans offered to the employee. This data feature, which is unique in national survey data, enables me to model a health insurance decision from the full set of available alternatives for individuals with participating employers. However, roughly $50 \%$ of individuals participating in MEPS are without insurance information in this link file due to employee and/or employer refusal to reveal information. ${ }^{42}$ Also, while individuals are

\footnotetext{
${ }^{41}$ The data are collected and maintained by the Agency for Healthcare Research and Quality (AHRQ). All data used in estimation are publicly available, with the exception of the individual insurance plan information. These restricted files may only be accessed through a Census Bureau Research Data Center (RDC).

${ }^{42}$ There was one significant change to the collection process that took place after 1996. The 1996 MEPS asked each employer specific questions regarding their participating employee. This method caused many employees to refuse to provide their employers information as employees wished to remain anonymous. The method was also inconvenient for employers because it was much more difficult to provide information about a particular employee than employees in general. (Legalities also made employers weary of providing employee-specific information.) Therefore, in 1997 the collection process was altered such that employers were asked about their general insurance offerings, but not employee specific offerings. AHRQ then used a matching procedure to identify which offered plan was reportedly chosen by employees.
} 
interviewed over the course of two years, few employers agree to provide health insurance plan information at the beginning of each year. Therefore, analysis concentrates on one health insurance decision and the medical care decisions in the year that follows for each individual. Second, unlike claims data, the MEPS allows participants to report illness episodes even when they choose not to consume medical care. This data feature allows endogenous illness transitions to be modeled.

A number of important assumptions are required to prepare the data for estimation. For example, each illness, which is defined in the data by an ICD-9-CM medical code, must be interpreted as an acute or chronic illness. Also, medical care consumption dates and partially observable illness dates must be used to determine the starting and ending month of illnesses reported five times over the course of 2 years. I also face the challenge that at least one of the 12 insurance cost-sharing features is missing in $47 \%$ of the 5284 plans observed in the data, so imputations must be made. The magnitude of these complications, and others, and the assumptions required to overcome them are discussed at length in Appendix B.

\subsection{Determination of the Sample}

The sample used in estimation is taken from the nationally representative sample of single and childless individuals included in the 1996-1999 cohorts of the MEPS survey. (See Table 4.1 for sample size by cohort year and inclusion criteria.) I focus on employed individuals between the ages of 19 and 64 whose employers sponsor health insurance coverage. ${ }^{43}$ I exclude the unemployed and those employed without an insurance offer because only general insurance information was gathered for these individuals (e.g., coverage status, coverage source, etc.). Employed individuals who receive an insurance offer but choose to be uninsured are included in the estimation sample. These omissions are representative of the sample restrictions

\footnotetext{
${ }^{43}$ I study individuals over 18 years old to avoid the unique decision-making process of an adolescent with possible access to his parents health insurance plans. I also exclude full time students under the age of 24 because 1996-1999 federal law allowed these individuals to stay on their parent's insurance plan. Individuals under 64 are targeted because they do not yet have access to coverage through Medicare.
} 
found in similar work. ${ }^{44}$

Sample inclusion also requires that the ESHI plans that are offered to an individual are observed in the link file described above. The information contained in the link file is necessary to model an individual's insurance decisions. Individuals must also participate in all interviews during the insurance year. The final restriction limits individuals in the sample to one of two types: (1) individuals taking up ESHI, holding it for an entire year, and holding no outside coverage; or (2) individuals remaining completely uninsured all year. I do not model insurance switching during an insurance year and cannot observe privately purchased plan characteristics.

Table 4.1: Sample Inclusion Criteria

\begin{tabular}{lrrrrr}
\hline & 1996 & 1997 & 1998 & 1999 & Total \\
\hline 1996-1999 MEPS Household Component & 22601 & 13683 & 11137 & 14178 & 61599 \\
and single, childless, 19-64 yrs old & 4406 & 2534 & 2169 & 2589 & 11698 \\
and employed in first interview period with offer & 1821 & 923 & 987 & 1128 & 4859 \\
and matches to link file & 749 & 516 & 159 & 688 & 2112 \\
and no missing interviews & 693 & 472 & 139 & 636 & 1940 \\
and stable insurance status & 455 & 290 & 98 & 389 & 1232 \\
\hline
\end{tabular}

$\dagger$ There is a disproportionate drop in link file matches in 1998 because AHRQ only attempted to contact employers for $25 \%$ of survey participants who reported being offered health insurance coverage. In all other years, AHRQ attempted to contact employers for all of these individuals.

The final estimation sample contains 1232 individuals (or 14784 person-month observations). Table A.1, which can be found in Appendix A, compares the 4859 individuals remaining at line 3 above (which is a nationally representative sample of single, childless, 19-64 year olds, who are employed and offered health insurance from their employer) and those in the estimation sample. The table reveals few differences between the estimation sample and a nationally representative sample of this demographic. The estimation sample is slightly

\footnotetext{
${ }^{44}$ Cardon and Hendel (2001) limit their sample to single, childless, employed individuals who are between the ages of 18 and 64. However, these authors include individuals who are not offered health insurance by their employer. Einav et al. (2013), Kowalski (2013), and Bajari et al. (2013) estimate their models using a sample of individuals employed by one firm. None of the papers include uninsured individuals in the analysis. Einav et al. (2013) model the decisions of families. Kowalski (2013) models the behavior of individual employees, but allows individuals to be in a family of three or fewer people. Bajari et al. (2013) limits analysis to those holding single health insurance coverage.
} 
older, a little wealthier, and is comprised of a larger proportion of females. These differences contribute to higher medical care expenditure in the estimation sample. The estimation sample is also comprised of more federal employees, which is expected, as no federal employees are excluded due to employer non-response.

\subsection{Sample Statistics}

The following tables summarize the mean and dispersion of key variables used in estimation. Table 4.2 compares insured and uninsured individuals in the estimation sample. ${ }^{45}$ The insured are older, more educated, and wealthier. They are also more likely to be white and female. The insured are more likely to enter the insurance year with a chronic illness, are more likely to get an acute illness at some point during the insurance year, and have more months where some acute illness is experienced. The insured also consume more units and greater values of doctor and prescription drug care. ${ }^{46}$ The percentage of the population that consumes at least one hospital day during the year (25\% of insured and $17 \%$ of uninsured individuals) seems large, but includes emergency room visits as well as outpatient and inpatient visits. To improve estimation time, I limit the maximum number of doctor visits and hospital days in a month ( $V$ and $S$ from Equation 3.2) to 9 and 5, respectively. ${ }^{47}$ The insured face lower (total) prices for doctor visits and hospital days and higher prices for prescription drugs.

\footnotetext{
${ }^{45}$ Most variables are self-explanatory. Income is calculated as the sum of post-tax income, sale earnings, and tax refund. General health status is self-reported, taken from the response to the question "In general, compared to other people of your age, would you say that your health is excellent, good, fair, poor, or very poor?" Roughly $6 \%$ of the estimation sample reports poor or very poor health, so the lowest three health categories (fair, poor, and very poor) are combined to form the poor general health status category seen in the table and used in estimation. Medical care prices are only observed when medical care is consumed. For more detail on medical care prices, medical care consumption, and illness occurrence see Appendix B.

${ }^{46}$ While the uninsured are less likely to have at least one hospital day, the average number of hospital days for the uninsured is greater than that of the insured. This is likely due to emergency room usage among the uninsured.

${ }^{47}$ Of the 14,784 person-month observations in the data, the number of doctor visits exceeds the maximum of 9 only 33 times and the number of hospital days exceeds the maximum of 5 only 30 times. In these instances, the number of visits/days is set to the maximum and the average price paid for a unit of medical care is adjusted accordingly. For example, if an individual visits the doctor 12 times in a month with an average price of $\$ 100$ then the data are adjusted so that he visits the doctor 9 times with an average price of $\$ 133$.
} 
Table 4.2: Sample Statistics by Insurance Status

\begin{tabular}{|c|c|c|c|c|}
\hline & \multicolumn{2}{|c|}{ Insured } & \multicolumn{2}{|c|}{ Uninsured } \\
\hline & mean & s.d. & mean & s.d. \\
\hline \multicolumn{5}{|l|}{ Demographics (time invariant) } \\
\hline age & 40.04 & 11.62 & 34.73 & 11.44 \\
\hline education (highest grade completed) & 13.73 & 2.43 & 12.46 & 2.49 \\
\hline income (in 1996 dollars) & 35198.52 & 22120.24 & 19011.55 & 13144.27 \\
\hline male & 0.48 & $*$ & 0.52 & $*$ \\
\hline lives in a MSA & 0.82 & $*$ & 0.83 & * \\
\hline Hispanic & 0.11 & $*$ & 0.21 & * \\
\hline black & 0.14 & $*$ & 0.19 & * \\
\hline federal employee & 0.09 & * & 0.09 & * \\
\hline \multicolumn{5}{|l|}{ Health and Illness (time varying) } \\
\hline probability of excellent health status in any month & 0.31 & $*$ & 0.32 & * \\
\hline probability of good health status in any month & 0.36 & $*$ & 0.34 & * \\
\hline probability of poor health status in any month & 0.33 & $*$ & 0.34 & * \\
\hline entered year with chronic illness & 0.39 & * & 0.25 & * \\
\hline chronic illness by years end & 0.50 & $*$ & 0.31 & * \\
\hline at least one acute illness during sample year & 0.81 & $*$ & 0.69 & * \\
\hline total months with acute illness & 4.62 & 4.52 & 2.35 & 3.21 \\
\hline \multicolumn{5}{|l|}{ Medical Care Prices (time varying) } \\
\hline transaction price for a doctor visit & 89.19 & 159.22 & 112.43 & 160.52 \\
\hline transaction price for a hospital day & 823.31 & 1357.76 & 878.72 & 2127.58 \\
\hline transaction price for a Rx month & 75.22 & 114.94 & 49.77 & 54.43 \\
\hline \multicolumn{5}{|l|}{ Medical Care Consumption (time varying) } \\
\hline at least one doctor visit in sample year & 0.74 & $*$ & 0.50 & * \\
\hline total doctor visits in sample year & 5.22 & 9.35 & 2.67 & 5.64 \\
\hline at least one hospital day in sample year & 0.25 & $*$ & 0.17 & * \\
\hline total hospital days in sample year & 0.75 & 2.41 & 0.99 & 4.51 \\
\hline at least one $\mathrm{Rx}$ month in sample year & 0.67 & $*$ & 0.45 & * \\
\hline total Rx months in sample year & 4.69 & 5.00 & 2.22 & 3.91 \\
\hline consumed any preventative care & 0.20 & $*$ & 0.19 & * \\
\hline probability of consumption in any month & 0.62 & * & 0.45 & * \\
\hline probability of consumption in well month & 0.13 & $*$ & 0.05 & $*$ \\
\hline annual value of doctor visits & 454.39 & 951.61 & 289.67 & 759.21 \\
\hline annual value of hospital days ${ }^{\dagger}$ & 660.28 & 2706.30 & 1108.39 & 6320.84 \\
\hline annual value of $\mathrm{Rx}$ consumption & 352.80 & 819.20 & 110.57 & 272.71 \\
\hline \multicolumn{5}{|l|}{ Other } \\
\hline number of offered plans & 4.41 & 5.99 & 3.05 & 4.46 \\
\hline \multicolumn{5}{|l|}{ Sample } \\
\hline individuals & 1119 & & 113 & \\
\hline person-month observations & 13428 & & 1356 & \\
\hline
\end{tabular}

† One uninsured individual had hospital expenditures totaling $\$ 52,032.16$. Removing this outlier lowers the mean to $\$ 647.93$ for the uninsured. 
Table 4.3: Insurance Plan Summary

\begin{tabular}{|c|c|c|c|c|c|c|}
\hline & \multicolumn{3}{|c|}{ Held Plans } & \multicolumn{3}{|c|}{ Rejected Plans } \\
\hline & plans & mean & s.d. & plans & mean & s.d. \\
\hline \multicolumn{7}{|l|}{ Premium } \\
\hline total premium & 1119 & 2057.19 & 819.09 & 4165 & 2207.13 & 715.25 \\
\hline out-of-pocket premium & 1119 & 343.83 & 540.31 & 4165 & 519.46 & 610.28 \\
\hline \multicolumn{7}{|l|}{ Deductible ${ }^{\dagger}$} \\
\hline defined by total expenditure & 397 & 283.59 & 272.99 & 772 & 293.25 & 348.26 \\
\hline defined by doctor expenditure only & 59 & 191.38 & 97.04 & 655 & 215.72 & 50.65 \\
\hline defined by hospital expenditure only & 28 & 252.63 & 218.10 & 62 & 150.58 & 41.24 \\
\hline plan has no deductible & 648 & $*$ & $*$ & 2684 & $*$ & * \\
\hline \multicolumn{7}{|l|}{ Stop loss } \\
\hline stop loss & 729 & 1512.82 & 1077.97 & 2775 & 1689.06 & 1077.63 \\
\hline plan has no stop loss & 390 & $*$ & $*$ & 1390 & $*$ & $*$ \\
\hline \multicolumn{7}{|l|}{ Hospital ${ }^{\ddagger}$} \\
\hline co-insurance rate & 417 & 17.02 & 9.91 & 797 & 15.44 & 8.01 \\
\hline co-pay level (per stay) & 199 & 258.46 & 346.70 & 768 & 159.41 & 194.45 \\
\hline co-pay level (per day) & 85 & 52.61 & 60.25 & 142 & 58.70 & 85.24 \\
\hline free care past the deductible & 435 & $*$ & * & 2577 & $*$ & * \\
\hline \multicolumn{7}{|l|}{ Doctor } \\
\hline co-insurance rate & 208 & 18.91 & 8.69 & 755 & 12.43 & 7.83 \\
\hline co-pay level & 858 & 10.19 & 4.53 & 3110 & 8.41 & 4.25 \\
\hline free care past the deductible & 53 & $*$ & $*$ & 300 & $*$ & * \\
\hline \multicolumn{7}{|l|}{ Network Type } \\
\hline HMO & 1119 & 0.42 & $*$ & 4165 & 0.51 & $*$ \\
\hline FFS & 1119 & 0.47 & $*$ & 4165 & 0.44 & $*$ \\
\hline PPO & 1119 & 0.11 & $*$ & 4165 & 0.06 & $*$ \\
\hline
\end{tabular}


The large variance in prices is due to the broad classification of medical care consumption types. High priced procedures (e.g., outpatient hospital surgery) and low priced procedures (e.g., emergency room visit for a sprained ankle) contribute to the same price distribution. An individual is considered to have consumed any preventative care if during the sample period they consume any form of medical care in a month in which they have no acute illness or chronic illness.

Differences between the chosen and rejected plans can be observed in Table 4.3. The table suggests that individuals have a general preference for lower premium and therefore less generous (in terms of cost-sharing features) plans. Compared to the average rejected plan, held plans are more likely to have a deductible, less likely to have a stop loss, and set higher thresholds when the plan has a deductible or stop loss. Held plans also feature higher co-insurance rates and co-pay levels for both doctor and hospital care, with the exception of hospital per day co-pay.

Though it cannot be taken directly from these tables, extracting insurer profit and mark-up information from the data is straightforward. Given total annual expenditure on doctor, hospital, and prescription drug services, insurers loose money on $16 \%$ of the observed contracts. The average loss is roughly $\$ 3800$. Conversely, insurers profit on the remaining $84 \%$ of observed contracts, with a mean profit of $\$ 1638$. These statistics suggest an average mark-up of $\$ 788$, or $38 \%$ above the average premium of $\$ 2057$. (Note: these figures assume that expenditure is limited to the three types of medical care consumption that is modeled. Because some additional types of consumption are covered, this is an upper bound on the markup.)

\subsection{Prescription Drugs}

Several assumptions are required to fit the prescription drug data available in the MEPS to the within-year decision-making model presented in Section 3. First, the employer questionnaire used to gather insurance information asks whether each plan "covers" outpatient prescriptions (99\% of all plans in the sample do), but does not ask the co-insurance or co-pay 
level, whether there is a separate deductible for prescription drugs, or whether cumulative deductibles and stop losses apply to prescription drugs expenditure. Therefore, I assume that HMO, PPO, and FFS plans have a fixed prescription drug co-insurance rate of $13 \%, 17 \%$, and $19 \%$ respectively. These rates are consistent with the average rates in the 1996 MEPS Abstraction file. ${ }^{48}$ I also assume that prescription drug expenditure is completely unrelated to a plan's deductible and stop loss. ${ }^{49}$ This assumption is also informed by the 1996 MEPS Abstraction file, which finds that prescription drug expenditure had no relation to a deductible for $77 \%$ of held plans and had no relation to the stop loss for $44 \%$. According to the Kaiser Family Foundation's Employer Health Benefits Annual Survey, by 2000 prescription drug expenditure had no relation to the stop loss in over $75 \%$ of ESHI plans.

Second, I assume that an individual decides whether or not to consume any prescription drugs each month, not the number of prescriptions to fill. In addition to making estimation more tractable, this assumption acknowledges the doctors role in the prescription drug decision. Often times multiple drugs are prescribed such that consuming two, as opposed to one, prescription drugs in no way reflects a marginal decision by an individual and is unlikely to further improve wellness. Also, the survey collects the number of refills of each prescription in each interview period, but provides an exact date only the first time a prescription is purchased. Furthermore, while the exact medication name and quantity are provided, there is no record of whether or not an individual actually consumed the medicine. Therefore, I assume that all patients are prescribed the average dosage for a medication. ${ }^{50}$ I then use this dosage,

\footnotetext{
${ }^{48}$ Co-pays are a much more popular form of cost-sharing for prescription drugs than co-insurance rates $(68 \%$ vs. $32 \%$ in 1996). However, co-pays make the number and timing of refills a relevant factor in analysis, which I would like to abstract from. Further, $80 \%$ of all ESHI plans feature multi-tier prescription drug coverage in the form of co-pays by 2000 (Kaiser EHBAS). Thus, correctly implementing co-pays for prescription drug coverage would require both a quantity and quality decision by individuals.

${ }^{49}$ Unrelated means that the insurer and insured share the total cost of prescription drugs from the first day of an insurance year to the last, irrespective of accumulated expenditure. Also, out-of-pocket prescription drug expenditure does not contribute to the accumulated expenditure relevant for the cumulative deductible or stop loss.

${ }^{50}$ I use "Mosby's Pharmacology in Nursing," to determine the average dosage of each medication found in the data. My imputations were then double checked by a doctor. More details can be learned in Appendix B.
} 
along with the date the prescription was first filled, the number of refills, and the quantity (which is usually number and strength of pills) of the drug in the prescription, to construct a starting date and ending date for each drug consumed. I then smooth payments for the drug over the course of the consumption period, because the model does not distinguish between the payment for and consumption of prescription drugs. 


\section{EMPIRICAL IMPLEMENTATION}

For each individual surveyed in the 1996-1999 MEPS I observe one health insurance decision (covering one year of behavior) followed by medical care consumption and prices, illness states, and general health status outcomes over the next two years. Therefore, I estimate the structural parameters of the model described in Section 3 using one year of data for each individual. In what follows, I describe the challenges in estimation, discuss identification and unobserved heterogeneity, and construct the estimated likelihood function.

\subsection{Approximating the Future Value of a Medical Care Alternative}

Solution to the optimization problem requires the calculation of an individual's value function for each medical care bundle in each month. According to Equation 3.9, the value of bundle $(v, s, r)$ in month $t, V_{v s r}\left(\Psi_{t}\right)$, is a function of the maximal expected utility in the next month, $V\left(\Psi_{t+1}\right)$, where the future state vector, $\Psi_{t+1}$, is unknown. Thus, in order to calculate $V_{v s r}\left(\Psi_{t}\right)$ in practice, a value $V\left(\Psi_{t+1}\right)$ is needed for every potential outcome of $\Psi_{t+1}$ following every potential history of outcomes $\left(\Psi_{0}, \ldots, \Psi_{t}\right)$. Given the number of months in a year, the number of variables in the state vector, and the fact that several of the state variables are continuous, the number of required future values grows exponentially.

To avoid what Bellman (1957) refers to as the "curse of dimensionality," I use an interpolation technique developed by Keane and Wolpin (1994) to approximate an individual's maximal expected future value in each month. The method works as follows: beginning in the last month of a year, $t=T$, I draw 3500 random outcomes of the state vector, $\Psi_{T}$. I then calculate maximal expected future utility for each draw according to Equation 3.11. By estimating a linear regression of these values on the state variables in $\Psi_{T}$, I generate a mapping from any possible state to expected future values. This mapping can be used in month 
$T-1$ to approximate the maximal expected future value of month $T$, because each alternative in month $T-1$ generates a probability distribution over $\Psi_{T}$. By repeating this process backwards (hence backwards solution), I can solve the model back to month $t=0$.

A related challenge in solving any finite horizon dynamic problem is determining the maximal expected future value in the final period; $Q_{y}\left(\Psi_{0}, H_{1}\right)$ from Equation 3.10. I take a popular approach, which is to formulate a closing function to approximate this value. I assume that this value is determined by a non-stochastic (i.e., no error term) linear function of the state variables entering the first month of the following year, medical care consumed in month $T$, and a vector of parameters. I estimate these parameters as part of the MLE procedure.

\subsection{Identification}

I estimate the following sets of parameters (252 parameters in total)

$$
\begin{array}{ll}
\text { Acute Illness Parameters: } & \Omega^{A}=\left\{\alpha_{00}, \ldots, \alpha_{49}\right\} \\
\text { Chronic Illness Parameters: } & \Omega^{C}=\left\{\delta_{00}, \ldots, \delta_{32}\right\} \\
\text { Health Status Parameters: } & \Omega^{H}=\left\{\psi_{00}, \ldots, \psi_{49}, \kappa\right\} \\
\text { Price Parameters: } & \Omega^{P}=\left\{\lambda_{00}^{x}, \ldots, \lambda_{19}^{x}, \sigma^{x}\right\}_{x \in\{v, s, r\}} \\
\text { Preference Parameters: } & \Omega^{U}=\left\{\omega_{00}, \ldots, \omega_{40}, \beta\right\} \\
\text { DFRE Parameters: } & \Omega^{\mu}=\left\{\left\{\mu_{1}^{k}, \ldots, \mu_{14}^{k}, \theta^{k}\right\}_{k=1}^{4}\right\} \\
\text { Closing Function Parameters: } & \Omega^{F}=\left\{\gamma_{00}, \ldots, \gamma_{11}\right\} \\
\text { Initial Condition Parameters: } & \Omega^{I}=\left\{\tilde{\alpha}_{00}, \ldots, \tilde{\alpha}_{19}, \tilde{\delta}_{00}, \ldots, \tilde{\delta}_{15}, \tilde{\kappa}, \tilde{\psi}_{00}, \ldots, \tilde{\psi}_{16}\right\}
\end{array}
$$

Parameters are identified by individual decisions, the model's economic constraints, and parametric assumptions. Identification of acute illness, chronic illness, and general health status parameters comes from the logit assumption and covariance between the independent and dependent variables. For example, the effect of medical care on illness probabilities is identified by individuals in the same illness state making different medical care consumption decisions and getting different illness outcomes the following month. The identification of parameters in the conditional price distributions is similar, though a gamma distribution is assumed. Initial condition parameters are separately identified from transition parameters by 
four exclusion restrictions and the logit assumption. ${ }^{51}$

The identification of the preference parameters is less straightforward. The marginal (dis)utility of medical care, $\omega_{2}$, is identified through variation in the types of medical care consumed, holding state variables fixed. Insurance cost-sharing features help identify these parameters by often making the expected out-of-pocket cost of medical care equal across the three types. The effect of general health status and illness state on utility, $\omega_{1}$, is identified by the optimization framework and through variation in total monthly medical care consumption for individuals in different wellness states. For example, the marginal disutility of acute illness is identified by differences in total consumption between individuals who are identical except in their acute illness states. An acute illness yields disutility if an individual with an acute illness consumes more medical care (and therefore takes on the costs of consuming care) than an individual without an acute illness, in an attempt to cure the illness. This example also explains why the disutility of chronic illness is not identified and is, therefore, not included as a direct determinant of utility in estimation. Chronic illnesses never subside, meaning the only justification for additional medical care consumption once a chronic illness is obtained is to diminish the marginal negative effect of that chronic illness on the general health status (and acute illness probability). Therefore, disutility from chronic illness is not separately identified from the disutility of poor general health.

Separately identifying moral hazard effects from adverse selection is an important empirical challenge in this research. There is not a single parameter in the model that identifies the effect of moral hazard (instead, simulation techniques are used); however, the curvature parameters in the utility function, $\omega_{0}$, are especially important when calculating moral hazard effects because health insurance cost-sharing characteristics and medical care prices enter the optimization problem by altering the consumption of non-medical goods. The data used in estimation have two features that assist in the identification of these parameters. First,

\footnotetext{
${ }^{51}$ Income last year, an indicator for missing last years income, veteran status, and foreign birth status are included in the estimation of initial condition probabilities but not in transition probabilities.
} 
twelve medical care decisions, often made at different points in the medical care price distribution, are observed for each individual. The covariance between these decisions and the out-of-pocket cost of medical care helps to identify the curvature parameters. However, the out-of-pocket cost of medical care is endogenous because health insurance is chosen by an individual with expectations of future medical care consumption (adverse selection). A second data feature helps to mitigate this problem. Much like the data used by Cardon and Hendel (2001), each individual in the sample selects health insurance from a different set of alternatives. The degree of variation in the alternative sets is widened by the employer's role in determining the out-of-pocket premium paid for a plan. This variation ensures that individuals with similar expectations of future medical care consumption select different health insurance plans and ultimately face different out-of-pocket medical care costs. If insurance alternative sets are determined exogenously, then the variation in alternative sets reduces the endogeneity of out-of-pocket medical care costs.

Modeling the observed health transitions also helps the model separate selection effects from price effects. In general, the key unobservable that contributes to the unexplained correlation between insurance generosity and annual medical care expenditure is an individual's expectation of future health. If an individual expects poor future health outcomes then he purchases generous coverage and (often) has high medical care expenditures. Rather than using parametric assumptions and the optimization framework to capture heterogeneity in the health expectations that lead to adverse selection, I model observed health and illness outcomes over the health insurance year. By modeling these outcomes and solving the problem backwards, an individual's expectations of future health outcomes are modeled explicitly and impact the valuation of each health insurance plan.

\subsection{Unobserved Heterogeneity}

I capture unobserved permanent individual heterogeneity in the model using a discrete factor random effects method (DFRE). The DFRE approach avoids restrictive distributional 
assumptions by allowing the distribution of unobserved heterogeneity to be approximated by a discrete step-wise function (Heckman and Singer 1984; Mroz and Guilkey 1992). Mroz (1999), and more recently Guilkey and Lance (2013), use Monte Carlo simulation in a twoequation setting to show that when the true error distribution is joint normal, DFRE estimates are comparable to those derived using the correct distribution. However, when the true error distribution is not normal, the DFRE outperforms all other (tested) estimation methods.

In this model, illness and general health outcomes, medical care prices, and medical care decisions are each partially determined by unobservables (i.e., an error term), which can be decomposed into two components. The first component, a non-linear discrete factor $\mu^{k}$, represents individual-specific persistent unobserved heterogeneity. The second component is the remaining i.i.d. serially-uncorrelated random error. The population is assumed to have $K$ unobserved types, which are drawn from discrete distribution $\Theta$. The estimation technique determines the value of each discrete factor, $\left\{\mu_{1}^{k}, \ldots, \mu_{14}^{k}\right\}$, for $K-1$ types ( $K-1$ because identification requires that one type has all discrete factors set to zero) and the probability of each type, $\theta^{k}$; where $\sum_{k=1}^{K} \theta^{k}=1$.

In addition to improving the fit of the model, the DFRE method captures unobserved heterogeneity that may induce adverse selection. For example, an individual with an unobserved (to the econometrician) health condition is also more susceptible to acute illness, chronic illness, poor general health status, and greater medical care consumption. The estimation procedure identifies this unobserved heterogeneity type by unexplained correlation between the dependent variables and allows the type to influence his valuation of each health insurance plan.

\subsection{Estimation Procedure}

I estimate the model's parameters, $\Omega$, using a nested fixed point solution algorithm (Rust 1987). The inner algorithm solves the dynamic programming problem for a given set of parameters and for each mass point in the unobserved heterogeneity distribution. The outer 
algorithm uses the resulting probabilities and densities to calculate the likelihood function, $L(\Omega)$, and attempts to improve the likelihood value using a BHHH gradient method (Berndt, Hall, Hall, and Hausman 1974). Standard errors are taken from the main diagonal of the BHHH (i.e., outer product of the gradient) matrix, which approximates the negative of the inverse Hessian.

An individual contributes to the likelihood function the product of his observed illness state, general health status, medical care price, medical care choice, and insurance choice probabilities. General health status and illness state probabilities take on closed forms due to the logit assumptions in Section 3. I assume that prices are gamma distributed so that probability density function values $f^{v}(\cdot), f^{s}(\cdot)$, and $f^{r}(\cdot)$ of the following form enter the likelihood function

$$
f(p ; \sigma, \xi)=\frac{1}{\Gamma(\sigma) \xi \sigma} p^{\sigma-1} e^{\frac{-p}{\xi}}
$$

where $\Gamma(\cdot)$ is the gamma function and $\xi$ and $\sigma$ are the scale and shape parameters of the gamma distribution, respectively. I parameterize $\xi$ such that $\xi=\Phi_{t} * \lambda$, which allows state variables within the model to affect the scale of the price distributions, but not the shape.

I assume $\epsilon_{t}^{v s r}$ and $\phi_{y}^{j}$ each follow a Type 1 Extreme Value distribution. This assumption simplifies estimation in two ways. First, it can be shown that when $\epsilon_{t}^{v s r}$ is Type 1 Extreme Value the expectation in Equation 3.11 is equal to

$$
V\left(\Psi_{t} \mid \mu^{k}, I_{y}^{j}\right)=E C+\ln \left(\sum_{v=0}^{V} \sum_{s=0}^{S} \sum_{r=0}^{1} \exp \left(\bar{V}_{v s r}\left(\Psi_{t} \mid \mu^{k}, I_{y}^{j}\right)\right)\right) \quad \forall t
$$

where $E C$ is Euler's Constant. The assumption simplifies solution to the optimization problem, as calculation/simulation of a $(V * S * 2)-1$ dimensional integral would be required if another popular continuous distribution without a closed form (e.g., normal) were chosen (see Keane and Wolpin 1994). Second, the additive Type 1 Extreme Value distribution 
assumptions yield choice probabilities that have the following closed form structures.

$$
\begin{gathered}
P\left(d_{t}^{v s r}=1 \mid \Psi_{t}, \mu^{k}, I_{y}^{j}\right)=\frac{\exp \left(\bar{V}_{v s r}\left(\Psi_{t} \mid \mu^{k}, I_{y}^{j}\right)\right)}{\sum_{v^{\prime}=0}^{V} \sum_{s^{\prime}=0}^{S} \sum_{r^{\prime}=0}^{1} \exp \left(\bar{V}_{v^{\prime} s^{\prime} r^{\prime}}\left(\Psi_{t} \mid \mu^{k}, I_{y}^{j}\right)\right)} \quad \forall t, \forall v s r \\
P\left(I_{y}^{j}=1 \mid \Psi_{0}, \mu^{k}\right)=\frac{\exp \left(\bar{Q}_{j}\left(\Psi_{0}, \mu^{k}\right)\right)}{\sum_{j^{\prime}=0}^{J^{i}} \exp \left(\bar{Q}_{j^{\prime}}\left(\Psi_{0}, \mu^{k}\right)\right)} \quad \forall y, \forall j
\end{gathered}
$$

The likelihood contribution for individual $i$ in month $t$ conditional on $\mu^{k}$ and observed $H_{t}$ is

$$
\begin{aligned}
L_{i t}\left(\Omega \mid \mu^{k}, I_{y}^{j}, H_{t}=h\right) & =\left(\left[\pi_{t}^{0}\left(\cdot \mid \mu^{k}\right)\right]^{1-A_{t}}\left[\pi_{t}^{1}\left(\cdot \mid \mu^{k}\right)\right]^{A_{t}}\right)\left(\left[\gamma_{t}^{0}\left(\cdot \mid \mu^{k}\right)\right]^{1-C_{t}}\left[\gamma_{t}^{1}\left(\cdot \mid \mu^{k}\right)\right]^{C_{t}}\right)^{1-C_{t-1}} \\
& \prod_{v=0}^{V} \prod_{s=0}^{S} \prod_{r=0}^{1}\left[f^{v}\left(p_{t}^{v} \mid \mu^{k}\right)^{\left[1-d_{t}^{0 s r}\right]} f^{s}\left(p_{t}^{s} \mid \mu^{k}\right)^{\left[1-d_{t}^{v 0 r}\right]} f^{r}\left(p_{t}^{r} \mid \mu^{k}\right)^{\left[1-d_{t}^{v s 0}\right]}\right. \\
& \left.P\left(d_{i t}^{v s r}=1 \mid \Psi_{t}, I_{y}^{j}, \mu^{k}\right) \prod_{h^{\prime}=0}^{2} \eta_{t+1}^{h^{\prime}}\left(\Psi_{t}, d_{t}^{v s r} \mid \mu^{k}\right)^{\mathbb{1}_{H_{t+1}}=h^{\prime}}\right]^{d_{t}^{v s r}} .
\end{aligned}
$$

The first row contains the illness state contribution for month $t$. The price densities are in the second row. The $\left[1-d_{t}^{0 s r}\right]$ exponent ensures that price of a doctor visit in month $t$ contributes to the likelihood function only if an individual actually visits the doctor, which is the only time that I observe this price (this is true for each type of care). The third row contains both month $t$ choice probabilities and the probabilities of transitioning to a new general health status entering month $t+1$. To control for endogenous initial conditions, $L_{i 1}\left(\Omega \mid \mu^{k}, I_{y}^{j}, H_{t}=h\right)$ appears as above with the first row replaced by

$$
\prod_{h=0}^{2}\left(\tilde{\eta}_{1}^{h}\left(\cdot \mid \mu^{k}\right)^{\mathbb{1}_{H_{1}=h}}\right)\left(\left[\tilde{\pi}_{1}^{0}\left(\cdot, h \mid \mu^{k}\right)\right]^{1-A_{1}}\left[\tilde{\pi}_{1}^{1}\left(\cdot, h \mid \mu^{k}\right)\right]^{A_{1}}\right)\left(\left[\tilde{\gamma}_{1}^{0}\left(\cdot, h \mid \mu^{k}\right)\right]^{1-C_{1}}\left[\tilde{\gamma}_{1}^{1}\left(\cdot, h \mid \mu^{k}\right)\right]^{C_{1}}\right) .
$$

These initial probabilities $(\tilde{\eta}, \tilde{\pi}, \tilde{\gamma})$ are separately estimated from transition probabilities (with exclusion restrictions) and are allowed to vary by modeled unobserved heterogeneity.

I write $L_{i t}\left(\Omega \mid \mu^{k}, I_{y}^{j}, H_{t}=h\right)$ conditional on $\left(H_{t}=h\right)$ because I only observe general 
health status in months where an interview is conducted. ${ }^{52}$ Therefore, I integrate over the general health status distribution in months where health is missing. The total likelihood contribution for individual $i$ conditional on $\mu^{k}$ is then written

$$
L_{i}\left(\Omega \mid \mu^{k}\right)=\prod_{j=1}^{J^{i}}\left[P\left(I_{i y}^{j}=1 \mid \Psi_{0}, \mu^{k}\right) \prod_{t=1}^{T} L_{i t}\left(\Omega \mid \mu^{k}, I_{y}^{j}\right)\right]^{I_{i y}^{j}} .
$$

The contribution of individual $i$ unconditional on the unobserved heterogeneity is

$$
L_{i}(\Omega)=\sum_{k=1}^{K} \theta^{k} L_{i}\left(\Omega \mid \mu^{k}\right)
$$

\footnotetext{
${ }^{52}$ For most individual's, I observe 4 measures of self-reported health. The first report, which is used for the initial condition, is taken from the NHIS report provided in the previous year. The second and third reports are taken from MEPS interviews conducted during the health insurance year. The fourth report is take from the third MEPS interview, which occurs during the insurance year for some, but occurs after the end of the insurance year for others. I use the third interview self-reported health level for the the last month general health outcome even if the interview occurs past the end of the health insurance year.
} 


\section{RESULTS}

This section begins with parameter estimates. I then discuss model fit, the estimated effect of moral hazard, and model predictions under several counterfactual situations.

\subsection{Parameter Estimates}

Table 6.1 reports estimated preference parameters. A constant relative risk aversion (CRRA) parameter, $R A$, can be calculated for each individual using $\left(\omega_{00}, \omega_{01}, \omega_{02}, \omega_{03}\right) .{ }^{53}$ Non-whites and males are found to be significantly less risk averse than whites and females. Risk aversion is increasing in age. ${ }^{54} \mathrm{~A} 40$ year-old white male has $R A=0.866$, whereas a 40 year-old white female has $R A=0.879$. At the sample mean, $R A=0.925$. These estimates are between the Blau and Gilleskie (2008) estimate of 0.96 and the Imai and Keane (2004) and Sauer (2012) estimate of 0.74; none of which allow for heterogeneity in risk preferences by demographic group. Parameters $\left(\omega_{10}, \ldots, \omega_{12}, \omega_{30}, \ldots, \omega_{32}\right)$ capture disutility from poor health and acute illness. Note that an individual in good (but not excellent) health receives a contribution of $\omega_{10}$ to his utility function, while an individual in poor health receives a contribution of $\omega_{10}+\omega_{11}$. The direct utility effect of medical care consumption is captured by $\left(\omega_{20}, \ldots, \omega_{24}, \omega_{33}, \ldots, \omega_{38}\right) .{ }^{55}$ Interpreting the linear and quadratic consumption terms is

\footnotetext{
${ }^{53}$ The CRRA risk aversion parameter is calculated as $R A=\left[1-\omega_{00}-\omega_{01} *\right.$ age $-\omega_{02} *$ nonwhite $-\omega_{03} *$ male]. Age is scaled in estimation so that the youngest individual included (19) has an age of 0 . A 40 year-old, then, has an age of 21 .

${ }^{54}$ There is a large literature on the relationship between risk aversion and demographics. Croson and Gneezy (2009) summarize the literature on gender and risk, which consistently finds women to be more risk averse. Eckel (2008) also find that women are more risk averse than men, but find no significant race effect and mixed age effects. Rosen, Tsai, and Downs (2003) find gender and race effects that are similar to those that I find, but do not study age.

${ }^{55}$ These parameters can be interpreted as the net direct effect of medical care consumption on utility. The physical, psychological, and time cost of medical care consumption may have negative effects on these parameters. However, some individuals may enjoy consuming medical care, independent of its productive health
} 
not useful without also considering the discrete factor terms discussed in the next paragraph. However, these parameters do reveal that men have significantly lower preferences for doctor visits and prescription drugs than women. Further, preferences for doctor visits are decreasing in age while preferences for prescription drugs are increasing in age. The final parameter of the utility function reflects the disutility of each dollar of negative consumption (i.e., outspending monthly income, requiring an individual to use savings or to borrow) and is not currently estimated.

The parameters in Table 6.2 describe the discrete step-wise function used to approximate the joint distribution of unobservables in the model. The technique uses the estimation procedure to group the population of individuals by unobservables and estimate the relative effect these groups have on the model's probabilities, along with the probability of being in a particular group. ${ }^{56}$ For identification, one group's mass points and probability parameter must be normalized to zero (though not necessarily the same group). I fix both for group 1. Estimation reveals that $7.5 \%$ of the population is in group $1[1-0.576-0.170-0.179=0.075]$. The preference parameters in Table 6.1 fully describe preferences for this group. One oddity of this group is that its females derive utility directly from prescription drug consumption $\left(\omega_{24}\right)$. The other three groups are similar in health and in medical care preferences $\left(\mu_{1}, \ldots, \mu_{6}\right.$ in Table 6.2). In comparison to group 1, these individuals are less likely to get an acute illness, more likely to get a chronic illness, and are in a better general health status. They also receive greater disutility from all 3 medical care types. Of the four groups, group 2 seems to represent individuals who are in the best state of (unobserved) wellness, with the lowest preferences for medical care. Group 1 represents individuals with the worst state of (unobserved) wellness, effects, which has a positive effect on these parameters.

\footnotetext{
${ }^{56}$ The number of points of support (or groups) is chosen by the econometrician. It is suggested by Mroz (1999) that this number should be chosen using an "upwards-testing approach based on the increase in the quasilikelihood function value when one adds an additional point of support." This technique is cost prohibitive in this work because additional mass points increase estimation time substantially. Instead, I use an upwards-testing approach that requires a significant improvement in the likelihood function and an improvement in model fit to add additional points of support. I arrive at 4 mass points in the current model.
} 
Table 6.1: Preference Parameter Estimates

\begin{tabular}{|c|c|c|c|}
\hline & Parameter & Estimate & S.E. \\
\hline \multicolumn{4}{|l|}{ Utility Function } \\
\hline RA constant & $\omega_{00}$ & 0.1785 & 0.0073 \\
\hline RA age & $\omega_{01}$ & -0.0030 & 0.0003 \\
\hline RA non-white (black or Hispanic) & $\omega_{02}$ & 0.0324 & 0.0055 \\
\hline RA male & $\omega_{03}$ & 0.0126 & 0.0059 \\
\hline less than excellent health & $\omega_{10}$ & -18.5305 & 2.9335 \\
\hline less than good health & $\omega_{11}$ & -13.8780 & 1.8515 \\
\hline acute illness & $\omega_{12}$ & -17.2095 & 3.3050 \\
\hline doctor visits & $\omega_{20}$ & -0.2258 & 0.0814 \\
\hline doctor visits $^{2}$ & $\omega_{21}$ & 0.0355 & 0.0053 \\
\hline hospital days & $\omega_{22}$ & -2.8720 & 0.2164 \\
\hline hospital days ${ }^{2}$ & $\omega_{23}$ & 0.4089 & 0.0422 \\
\hline any $\mathrm{Rx}$ consumption & $\omega_{24}$ & 1.8402 & 0.3495 \\
\hline less than excellent health*age & $\omega_{30}$ & 0.0280 & 0.0223 \\
\hline less than good health*age & $\omega_{31}$ & -0.0031 & 0.0264 \\
\hline acute illness*age & $\omega_{32}$ & -0.0139 & 0.0170 \\
\hline doctor visits*age & $\omega_{33}$ & -0.0080 & 0.0009 \\
\hline doctor visits*male & $\omega_{34}$ & -0.1888 & 0.0215 \\
\hline hospital days*age & $\omega_{35}$ & 0.0014 & 0.0030 \\
\hline hospital days*male & $\omega_{36}$ & 0.0811 & 0.0586 \\
\hline any $\mathrm{Rx}$ consumption*age & $\omega_{37}$ & 0.0411 & 0.0047 \\
\hline any $\mathrm{Rx}$ consumption*male & $\omega_{38}$ & -1.5221 & 0.0869 \\
\hline negative consumption ${ }^{\dagger}$ & $\omega_{40}$ & 0.0010 & $*$ \\
\hline \multicolumn{4}{|l|}{ Other } \\
\hline discount factor ${ }^{\dagger}$ & $\beta$ & 0.996 & $*$ \\
\hline log-likelihood value $\ddagger$ & $L(\Omega)$ & -78920.570 & $*$ \\
\hline
\end{tabular}

with the highest preferences for medical care. Groups 2 and 3 lie somewhere in the middle.

It is well known in the health economics literature that estimating the productive effects of medical care (on health and/or illness) is challenging. The unconditional correlation between medical consumption and wellness is usually negative because an individual consumes more medical care when sick. The negative correlation likely reflects bias associated with selection into consumption and omitted health or medical care heterogeneity. In this research, I address the first issue by modeling medical care consumption (i.e., the heath input) and allowing for common unobserved individual heterogeneity that affects both medical care decisions and 
Table 6.2: Permanent Unobserved Heterogeneity Parameter Estimates

\begin{tabular}{|c|c|c|c|c|c|c|c|}
\hline & \multirow[t]{2}{*}{ Param. } & \multicolumn{2}{|c|}{ Type 2} & \multicolumn{2}{|c|}{ Type 3} & \multicolumn{2}{|c|}{ Type 4} \\
\hline & & est. & s.e. & est. & s.e. & est. & s.e. \\
\hline \multicolumn{8}{|l|}{ Mass Point Location } \\
\hline acute illness probability & $\mu_{1}$ & -0.955 & 0.087 & -0.282 & 0.085 & -0.410 & 0.090 \\
\hline chronic illness probability & $\mu_{2}$ & 4.782 & 1.059 & 4.959 & 1.055 & 4.625 & 1.052 \\
\hline health status probability & $\mu_{3}$ & 0.141 & 0.055 & 0.168 & 0.055 & 0.050 & 0.053 \\
\hline doctor visit preference (linear) & $\mu_{4}^{a}$ & -0.859 & 0.065 & -0.400 & 0.057 & -0.333 & 0.055 \\
\hline doctor visit preference (squared) & $\mu_{4}^{b}$ & 0.064 & 0.009 & 0.016 & 0.009 & -0.005 & 0.008 \\
\hline hospital day preference (linear) & $\mu_{5}^{a}$ & -1.076 & 0.191 & -0.041 & 0.087 & -1.371 & 0.217 \\
\hline hospital day preference (squared) & $\mu_{5}^{b}$ & 0.296 & 0.056 & 0.093 & 0.037 & 0.313 & 0.065 \\
\hline any $\mathrm{Rx}$ preference & $\mu_{6}$ & -6.026 & 0.269 & -1.569 & 0.257 & -1.117 & 0.262 \\
\hline doctor visit price distribution & $\mu_{7}$ & 0.034 & 0.028 & -0.025 & 0.032 & -0.187 & 0.031 \\
\hline hospital day price distribution & $\mu_{8}$ & 0.021 & 0.047 & -0.112 & 0.122 & 0.078 & 0.150 \\
\hline monthly Rx price distribution & $\mu_{9}$ & -1.658 & 0.026 & -2.104 & 0.020 & -1.019 & 0.021 \\
\hline initial acute illness probability & $\mu_{10}$ & -1.077 & 0.336 & 0.117 & 0.366 & 0.091 & 0.372 \\
\hline initial chronic illness probability & $\mu_{11}$ & -1.624 & 0.448 & 0.175 & 0.466 & 0.397 & 0.495 \\
\hline initial health status probability & $\mu_{12}$ & 0.905 & 0.298 & 0.761 & 0.322 & 0.661 & 0.329 \\
\hline \multicolumn{8}{|l|}{ Type Probabilities ${ }^{\dagger}$} \\
\hline parameter estimate & $\theta$ & 2.038 & 0.143 & 0.803 & 0.159 & 0.863 & 0.159 \\
\hline type probability & & \multicolumn{2}{|c|}{57.6} & \multicolumn{2}{|c|}{17.0} & \multicolumn{2}{|c|}{17.9} \\
\hline
\end{tabular}

health outcomes. I address the second issue by simultaneously controlling for 3 measures of health (i.e., acute illness, chronic illness, and general health status) and by allowing the productivity of each type of medical care to vary by illness state. Table 6.3 reports acute and chronic illness probability parameter estimates and Table 6.4 contains general health status probability parameter estimates. For each health outcome, parameter estimates tell a consistent story of wellness persistence (i.e., if an individual has ailment $\mathrm{A}$ in month $t$ then he is more likely to have ailment $\mathrm{A}$ in month $t+1$ ) and cross-wellness effects (i.e., if an individual has ailment $\mathrm{A}$ in month $t$ then he is more likely to have ailment $\mathrm{B}$ in month $t+1$ ). The effectiveness of medical care varies by care type and health measure. All medical care types are productive in preventing and curing an acute illness. Only prescription drugs $\left(\delta_{32}\right)$ are productive in preventing chronic illness. In fact, doctor visits and hospital days are found to 
Table 6.3: Illness Probability Parameter Estimates

\begin{tabular}{lcrc}
\hline & Parameter & Estimate & SE \\
\hline Acute Illness ${ }^{\dagger}$ & & & \\
constant & $\alpha_{00}$ & -1.6066 & 0.1690 \\
male & $\alpha_{10}$ & -0.4522 & 0.0399 \\
non-white (black or Hispanic) & $\alpha_{11}$ & -0.1844 & 0.0413 \\
education (highest grade completed) & $\alpha_{12}$ & 0.0154 & 0.0073 \\
age & $\alpha_{13}$ & -0.0046 & 0.0041 \\
lives in a MSA & $\alpha_{14}$ & -0.0015 & 0.0169 \\
income (in 1996 dollars) & $\alpha_{15}$ & 0.0028 & 0.0011 \\
acute illness & $\alpha_{20}$ & 2.7578 & 0.0824 \\
chronic illness & $\alpha_{21}$ & 0.2383 & 0.0802 \\
less than excellent health & $\alpha_{22}$ & -0.0050 & 0.0472 \\
less than good health & $\alpha_{23}$ & 0.4982 & 0.1235 \\
doctor visits & $\alpha_{30}$ & -0.1120 & 0.0210 \\
hospital days & $\alpha_{31}$ & -0.1346 & 0.0284 \\
Rx consumption & $\alpha_{32}$ & -0.2751 & 0.0476 \\
acute illness*doctor visits & $\alpha_{40}$ & 0.0444 & 0.0151 \\
acute illness*hospital days & $\alpha_{41}$ & 0.0557 & 0.0240 \\
acute illness*Rx consumption & $\alpha_{42}$ & 0.1069 & 0.0448 \\
chronic illness*doctor visits & $\alpha_{43}$ & 0.0637 & 0.0141 \\
chronic illness*hospital days & $\alpha_{44}$ & 0.0867 & 0.0241 \\
chronic illness*Rx consumption & $\alpha_{45}$ & 0.0108 & 0.0099 \\
acute illness*age & $\alpha_{46}$ & 0.0238 & 0.0034 \\
chronic illness*age & $\alpha_{47}$ & 0.0034 & 0.0031 \\
less than excellent health*age & $\alpha_{48}$ & 0.0021 & 0.0044 \\
less than good health*age & $\alpha_{49}$ & -0.0082 & 0.0049 \\
\hline Chronic Illness & & & \\
constant & $\delta_{00}$ & -9.2185 & 1.0961 \\
male & $\delta_{31}$ & -1.9013 & 0.1305 \\
non-white (black or Hispanic) & $\delta_{32}$ & -0.0243 & 0.0203 \\
education (highest grade completed) & $\delta_{11}$ & -0.0746 & 0.0250 \\
age & $\delta_{12}$ & 0.0097 & 0.0052 \\
lives in a MSA & $\delta_{13}$ & -0.0130 & 0.0019 \\
income (in 1996 dollars) & $\delta_{14}$ & 0.2156 & 0.0431 \\
acute illness & $\delta_{15}$ & 0.0011 & 0.0006 \\
less than excellent health & $\delta_{20}$ & 0.1573 & 0.0331 \\
less than good health & 0.6176 & 0.1283 \\
doctor visits & 0.5753 & 0.1241 \\
hospital days & 0.2018 & 0.0140 \\
Rx consumption & & & \\
\hline
\end{tabular}

$\dagger$ Month indicators are included in regression but are not reported here. 
Table 6.4: General Health Status Probability Parameter Estimates

\begin{tabular}{lcrc}
\hline & Parameter & Estimate & SE \\
\hline General Health Status & & & \\
constant & $\psi_{00}$ & 4.3994 & 0.1663 \\
male & $\psi_{10}$ & -0.0864 & 0.0333 \\
non-white (black or Hispanic) & $\psi_{11}$ & 0.0097 & 0.0329 \\
education (highest grade completed) & $\psi_{12}$ & 0.0165 & 0.0061 \\
age & $\psi_{13}$ & -0.0066 & 0.0039 \\
lives in a MSA & $\psi_{14}$ & -0.0004 & 0.0042 \\
income (in 1996 dollars) & $\psi_{15}$ & 0.0049 & 0.0011 \\
acute illness & $\psi_{20}$ & -0.1424 & 0.0573 \\
chronic illness & $\psi_{21}$ & -0.4984 & 0.0656 \\
less than excellent health & $\psi_{22}$ & -2.8126 & 0.1580 \\
less than good health & $\psi_{23}$ & -2.9158 & 0.1527 \\
doctor visits & $\psi_{30}$ & -0.1231 & 0.0137 \\
hospital days & $\psi_{31}$ & -0.1989 & 0.0228 \\
Rx consumption & $\psi_{32}$ & -0.4861 & 0.0526 \\
acute illness*doctor visits & $\psi_{40}$ & 0.0711 & 0.0088 \\
acute illness*hospital days & $\psi_{41}$ & 0.0896 & 0.0147 \\
acute illness*Rx consumption & $\psi_{42}$ & 0.1282 & 0.0238 \\
chronic illness*doctor visits & $\psi_{43}$ & 0.0330 & 0.0064 \\
chronic illness*hospital days & $\psi_{44}$ & 0.0582 & 0.0151 \\
chronic illness*Rx consumption & $\psi_{45}$ & 0.4655 & 0.0492 \\
acute illness*age & $\psi_{46}$ & -0.0005 & 0.0024 \\
chronic illness*age & $\psi_{47}$ & -0.0005 & 0.0024 \\
less than excellent health*age & $\psi_{48}$ & 0.0103 & 0.0055 \\
less than good health*age & $\psi_{49}$ & -0.0159 & 0.0051 \\
cut-point & $\kappa$ & 3.4409 & 0.0690 \\
\hline
\end{tabular}

Month indicators are included in estimation but are not reported here.

increase the likelihood of chronic illness, though the effect is small. ${ }^{57}$ Medical care does little to improve an individual's general health status. Neither doctor visits $\left(\psi_{30}, \psi_{40}, \psi_{43}\right)$ nor hospital days $\left(\psi_{31}, \psi_{41}, \psi_{44}\right)$ positively impact general health status transitions. However, acute and chronic illness does impact transitions between the healths status outcomes. Prescription drugs maintain or improve general health status when an individual has a chronic and acute

\footnotetext{
${ }^{57}$ Medical care consumption types are not interacted with illness states in the chronic illness probability because there is not enough variation in the data to identify the parameters. Keep in mind that $39 \%$ of the population enters the insurance year with a chronic illness, so they provide no contribution to the monthly probability of chronic illness contraction. By years end, $50 \%$ of the total population has a chronic illness, meaning that of the (roughly) 8,100 chronic illness probability contributions to the likelihood function, only 135 reflect chronic illness contraction.
} 
illness $\left(\psi_{32}, \psi_{42}, \psi_{45}\right) .^{58}$

Price, initial condition, and closing function parameters can be found in Tables A.2, A.3, and A.4, which are located in Appendix A.

\subsection{Model Fit}

I use simulation techniques to assess the fit of the model. Using observed initial conditions and insurance offer sets, I simulate annual insurance and monthly medical care decisions, monthly illness state and general health status transitions, and monthly medical care prices for each of the 1232 observed individuals. Using random draws from the appropriate error distributions, I replicate the simulation 100 times. I compare the average across simulated replications with their observed counterparts. Standard errors have not yet been calculated for model fit comparisons.

A good indicator of model fit is total annual expenditure, as it requires proper fit of all three consumption and price distributions. Figure 6.1 shows both observed and simulated annual expenditure distributions. The figure reveals an underprediction of zero medical care expenditure ( $11 \%$ of the simulated sample vs. $22 \%$ of the observed data). What is not immediately evident from the figure, but is shown in Table 6.5, is that the observed distribution also has a higher mean and standard deviation than the simulated distribution. Matching the observed annual expenditure distribution is a difficult endeavor even in annual expenditure models. The research cited earlier often struggles to explain the same unique features of the observed expenditure distribution seen here; namely, the large mass at zero and the long right tail. In this research, annual medical care expenditure is an outcome that is determined by unit consumption decisions and prices. Table 6.5 shows that the simulated means and standard deviations of all three price distributions are lower than those of the observed distributions. Most importantly, the standard deviation of the simulated doctor visit price distribution is

\footnotetext{
${ }^{58}$ While seemingly counter-intuitive, it is not too surprising that medical care consumption has few positive effects on general health status given that the measure is self-reported. Medical care consumption could serve as a signal/reminder to an individual that he is not in perfect health, making a negative report more likely.
} 
much lower than the standard deviation of the observed distribution. Experimentation has shown that when the price parameters are adjusted so that the simulated means and standard deviations more closely match the observed, the mean and standard deviation of the simulated expenditure distribution improves substantially. This improvement comes at little or no cost to the fit of other simulated variables. Thus, the fit of the price and expenditure distributions may benefit from an alternative distributional assumptions for prices. ${ }^{59}$

Figure 6.1: Annual Medical Care Expenditure

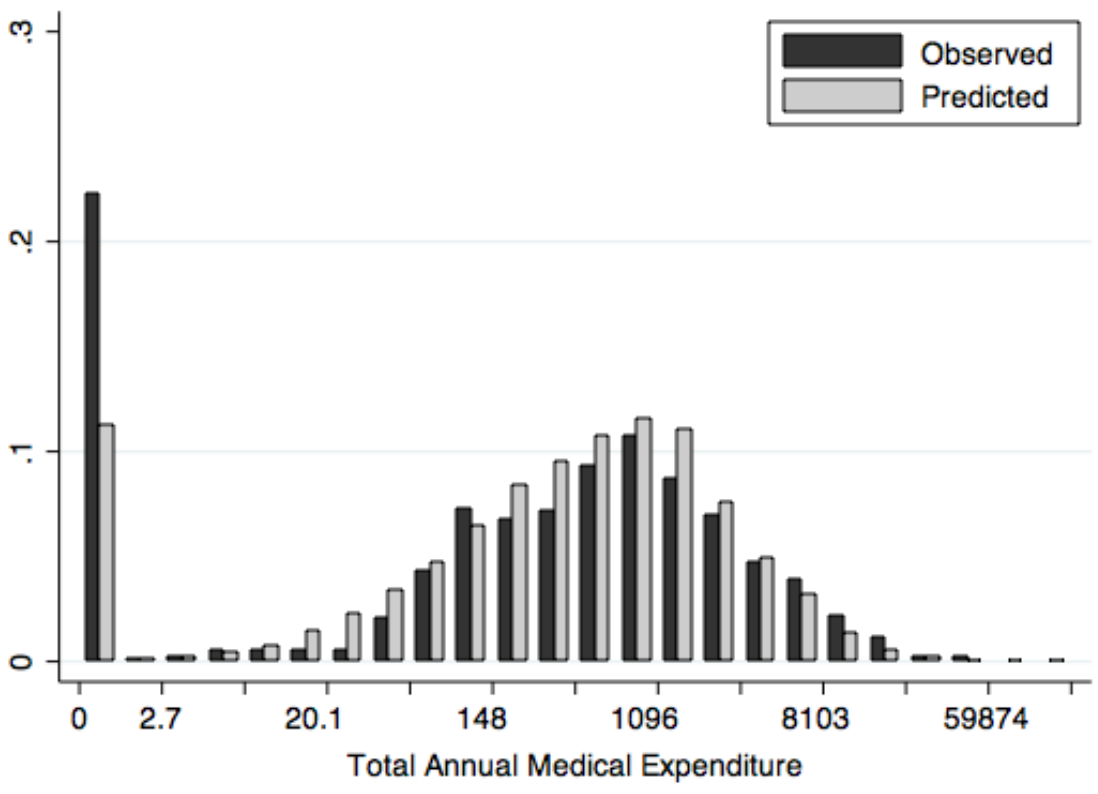

This figure compares observed and simulated annual expenditure distributions. The horizontal axis is log-scaled, so that markers are spaced $\exp (1.0)$ apart.

Simulated annual medical care consumption and monthly consumption by illness state appear to match the observed data well in Table 6.5. However, the full consumption distributions displayed in Table 6.6 mirror a problem with the expenditure distribution; the model is

\footnotetext{
${ }^{59}$ The model presented in this research assumes that all three prices are drawn from gamma distributions. An earlier version of the model was estimated assuming log-normal distributions, which provided a worse fit for the hospital price distribution and prescription drug price distribution but a better fit for the doctor visit price distribution. The model is currently being reestimated assuming the doctor visit price distribution is log-normal and the hospital day and prescription drug price distributions are gamma. My preference would be to use a conditional density estimator like Gilleskie and Mroz (2004), but the estimator requires a significant increase in the number of estimated parameters, which could increase estimation time prohibitively.
} 
Table 6.5: Observed and Simulated Outcomes

\begin{tabular}{|c|c|c|c|c|c|c|}
\hline & \multicolumn{3}{|c|}{ Observed } & \multicolumn{3}{|c|}{ Simulated } \\
\hline & mean & median & s.d. & mean & median & s.d. \\
\hline Total Annual Expenditure & 1470.09 & 347.58 & 3654.74 & 1243.80 & 425.25 & 2761.98 \\
\hline \multicolumn{7}{|l|}{ Medical Care Prices ${ }^{\dagger}$} \\
\hline doctor visit price & 90.26 & 55.00 & 159.30 & 89.46 & 63.54 & 87.42 \\
\hline hospital day price & 828.10 & 329.60 & 1436.26 & 827.42 & 342.88 & 1364.75 \\
\hline prescription drug price & 74.06 & 41.62 & 112.99 & 67.31 & 37.08 & 90.71 \\
\hline \multicolumn{7}{|l|}{ Medical Care Consumption } \\
\hline annual doctor visits & 4.89 & 2.00 & 8.69 & 4.83 & 2.00 & 6.26 \\
\hline annual hospital days & 0.65 & 0.00 & 2.00 & 0.60 & 0.00 & 1.40 \\
\hline months with Rx cons. & 4.46 & 2.00 & 4.95 & 4.17 & 2.00 & 4.43 \\
\hline$\%$ consuming (any month) & 43.59 & * & * & 44.17 & * & * \\
\hline$\%$ consuming, $A_{i t}=1$ & 71.70 & $*$ & $*$ & 70.79 & $*$ & $*$ \\
\hline$\%$ consuming, $C_{i t}=1$ & 72.89 & $*$ & $*$ & 69.17 & $*$ & $*$ \\
\hline$\%$ consuming, $A_{i t}=C_{i t}=0$ & 9.25 & $*$ & $*$ & 15.07 & $*$ & $*$ \\
\hline \multicolumn{7}{|l|}{ Monthly Health } \\
\hline$\%$ with acute illness & 36.41 & $*$ & $*$ & 34.79 & $*$ & $*$ \\
\hline$\%$ with chronic illness & 44.03 & $*$ & $*$ & 43.75 & $*$ & $*$ \\
\hline$\%$ in excellent health & 32.55 & $*$ & $*$ & 31.03 & $*$ & $*$ \\
\hline$\%$ in good health & 35.82 & $*$ & $*$ & 38.89 & $*$ & $*$ \\
\hline$\%$ in poor health & 31.63 & $*$ & $*$ & 30.08 & $*$ & \\
\hline \multicolumn{7}{|l|}{ Held Insurance Types } \\
\hline$\%$ No Insurance & 9.16 & $*$ & $*$ & 16.52 & $*$ & $*$ \\
\hline$\% \mathrm{HMO}$ & 37.96 & $*$ & $*$ & 33.55 & $*$ & $*$ \\
\hline$\%$ PPO & 10.14 & $*$ & $*$ & 9.89 & $*$ & $*$ \\
\hline$\% \mathrm{FFS}$ & 42.74 & $*$ & $*$ & 40.03 & $*$ & $*$ \\
\hline
\end{tabular}

$\dagger$ Prices are only observed when an individual consumes medical care; thus, the simulated mean and standard deviation are calculated only from individuals consuming care in simulation.

currently unable to generate enough zero-consumers. The largest difference is found for doctor visits. Again, manual manipulation of model parameters shows that if these consumption distributions can shift some mid-range consumers to zero consumption, then the expenditure distribution improves. ${ }^{60}$

Table 6.5 also reveals that simulated monthly health status and illness state probabilities

\footnotetext{
${ }^{60}$ The DFRE method being used does improve the fit of all 3 consumption distributions in the appropriate directions (namely, the model that allows for four unobserved types generates significantly more zero-consumers than the model with only one unobserved type). However, adding additional mass points does not further improve the fit of these distributions. The model is currently being reestimated with a more flexible medical care preference structure to allow for more zero-consumers.
} 
Table 6.6: Observed and Simulated Annual Consumption

\begin{tabular}{lrrrrrrrr}
\hline & \multicolumn{2}{c}{ Doctor visits } & & \multicolumn{2}{c}{ Hospital days } & & \multicolumn{2}{c}{ Prescriptions } \\
\cline { 2 - 3 } \cline { 7 - 8 } & obs. & sim. & & obs. & sim. & & obs. & sim. \\
\hline 0 & 28.22 & 18.94 & & 75.83 & 72.25 & & 35.44 & 30.43 \\
1 & 16.06 & 17.76 & & 12.08 & 16.40 & & 13.06 & 14.36 \\
2 & 12.73 & 12.68 & & 4.87 & 4.46 & & 5.92 & 8.11 \\
$3-5$ & 18.41 & 21.22 & & 4.79 & 5.07 & & 8.14 & 13.09 \\
$6-9$ & 10.46 & 13.88 & & 1.46 & 1.52 & & 7.79 & 12.31 \\
$10+$ & 14.12 & 15.52 & & 0.97 & 0.30 & & 27.66 & 21.69 \\
\hline
\end{tabular}

The table reports annual consumption levels for the observed and simulated samples. The values are the proportion of the samples consuming at each level. For example, the top left entry states that $28.22 \%$ of the observed sample visited a doctor's office zero times during the insurance year. The prescription drug levels are measured in consumption months.

match the observed data well. With regard to the fit of health insurance type, the current parameter estimates predict that too many individuals choose to be uninsured. The overprediction is a result of the expenditure distributions' thin right tail. When the variances of medical care prices are manually increased to match the data, not only does the mean and variance of the expenditure distribution increase but the likelihood of being insured does as well.

\subsection{Moral Hazard}

Empirical studies of moral hazard often quantify the effect of insurance possession on medical care consumption and, at times, measure the welfare implications of this additional consumption. In this dissertation, I do the former and discuss the latter in Appendix D. A well known measure of the relationship between a health insurance and medical care demand is the single price/co-insurance elasticity of medical care demand, which was made popular by RAND Health Insurance Experiment (HIE) researchers. In more recent work, researchers have calculated the proportion of total annual expenditure (when insured) that is caused by insurance possession (Bajari et al. 2013) as a measure of moral hazard effects. My preferred measure, and the measure that I focus on in this research, is the average increase in total annual expenditure due to insurance coverage (Einav et al. 2013). 
To be more precise, I calculate the percentage increase in mean total annual medical care expenditure in the population when individuals are moved from an uninsured state into their preferred health insurance plan. I calculate this statistic in two stages. In the first stage, I use the model to forward simulate behavior assuming that all individuals are without insurance. In the second stage, I simulate the model while forcing all individuals to select a health insurance plan from the alternative set offered by their employer. Mean predicted expenditure without coverage is $\$ 668$. Row 1 of Table 6.7 reports the predicted increase in total annual medical care expenditure when individuals move from no insurance to their preferred plan. Mean expenditure increases by $\$ 615$, or by $92 \%$; however, these moral hazard effects vary widely across the population and are driven by those with very large increases in expenditure. If the top $1 \%$ of additional spenders (i.e., those with spending increases in excess of $\$ 10,515$ ) are removed, then the mean expenditure increases $65 \%$ in response to insurance acquisition. ${ }^{61}$ Furthermore, $44 \%$ of the sample does not increase its spending at all in response to coverage. In Row 2, I report the estimated effect of moral hazard on expenditure when individuals are moved from no coverage to full coverage, which allows for comparisons across papers. The out-of-pocket insurance premium is assumed to be zero with full coverage.

Table 6.7: Predicted Effect of Insurance Possession on Medical Care Expenditure

\begin{tabular}{lcccccc}
\hline & Mean & 25th & 50th & 75th & 90th & 95th \\
\hline $\begin{array}{l}\text { Expenditure increase going from } \\
\text { no coverage to preferred coverage }\end{array}$ & 615 & 0 & 22 & 300 & 1,445 & 3,122 \\
no coverage to full coverage & 741 & 0 & 84 & 583 & 2,027 & 3,806 \\
\hline
\end{tabular}

As stated above, there are many different measures moral hazard effects reported in the literature. By simulating behavior under various insurance conditions, I am able to calculate

\footnotetext{
${ }^{61}$ This begs the question, why are these few individuals consuming so much more care in response to coverage? In the model, large expenditures are driven by high medical care price draws. Thus, the strong reaction to coverage by some is often driven by a modest increase in consumption that results in a very large expenditure increase due to the price shock. Imagine, for example, a sprained ankle. Without insurance an individual may wait several days to see if the injury will heal on its own. With insurance, the individual may go to the emergency room immediately, which leads to a room charge, an x-ray, and the purchase of a removable cast, each of which can carry a substantial cost.
} 
several of these measures using my model and data. These comparisons are reported in Table 6.8. As presented in Row 1, Manning et al. (1987) use the experimental RAND HIE data and "two- and four- part" models to estimate a co-insurance (arc) elasticity of medical care demand of 0.17 for the $0-25 \%$ co-insurance range and 0.22 for the $25-95 \%$ co-insurance range. To calculate comparable measures, I simulate the within-year decision-making model under three cost-sharing arrangements. All three arrangements feature no deductible, no stop loss, and no premium; differing only by a universal co-insurance rate, which is set to $0 \%$, $25 \%$, or $95 \% .{ }^{62}$ I estimate a co-insurance elasticity of medical care demand of 0.18 for the $0-25 \%$ co-insurance range and 0.28 for the $25-95 \%$ co-insurance range. The remaining three comparisons are straightforward. The measures used by Keeler and Rolph (1988) and Einav et al. (2013) compare expenditure under full insurance and no insurance. The measure used by Bajari et al. (2013) compares expenditure under no insurance and observed/preferred insurance status. ${ }^{63}$

This exercise highlights an important feature of the within-year decision-making model. In principle, the flexibility of the model allows one to calculate other measures of moral hazard effects that are observed in the literature so that comparisons can be drawn. However, despite my efforts, the various moral hazard measures reported in Table 6.8 are not truly comparable. Each of these studies uses a different population of individuals in their empirical analysis. These populations differ in observed features, likely differ in unobserved features, and select health insurance from different sets of alternatives; all of which are likely to impact

\footnotetext{
${ }^{62}$ The RAND HIE plans had no premium and those used for price elasticity estimation had no deductible. The plans did feature a $\$ 1000$ stop loss, but efforts are made by the researchers to avoid the distortions in price elasticity estimates caused by this dynamic incentive for reasons explained in Keeler et al. (1977). Specifically, Manning et al. (1987) "examine demand for episodes of treatment by individuals who are more than $\$ 400$ from their (stop loss) limit. This strategy gives an approximation of the true price effect if such people treat the true probability of exceeding their limit as nearly zero.” Rather than recreating their approximation technique, I eliminate the stop loss in simulation.

${ }^{63}$ Kowalski (2013) also estimates the average increase in expenditure when moved from no insurance to full coverage. She finds an increase of only $\$ 16$ (in 2003 dollars), meaning a comparable moral hazard estimate to Einav et al. (2013) would be less than 1\%. Also, note that Bajari et al. (2013) do not allow for income effects in their estimate, by adjusting the budget constraint in the no-insurance case so that the observed consumption bundle is guaranteed to be affordable. Their estimate would increase without this adjustment.
} 
Table 6.8: Other Measures of the Effects of Moral Hazard

\begin{tabular}{|c|c|c|c|}
\hline & Measure & $\begin{array}{l}\text { Reported } \\
\text { Estimate }\end{array}$ & $\begin{array}{c}\text { Within-Year } \\
\text { Model Estimate }\end{array}$ \\
\hline Manning et al. (1987) ${ }^{\mathrm{a}}$ & $\begin{array}{c}\text { Co-insurance arc elasticity } \\
\text { of medical care demand } \\
0 \% \rightarrow 25 \%, 25 \% \rightarrow 95 \% \text { b }\end{array}$ & $0.17,0.22$ & $0.18,0.28$ \\
\hline Keeler and Rolph (1988) & $\begin{array}{l}\text { Proportion of full insurance } \\
\text { expenditure not explained } \\
\text { by moral hazard }\end{array}$ & $55 \%$ & $49 \%$ \\
\hline Einav et al. (2013) & $\begin{array}{l}\text { Percentage increase in mean } \\
\text { annual expenditure, no } \\
\text { insurance to full coverage }^{\mathrm{d}}\end{array}$ & $30 \%$ & $111 \%$ \\
\hline Bajari et al. (2013) & $\begin{array}{l}\text { Proportion of preferred insur- } \\
\text { ance expenditure explained } \\
\text { by moral hazard }\end{array}$ & $45 \%$ & $48 \%$ \\
\hline
\end{tabular}

a These results are also reported in Keeler and Rolph (1988).

b The arc elasticity is calculated as $E_{\text {arc }}=\left(\left(q_{2}-q_{1}\right) /\left(p_{2}-p_{1}\right)\right) \times\left(\left(p_{2}+p_{1}\right) / 2\right) /\left(\left(q_{2}+q_{1}\right) / 2\right)$, where $q$ is mean annual medical care expenditure and $p$ is the co-insurance rate. Manning et al. (1987) make this calculation for each type of care and then weight elasticities for various types of care by share of spending.

c $q_{2 i} / q_{1 i}$ is calculated for each individual, where $q_{2 i}$ is total annual medical care expenditure with a $95 \%$ co-insurance rate (near no insurance) and $q_{1 i}$ is total annual medical care expenditure with full insurance for individual $i$. The population mean is reported above.

$\mathrm{d}\left(q_{2}-q_{1}\right) / q_{1}$ is calculated, where $q_{2}$ is mean total annual medical care expenditure for the population under full coverage and $q_{1}$ is mean total annual medical care expenditure for the population under no coverage.

e $\left(q_{2 i}-q_{1 i}\right) / q_{2 i}$ is calculated for each individual, where $q_{2 i}$ is total annual medical care expenditure with preferred/chosen coverage and $q_{1 i}$ is total annual medical care expenditure with no coverage for individual $i$. The population mean is reported above.

\section{the estimated effect of moral hazard on medical care consumption. ${ }^{64}$}

Thus, two conclusions can be drawn. First, the comparisons made in Table 6.8 serve to provide some external validity for my findings. The table suggests that the within-year model predicts a level of price sensitivity that is near what has been found in the literature, but due to both observed and unobserved differences in estimation samples, these estimates

\footnotetext{
${ }^{64}$ The RAND HIE data used by Manning et al. (1987) and Keeler and Rolph (1988) include both children and married individuals, while my data do not. Einav et al. (2013) and Bajari et al. (2013) use claims data from a single employer, though not the same employer. In general, employer data feature a more homogenous population and insurance plans are selected from a limited set of health insurance alternatives, which are subsidized by the employer. Analysis is also limited to insured individuals, as claims data are not collected for those declining coverage. Bajari et al. (2013) include all employees selecting single coverage, while Einav et al. (2013) include all employees. Also, individuals in each of these studies face vastly different healthcare environments, as my data were collected from 1996-1999, the RAND HIE data used by Manning et al. (1987) and Keeler and Rolph (1988) were collected from 1974-1980, and the claims data used by Einav et al. (2013) and Bajari et al. (2013) were collected from 2003-2006 and 2002-2004, respectively.
} 
are not directly comparable with those found in the literature. Second, an important objective of this research project is to determine whether or not a model of within-year medical care decision making produces moral hazard effects that are different from an annual expenditure model. One way to answer this question is to compare my estimates to the estimates of Bajari et al. (2013) or Einav et al. (2013), who each estimate structural annual expenditure models. However, again due to differences in estimation samples, this approach would be naive.

In what follows, I perform a series of counterfactual simulations to study how different assumptions imposed on a within-year decision-making model lead to different moral hazard effects. By using the same model and data across simulations, I can isolate the impact that specific assumptions have on estimated moral hazard effects, which is not possible when comparing results across papers. The most important counterfactual imposes on the withinyear decision-making model a set of assumptions that are consistent with the assumptions made implicitly in most annual expenditure models.

\subsubsection{Moral Hazard and Modeling Assumptions}

The assumptions imposed on a behavioral model are likely to impact the estimated effects of moral hazard. For example, if medical care consumed over the course of the insurance year is allowed to improve an individual's health, then simulating behavior under no coverage should produce the following: an immediate reduction in medical care consumption due to higher out-of-pocket costs (price effect), followed by an increase in the occurrence of illness and poor health outcomes, followed by an increase in medical care consumption (health effect), as he attempts to cure the illness and improve his health status. A model that does not allow medical care to improve future illness and health outcomes predicts only the price effect. Therefore, a model that allows medical care to improve health over the course of the year should predict greater medical care consumption in the uninsured state, producing a smaller moral hazard effect. Assumptions concerning an individual's knowledge of current and future price and health outcomes may affect estimated moral hazard effects as well. For 
example, if an individual does not know the total price of medical care prior to consumption, then loss of insurance coverage represents not only an increase in the out-of-pocket cost of medical care but exposure to greater uncertainty as well. ${ }^{65}$ If an individual is risk averse, then his medical care consumption should respond more (negatively) to the loss of insurance coverage when he does not know medical care prices prior to consumption. As a result, a model that assumes medical care prices are unknown prior to consumption should produce larger moral hazard effects.

The within-year decision-making model allows medical care consumption to alter future health outcomes, assumes medical care prices are unknown prior to consumption, and allows for uncertainty about future within-year medical care price and health outcomes. The annual expenditure models of Cardon and Hendel (2001), Einav et al. (2013), and Kowalski (2013) are characterized by a health insurance decision at the beginning of a year, followed by an exogenous annual health shock, which is followed by an annual medical care expenditure decision. ${ }^{66}$ However, an individual represented by the data used in estimation actually makes a series of daily medical care consumption decisions and receives multiple health shocks over an insurance year; thus, a number of assumptions are made implicitly in these models in order to aggregate behavior and outcomes up to an annual level. First, as the annual health outcome is assumed to be exogenously determined (i.e., independent of prior medical care consumption), so too are within-year health outcomes (assumption 1). Second, in order for a daily decision maker to be able to optimally determine his aggregated annual expenditure level, medical care prices must be known at the time of purchase (assumption 2). Third,

\footnotetext{
${ }^{65}$ Health insurance cost-sharing characteristics protect the insured by truncating the right tail of the medical care price distribution. For example, assume an insured individual knows that upon visiting the hospital he faces a total price of $\$ 500$ with probability 0.90 or $\$ 2,000$ with probability 0.10 . Assuming a $\$ 300$ deductible and $\$ 50$ hospital co-pay, an insured individual faces a maximum out-of-pocket cost of $\$ 350$. An uninsured (risk averse) individual is worse off than an insured individual for two reasons: he faces a larger expected out-of-pocket cost (\$650) and receives disutility from exposure to the risk of a high price draw.

${ }^{66}$ Bajari et al. (2013) is similar but does not feature an insurance decision. Also, unlike the other papers listed, Bajari et al. allow for some uncertainty at the time of medical care consumption. The authors assume that an individual selects his total annual medical care expenditure while knowing the distribution of the proportion of that expenditure he must pay out-of-pocket, rather than the exact proportion.
} 
the annual expenditure model assumes that all uncertainty is revealed prior to an expenditure decision. This assumption suggests that all health and price shocks that a daily decision maker receives over the course of an insurance year are known at the beginning of the year (assumption 3).

I use the within-year decision-making model and estimated parameters to calculate the effect of moral hazard under assumption 1 (simulation 1), under assumption 2 (simulation 2), and under assumptions 1, 2, and 3 (simulation 3); where the last set of assumptions is most representative of an annual expenditure model. The moral hazard effect is calculated by simulating the model twice for each set of assumptions: once assuming individuals have no health insurance and once assuming individuals hold health insurance (i.e., their selected plans when forced to hold coverage; from Section 6.3). To impose an assumption of exogenous monthly health transitions (assumption 1) on the within-year decision-making model, I update monthly illness states and general health status using a predetermined set of outcomes, rather than allowing illness and general health probabilities to be altered by medical care consumption. ${ }^{67}$ In simulation, an individual still solves the optimization problem as if medical care is productive as the model's parameters reflect that this is the primary benefit of consuming medical care. To impose an assumption of price certainty prior to medical care consumption (assumption 2) on the within-year decision-making model, an individual receives one price draw for each type of medical care in each month, rather than integrating

\footnotetext{
${ }^{67}$ The predetermined set of illness and general health outcomes is taken from the simulation in Section 6.2 (i.e., simulation under normal conditions). Ideally, observed outcomes from the data would provide the exogenous transitions needed for simulation 2 , as these outcomes would best reflect the assumptions of those modeling annual expenditure decisions under alternative insurance schemes. However, because general health status is not observed in every month in the data, this alternative set of outcomes is used.
} 
over the conditional distribution from which prices are drawn. ${ }^{68}$ Perfect foresight (assumption 3) is imposed on the model by providing an individual with all future price, health, and preference shocks in the first month of the insurance year.

Without altering any assumptions of the within-year decision-making model, health insurance acquisition is predicted to increase mean annual medical care expenditures by $92 \%$. (I refer to this simulation as the preferred simulation.) In simulation 1, assuming that withinyear health transitions are exogenous increases the estimated effect of moral hazard to $100 \%$, which is consistent with discussion above. In the preferred simulation, the probability of acute illness increases by $2.9 \%$ in the first three months (due to lower medical care consumption when uninsured), leading to an increase in medical care consumption throughout the remainder of the year.

In simulation 2, where prices are known prior to consumption, health insurance acquisition is predicted to increase mean annual medical care expenditures by $81 \%$, which is consistent with the discussion above. In this simulation, the decrease in medical care consumption caused by the loss of health insurance is $14.5 \%$ for doctor visits, $44.4 \%$ for hospital days, and $1.7 \%$ for prescription drugs. In the preferred model, the decrease is $20.1 \%$ for doctor visits, $78.0 \%$ for hospital days, and $1.7 \%$ for prescription drugs. If the price paid for medical care were the same in simulation 2 as it is in the preferred simulation, then this consumption pattern would suggest a much larger difference in estimated moral hazard effects. However, because individuals in simulation 2 observe medical care prices, they strategically purchase medical care at lower prices when uninsured. The average price paid for medical care decreases by $7.5 \%$ for doctor visits and $63.8 \%$ for hospital days when individuals lose health

\footnotetext{
${ }^{68}$ In practice, an individual receives one set of 12 price draws for each of the three types of medical care. Each of the 12 price draws corresponds to a specific combination of acute illness state, chronic illness state, and general health status. This strategy ensures that when an individual receives a high price draw he cannot simply wait for a lower price to arrive in the following month. A new set of prices is then drawn if his illness state or general health status changes. Note that in simulation 2, at any given time, an individual only knows the medical care prices that correspond to his current illness state and general health status. He does not know the price that he would face in another wellness state, nor does he know that prices will remain the same in the following month if his wellness state remains unchanged.
} 
insurance in simulation 2 . In the preferred simulation, the decrease is $1.1 \%$ for doctor visits and $39.9 \%$ for hospital days.

In simulation 3, which most closely reflects the assumptions of an annual expenditure model, moral hazard is predicted to increase mean annual medical care expenditure by $74 \%$. This estimate is smaller than the moral hazard effect produced by the preferred simulation $(92 \%)$. The estimate is also closer to the moral hazard effects generated by annual expenditure models in the literature. The change in the price paid for medical care caused by the loss of health insurance in simulation 3 is almost identical to simulation 2 . The root cause of the lower estimated moral hazard effect is that medical care consumption decreases more (in response to the loss of coverage) in simulation 2 than in simulation 3 . The decrease in simulation 3 is $13.7 \%$ for doctor visits, $30.7 \%$ for hospital days, and $1.7 \%$ for prescription drugs. The decrease is greatest in the preferred simulation. In simulation 3, an individual solves a model where medical care is thought to not only improve his illness state in probability, but with certainty. Greater consumption when uninsured results, as some individuals consume more medical care (despite its cost) because knowledge of the illness shock ensures that the medical care will improve their illness state in the following month.

In summary, this research assumes that medical care decisions are made throughout an insurance year under conditions of uncertainty. This uncertainty makes medical care less valuable, as medical care consumption requires an individual to forgo a level of non-medical consumption that is known with certainty, in favor of an uncertain level of non-medical consumption and no guarantee of improved health. Health insurance reduces an individual's exposure to the financial risk that is associated with medical care consumption. When uncertainty at the time of medical care consumption is removed from an individual's optimization problem, medical care becomes more valuable, which leads an individual to consume more. An uninsured individual gains the most from the removal of uncertainty, because prior to removal he is exposed to the greatest level of risk. The burden of uncertainty when uninsured ultimately leads the within-year decision-making model to generate a larger moral hazard 
effect than what has been estimated previously in the literature.

\subsubsection{Moral Hazard and Insurance Status}

Among the many positive features of the MEPS data, the inclusion of both insured and uninsured individuals is of particular use in the study of moral hazard. In the U.S., the insured and uninsured populations have different observed and (likely) unobserved characteristics which may lead to differential responses to health insurance acquisition. Identifying this difference is important because many of the key economic questions relating to moral hazard effects focus on either the insured or uninsured population, but not both. For example, to study the welfare implications of overconsumption caused by moral hazard, one must know how much more medical care the insured population consumes because of their insurance; however, the response of uninsured individuals to coverage is not relevant. Conversely, to predict the increase in medical care consumption that would result from a strict individual health insurance mandate (100\% coverage), one must have an estimate of how much more uninsured individuals consume once they become covered; however, the response of insured individuals to coverage is not relevant. The recent literature that studies moral hazard effects has utilized claims data (Bajari et al. 2013; Einav et al. 2013; Kowalski 2013), which does not include the medical care consumption patterns of uninsured individuals, so moral hazard effects cannot be estimated for that group.

I conduct a series of counterfactual simulations in order to study differences in how the insured and uninsured populations respond to a change in insurance status. First, I allow individuals to select health insurance optimally, as in Section 6.2, which identifies each individual as insured or uninsured. I then simulate behavior for each individual in an uninsured state and in an insured state, where the uninsured group from the first step is forced to select a plan, as in Section 6.3. I then calculate the response to insurance acquisition for both the group that finds it optimal to purchase insurance and the group that finds it optimal not to 
purchase insurance. I find that the insured group increases mean annual medical care expenditure by $96 \%$ ( $\$ 728$ to $\$ 1426$ ) and the uninsured group increases mean annual medical care expenditure by only 55\% (\$366 to $\$ 568$ ). ${ }^{69}$ As expected, the differential response is driven by both observed and unobserved differences between the two sets of individuals. Table 6.9 shows that the uninsured are younger, poorer, more likely to be male, and, in this sample, enter the year in a better illness state. According to Table 6.1 males have significantly lower preferences for doctor visits and prescription drug consumption and Table 6.5 suggests that medical care consumption is lower when individuals are in a better illness state. Also, the uninsured are more likely to be of the unobserved type 2, which is associated with overall wellness and low preferences for medical care.

Table 6.9: Characteristics of the Insured and Uninsured

\begin{tabular}{lrr}
\hline & Insured & Uninsured \\
\hline Observed Characteristics & & \\
male & 0.48 & 0.53 \\
black or hispanic & 0.26 & 0.30 \\
education & 13.7 & 12.95 \\
age & 39.9 & 36.8 \\
MSA & 0.84 & 0.76 \\
income & 34728.3 & 26064.7 \\
acute illness at $t=1$ & 0.38 & 0.32 \\
chronic illness at $t=1$ & 0.38 & 0.27 \\
poor health at $t=1$ & 0.30 & 0.30 \\
good health at $t=1$ & 0.34 & 0.33 \\
excellent health at $t=1$ & 0.36 & 0.37 \\
number of plans available & 5.6 & 2.9 \\
Unobserved Types & & \\
type 1 & 0.09 & 0.01 \\
type 2 & 0.56 & 0.75 \\
type 3 & 0.17 & 0.09 \\
type 4 & 0.18 & 0.15 \\
\hline Simulated Individuals & 10,881 & 1,449 \\
\hline
\end{tabular}

These differences first lead uninsured individuals to purchase less generous plans when

\footnotetext{
${ }^{69}$ Finkelstein et al. (2012) find that previously uninsured individuals in Oregon increase their medical care expenditure by $25 \%$ the first year after gaining access to Medicare; Baicker et al. (2013) find an increase of 35\% in the second year.
} 
forced into coverage (they also choose from less generous alternatives sets). In comparison to the plans selected by the insured population, those wishing to remain uninsured select a plan that has a higher out-of-pocket premium; that is more likely to have a deductible and, conditional on having a deductible, has a larger deductible; that is equally likely to have a stop loss but, conditional on having a stop loss, has a larger stop loss; that has less generous doctor and hospital cost-sharing characteristics; and that is more likely to be a FFS type plan. The differential response to insurance acquisition between the insured and uninsured is driven in part by the less generous plan features that characterize the plans selected by the previously uninsured; however, the uninsured are less responsive to insurance acquisition independent of insurance selection. In another counterfactual simulation, I force all individuals into a common plan and calculate moral hazard effects separately for the insured and uninsured groups. ${ }^{70}$ I find that mean annual medical care expenditure in the insured population increases by $66 \%$; while mean annual medical care expenditure in the uninsured population increases by $47 \%$.

As mentioned above, accounting for differential responses to insurance acquisition can be important for certain policy questions. For example, assume one is interested in how total U.S. medical care expenditure would be affected by a new health insurance policy that required all individuals to purchase coverage. There are several measures of moral hazard effects that could be taken from this research, or outside literature, to predict the such a change in expenditure. ${ }^{71}$ If one assumes that the average uninsured individual responds to coverage just as the average insured individual does, then the policy leads the previously uninsured to increase their medical care expenditure by $96 \%$, which is $\$ 43.2$ billion (in 2011 dollars)

\footnotetext{
${ }^{70}$ The common plan is a fee-for-service plan that has an annual out-of-pocket premium of $\$ 500$, a deductible of $\$ 200$, a stop loss of $\$ 1500$, a doctor visit co-pay of $\$ 10$, a hospital co-insurance rate of $15 \%$, and a prescription drug co-insurance rate of $19 \%$.

${ }^{71}$ The moral hazard effects estimated in this research were not derived with an objective of predicting changes in total U.S. medical care expenditure in response to a change in insurance policy. The estimation sample includes single, childless, employed individuals who get an insurance offer from their employer. However, this exercise exemplifies how even small differences in the estimated effects of moral hazard for different populations can lead to large differences in policy predictions.
} 
of new spending. ${ }^{72}$ This assumption would be required in order to use the estimated moral hazard effects of (Bajari et al. 2013; Einav et al. 2013; Kowalski 2013), who only study insured individuals, to answer this question. If, on the other hand, one assumes that the average uninsured individual responds to coverage just as the average individual (independent of coverage status) does, then the policy leads the previously uninsured to increase their medical care expenditure by $92 \%$, which is $\$ 41.4$ billion (in 2011 dollars) of new spending. However, if one fully account for the differences between the insured and uninsured populations, then the policy leads the previously uninsured to increase their medical care expenditure by $55 \%$, which is $\$ 24.8$ billion (in 2011 dollars) of new spending.

\subsection{Counterfactual Experiment: An Individual Health Insurance Mandate}

Among other things, the 2010 Patient Protection and Affordable Care Act (ACA) requires almost all U.S. citizens to carry a minimal amount of health insurance coverage. ${ }^{73}$ This individual mandate is one of the more controversial pieces of the law. Proponents argue that forcing the (presumably young and healthy) uninsured into the market will help to indemnify the risk pool, subsidizing insurance premiums for the sick. Opponents worry that increased spending, due to moral hazard, will follow the increase in insurance coverage. Thus, in designing the individual mandate, policy makers must consider both the rate at which individuals who are currently uninsured will purchase coverage (for a given penalty) and the increase in medical care expenditure that results from the increase in coverage. The within-year model presented in this dissertation has the capacity to predict both of these outcomes. ${ }^{74}$

\footnotetext{
${ }^{72}$ Average medical care expenditure among the 47 million uninsured U.S. citizens in 2011 was $\$ 958$ (in 2011 dollars, MEPS).

${ }^{73}$ Individuals with incomes below the tax filing threshold (\$9,750 for an individual in 2013) and those who cannot find coverage that costs less than 8 percent of their income are exempt from penalties imposed on the uninsured.

${ }^{74}$ This counterfactual implements the individual mandate provision of the ACA only. There are many other regulations that the ACA will impose upon the marketplace that are not considered. Furthermore, the empirical analysis conducted here focuses on a population of individuals who are unmarried, childless, employed, between the ages of 19 and 64, and have the ability to purchase health insurance through their employers.
} 
Under the ACA, an individual who is currently uninsured and receives an ESHI offer faces the following three alternatives: (1) purchase a plan through his employer, (2) remain uninsured and pay a penalty, or (3) purchase coverage privately (potentially thorough a newly formed health insurance exchange). In this policy simulation, I limit the alternative set to options (1) and (2). ${ }^{75}$ Table 6.10 reports the predicted rates of health insurance take-up for a number of different penalties. Row 1 provides the predicted percent of the population that chooses to remain uninsured, despite receiving an ESHI offer, when there is no penalty for refusing coverage. Rows 2, 3, and 4 mimic the planned penalties that the ACA will enforce in 2014,2015 , and 2016, respectively. The 2016 penalty represents fully implementation of the policy. In 2014, an individual who does not possess the minimal level of health insurance coverage is required to pay a penalty of $\$ 95$ (in 2014 dollars) or $1 \%$ of his income (whichever is larger). ${ }^{76}$ The model predicts that the percentage of uninsured individuals will decrease in the first year from $11.8 \%$ to $9.6 \%$, or that $81.7 \%$ of the previously uninsured individuals will remain uninsured. Rows 4 through 12 report the percent of individuals who remain uninsured given larger flat rate penalties, but holding the percentage of income penalties fixed at the 2016 level of $2.5 \%$.

The table offers two interesting findings. First, the planned penalty for 2016 (i.e., the greater of $\$ 695$ or $2.5 \%$ of income) reduces the percent of uninsured individuals in the population from $11.8 \%$ to $6.0 \%$. The demographic make-up of the uninsured population used

\footnotetext{
${ }^{75}$ Private health insurance is rarely purchased by individuals receiving an ESHI offer in the US because ESHI plans are generally subsidized by employers and receive preferential tax treatment. Health insurance that is provided to employees as part of a compensation package is not taxed by the US government. Plans purchased on the private market are paid for with taxable income. However, some single individuals who receive an ESHI offer will be eligible to enroll in a health insurance exchange and will qualify for tax credits. To purchase health insurance through a newly formed health insurance exchange, an individual's least expensive ESHI option must have an out-of-pocket premium that exceeds $9.8 \%$ of his income or his employer's premium contribution is less than $60 \%$ of the premium. To receive a tax credit, an individual's income must be between 100 and 400 percent of the federal poverty line (about $\$ 11,490$ to $\$ 45,960$ for a single individual in 2013 ).

${ }^{76}$ To discount the penalties to 1996 dollars I assume an inflation rate of $3 \%$ for all years after 2013 and the average annual CPI inflation rate reported by the Bureau of Labor Statistics for years between 1996 and 2013.
} 
Table 6.10: Predicted Health Insurance Coverage Rate

\begin{tabular}{|c|c|c|c|c|}
\hline \multicolumn{3}{|c|}{ Penalty } & \multirow[t]{2}{*}{$\%$ Uninsured } & \multirow[t]{2}{*}{$\%$ Remaining Uninsured } \\
\hline 1996 dollars & 2016 dollars & $\%$ of income & & \\
\hline 0.00 & 0.00 & 0.0 & 11.8 & 100.0 \\
\hline 61.82 & $95.00^{\dagger}$ & 1.0 & 9.6 & 81.7 \\
\hline 205.14 & $325.00^{\ddagger}$ & 2.0 & 7.4 & 63.1 \\
\hline 425.54 & 695.00 & 2.5 & 6.0 & 50.9 \\
\hline 489.83 & 800.00 & 2.5 & 5.8 & 49.3 \\
\hline 612.28 & 1000.00 & 2.5 & 5.1 & 43.3 \\
\hline 734.74 & 1200.00 & 2.5 & 4.4 & 37.9 \\
\hline 918.41 & 1500.00 & 2.5 & 3.7 & 31.8 \\
\hline 1224.56 & 2000.00 & 2.5 & 2.9 & 24.4 \\
\hline 1530.70 & 2500.00 & 2.5 & 2.2 & 18.6 \\
\hline 1836.84 & 3000.00 & 2.5 & 1.6 & 13.5 \\
\hline 2142.98 & 3500.00 & 2.5 & 1.3 & 11.0 \\
\hline 2449.12 & 4000.00 & 2.5 & 1.0 & 8.8 \\
\hline
\end{tabular}

in estimation and simulation make this take-up rate particularly important. The sample includes single, employed individuals, who are likely healthier and wealthier than the average uninsured individual in the population. Therefore, a high take-up rate for this subgroup is necessary for the success of the bill, which requires the healthy to purchase coverage in order to subsidize the premiums of the sick. The proportion of uninsured individuals could be further reduced below 3\% of the population by increasing the flat penalty to $\$ 2000$ (in 2016 dollars). Second, the table highlights the difficulty that policy makers face in producing universal coverage using an incentive-based scheme. As the penalty is raised, those who remain uninsured are the least sensitive to further penalties. For example, increasing the flat penalty from $\$ 1000$ to $\$ 1500$ (in 2016 dollars) decreases the percentage of uninsured individuals by 1.4 percentage points. As an additional $\$ 500$ is added to the penalty repeatedly, the percentage of uninsured individuals decreases but at a decreasing rate: $0.8,0.7,0.6,0.3$, etc. If the goal is universal coverage, then an alternative policy of forced take-up (implemented by employers, who could be required to enroll employees in a default health insurance plan) may be preferable, but is not without consequence. Using the model and estimated parameters, I 
calculate the average expected welfare loss of among uninsured individuals from this policy to be $\$ 1608$ (2016 dollars). ${ }^{77}$ Note that this penalty is more than two times the fixed dollar penalty at full implementation.

I am also able to examine how total annual medical care expenditure responds to an individual mandate. Assuming premiums and medical care prices are unchanged, spending among previously insured individuals remains unchanged. Among previously uninsured individuals, mean annual medical care expenditure for the newly insured increases by $77 \%$ (moral hazard effect), while expenditure for those remaining uninsured falls by $2.4 \%$ (income/penalty effect). The net effect on the population as a whole is a $1.3 \%$ increase in medical care expenditure. This effect is small for several reasons. First, only $11.8 \%$ of the population is uninsured prior to the policy and are at all affected (under the assumption that medical care prices and insurance premiums are unaffected by the policy). Second, only half of these individuals acquire coverage and increase their spending. Third, the absolute level of mean spending among the newly insured is small relative to those previously insured $(\$ 637$ vs. \$1448, respectively).

\footnotetext{
${ }^{77}$ The average expected welfare loss is measured as the average penalty that would make an uninsured individual indifferent between remaining uninsured and paying the penalty or being insured and paying the premium.
} 


\section{CONCLUSION}

In this dissertation, I study insurance-induced moral hazard using a dynamic model of within-year medical care consumption. An individual's optimization problem is defined by an annual health insurance decision, followed by a sequence of monthly medical care consumption decisions made over the course of a health insurance year. The within-year dynamic structure stands in contrast to existing work that examines the relationship between health insurance and medical care demand by aggregating medical care decisions up to an annual level. The disaggregated model of decision making more accurately describes the data generating process by allowing medical care consumption to alter future health outcomes, assuming medical care prices are unknown prior to consumption, and allowing for uncertainty about future within-year medical care price and health outcomes.

The effect of moral hazard on total annual medical care expenditure is calculated by simulating individuals' behavior under various insurance conditions. There are two main findings. First, the within-year model produces a moral hazard effect that is $24 \%$ larger than an alternative model that imposes the more restrictive assumptions of a typical annual expenditure model. Ultimately, the presence of uncertainty at the time of medical care consumption in the within-year decision-making model decreases the expected value of medical care consumption. The larger moral hazard effect is driven by low medical care consumption when

uninsured, as risk averse individuals who face uncertainty are exposed to significant risk in consumption. Second, I find heterogeneous moral hazard effects across the population. Of importance to policy makers is that insured individuals are found to be much more responsive to coverage than uninsured individuals $(96 \%$ vs. $55 \%$ increase in spending in response to coverage, respectively). 
This research advances the economic literature on insurance-induced moral hazard by allowing for dynamic within-year incentives and uncertainty in a model of health insurance and medical care consumption decisions; however, this research could also benefit from additional data that would allow for the modeling of additional supply and demand side outcomes/behaviors. Supplementing the medical care consumption information that I observe with data on recommended courses of treatment for illnesses would allow one to disentangle the roles of doctors and patients in the medical care decision-making process. Furthermore, observing all employees within a firm (i.e., risk pools) may allow for insurance premiums to be determined in equilibrium, which would allow one to study how new insurance alternative sets affect insurance take-up, premiums, and medical care expenditure. These extensions are interesting both theoretically and empirically and are left for future work. 


\section{A APPENDIX: CHARTS AND TABLES}

Table A.1: Representativeness of the Sample

\begin{tabular}{lrrrrr}
\hline & \multicolumn{2}{c}{ Whole Sample } & & \multicolumn{2}{c}{ Estimation Sample } \\
\cline { 2 - 3 } \cline { 5 - 6 } & mean & s.d. & & mean & s.d. \\
\hline age & 36.88 & 12.21 & & 39.56 & 11.70 \\
education (highest grade completed) & 13.40 & 2.53 & 13.62 & 2.46 \\
income (in 1996 dollars) & 30985.62 & 21432.55 & 33713.84 & 21953.46 \\
male & 0.53 & $*$ & 0.48 & $*$ \\
lives in a MSA & 0.84 & $*$ & 0.83 & $*$ \\
Hispanic & 0.14 & $*$ & 0.12 & $*$ \\
black & 0.16 & $*$ & 0.14 & $*$ \\
federal employee & 0.03 & $*$ & 0.09 & $*$ \\
northeast & 0.19 & $*$ & 0.19 & $*$ \\
midwest & 0.24 & $*$ & 0.25 & $*$ \\
south & 0.35 & $*$ & 0.34 & $*$ \\
west & 0.22 & $*$ & 0.22 & $*$ \\
excellent health status & 0.34 & $*$ & 0.32 & $*$ \\
good health status & 0.35 & $*$ & 0.35 & $*$ \\
poor health status & 0.31 & $*$ & 0.33 & $*$ \\
total annual expenditure $\dagger$ & 1490.37 & 4033.53 & 1636.53 & 3063.95 \\
insured all year & 0.81 & $*$ & 0.91 & $*$ \\
\hline Sample Size & 4859 & & 1232 & \\
\hline
\end{tabular}

$\dagger$ This expenditure level was take directly from the MEPS data and includes types of medical care spending not included in this analysis (e.g., dental and eye care, home healthcare, medical equipment, etc.). 
Table A.2: Structural Price Parameter Estimates

\begin{tabular}{|c|c|c|c|c|c|c|}
\hline & \multicolumn{2}{|c|}{ Doctor Price } & \multicolumn{2}{|c|}{ Hospital Price } & \multicolumn{2}{|c|}{ Prescription Price } \\
\hline & est. & s.e. & est. & s.e. & est. & s.e. \\
\hline \multicolumn{7}{|l|}{ Price } \\
\hline constant & 4.2338 & 0.0695 & 6.0503 & 0.0315 & 4.4014 & 0.0608 \\
\hline male & 0.0243 & 0.0189 & 0.0508 & 0.0537 & -0.2485 & 0.0169 \\
\hline non-white (black or Hispanic) & -0.1183 & 0.0357 & 0.0061 & 0.0541 & -0.0316 & 0.0155 \\
\hline education (highest grade completed) & -0.0338 & 0.0051 & -0.0031 & 0.0165 & 0.0115 & 0.0035 \\
\hline age & 0.0015 & 0.0011 & 0.0089 & 0.0016 & -0.0001 & 0.0006 \\
\hline lives in a MSA & 0.1207 & 0.0299 & 0.1294 & 0.1229 & -0.0819 & 0.0173 \\
\hline income (in 1996 dollars) & 0.0033 & 0.0006 & 0.0079 & 0.0041 & 0.0013 & 0.0004 \\
\hline March/April (indicator) & 0.1841 & 0.0464 & 0.3457 & 0.1476 & -0.0566 & 0.0329 \\
\hline May/June (indicator) & 0.2524 & 0.0475 & 0.3244 & 0.1317 & -0.1490 & 0.0329 \\
\hline July/August (indicator) & 0.1513 & 0.0449 & 0.0075 & 0.0922 & -0.1877 & 0.0340 \\
\hline September/October (indicator) & 0.1204 & 0.0437 & 0.5808 & 0.1897 & -0.1458 & 0.0327 \\
\hline November/December (indicator) & 0.1330 & 0.0487 & 0.2732 & 0.1787 & -0.0339 & 0.0251 \\
\hline HMO & -0.0856 & 0.0273 & 0.3182 & 0.1366 & -0.1341 & 0.0149 \\
\hline PPO & -0.0195 & 0.0206 & -0.0017 & 0.0205 & 0.0916 & 0.0258 \\
\hline no insurance & 0.1237 & 0.0501 & -0.7416 & 0.1593 & -0.2526 & 0.0339 \\
\hline acute illness & 0.0398 & 0.0254 & -0.1495 & 0.1035 & 0.1290 & 0.0166 \\
\hline chronic illness & 0.0072 & 0.0282 & -0.2108 & 0.1289 & 0.2459 & 0.0220 \\
\hline less than excellent health status & 0.4736 & 0.0480 & -0.0130 & 0.0163 & -0.1186 & 0.0356 \\
\hline less than good health status & -0.3800 & 0.0311 & 0.8883 & 0.1240 & 0.2674 & 0.0263 \\
\hline gamma shape parameter & 1.2102 & 0.0204 & 0.5761 & 0.0371 & 2.0456 & 0.0226 \\
\hline
\end{tabular}


Table A.3: Initial Condition Probability Parameter Estimates

\begin{tabular}{|c|c|c|c|c|c|c|}
\hline & \multicolumn{2}{|c|}{ Health Status } & \multicolumn{2}{|c|}{ Acute Illness } & \multicolumn{2}{|c|}{ Chronic Illness } \\
\hline & est. & s.e. & est. & s.e. & est. & s.e. \\
\hline \multicolumn{7}{|l|}{ Initial Condition } \\
\hline constant & -1.1315 & 0.5323 & -0.1575 & 0.7045 & -2.4117 & 0.8227 \\
\hline male & 0.3068 & 0.1369 & -0.7126 & 0.1713 & -0.5525 & 0.1859 \\
\hline non-white (black or Hispanic) & -0.2747 & 0.1444 & -0.4252 & 0.1814 & -0.2691 & 0.2125 \\
\hline education (highest grade completed) & 0.0795 & 0.0292 & 0.0093 & 0.0374 & 0.0854 & 0.0428 \\
\hline age & -0.0204 & 0.0060 & 0.0032 & 0.0123 & 0.0585 & 0.0139 \\
\hline lives in a MSA & 0.1176 & 0.1681 & 0.0568 & 0.2023 & -0.1542 & 0.2340 \\
\hline income (in 1996 dollars) & 0.0086 & 0.0043 & 0.0007 & 0.0053 & 0.0001 & 0.0055 \\
\hline March/April (indicator) & 0.0860 & 0.2652 & -0.5090 & 0.3144 & $*$ & $*$ \\
\hline May/June (indicator) & -0.0447 & 0.1978 & -0.1933 & 0.3487 & * & $*$ \\
\hline July/August (indicator) & -0.0163 & 0.2538 & 0.0248 & 0.2183 & $*$ & $*$ \\
\hline September/October (indicator) & -0.0756 & 0.2683 & -0.4642 & 0.3147 & * & $*$ \\
\hline November/December (indicator) & $*$ & $*$ & $*$ & $*$ & $*$ & $*$ \\
\hline less than excellent health status & $*$ & $*$ & 0.3630 & 0.3722 & 0.1831 & 0.4490 \\
\hline less than good health status & $*$ & $*$ & 0.1765 & 0.3985 & 0.5640 & 0.5103 \\
\hline less than excellent health status*age & $*$ & * & 0.0014 & 0.0157 & 0.0137 & 0.0195 \\
\hline less than good health status*age & $*$ & $*$ & -0.0019 & 0.0159 & -0.0053 & 0.0207 \\
\hline last year income & 0.0459 & 0.0423 & 0.0167 & 0.0528 & 0.0569 & 0.0590 \\
\hline last year income missing & -0.0378 & 0.2854 & -0.0395 & 0.3608 & -0.0130 & 0.4043 \\
\hline veteran (indicator) & -0.1452 & 0.2242 & 0.2579 & 0.2765 & -0.3692 & 0.3200 \\
\hline foreign born (indicator) & 0.0406 & 0.2272 & 0.3385 & 0.2622 & -0.6485 & 0.3189 \\
\hline cut-point & 1.5432 & 0.0791 & * & * & $*$ & * \\
\hline
\end{tabular}


Table A.4: Closing Function Structural Parameter Estimates

\begin{tabular}{|c|c|c|c|}
\hline & Parameter & Estimate & $\mathrm{SE}$ \\
\hline \multicolumn{4}{|l|}{ Closing Funtion } \\
\hline doctor visits & $\gamma_{0}$ & -0.1180 & 0.1220 \\
\hline hospital days & $\gamma_{1}$ & 0.4559 & 0.3368 \\
\hline Rx consumption & $\gamma_{2}$ & -0.4420 & 0.2912 \\
\hline doctor visits $^{2}$ & $\gamma_{3}$ & 0.0154 & 0.0130 \\
\hline hospital days ${ }^{2}$ & $\gamma_{4}$ & -0.0102 & 0.0105 \\
\hline doctor visits*Rx consumption & $\gamma_{5}$ & 0.0265 & 0.0391 \\
\hline doctor visits*hospital days & $\gamma_{6}$ & -0.1135 & 0.0812 \\
\hline hospital days $* \mathrm{Rx}$ consumption & $\gamma_{7}$ & -0.0032 & 0.0369 \\
\hline doctor visits*age & $\gamma_{8}$ & 0.0012 & 0.0042 \\
\hline hospital days*age & $\gamma_{9}$ & -0.0366 & 0.0161 \\
\hline Rx consumption*age & $\gamma_{10}$ & 0.0101 & 0.0131 \\
\hline acute illness & $\gamma_{11}$ & -30.4658 & 6.7544 \\
\hline chronic illness ${ }^{\dagger}$ & $\gamma_{12}$ & -200.0000 & $*$ \\
\hline less than excellent health status & $\gamma_{13}$ & -57.5097 & 7.6595 \\
\hline less than good health status & $\gamma_{14}$ & -64.9785 & 7.4035 \\
\hline
\end{tabular}




\section{B APPENDIX: DATA OVERVIEW}

The empirical exercises included in this research use data from the first four cohorts (1996-1999) of the Medical Care Expenditure Panel Survey (MEPS), which is collected by the Agency for Healthcare Research and Quality (AHRQ). The within-year model requires the following information for each individual in the estimation sample: a set of employersponsored health insurance (ESHI) plans (this includes the cost-sharing features of every plan), an insurance decision, a general set of demographic variables, and two-years of medical care consumption decisions, medical care prices, and health/illness identifiers. Most of these variables cannot be taken directly from the survey, but must be constructed from a set of responses. Furthermore, as is with any large longitudinal data set, many individuals are missing pieces of information because they skipped an interview or failed to answer a particular survey question. In some studies this missing variable problem can be handled by simply dropping individuals from analysis, but with an already limited sample dropping more individuals due to missing variables is not possible here. Therefore, this section serves two purposes. First, it details the construction of variables used in estimation from the raw data. Second, it explains the various data cleaning methods employed to strategically fill missing variable values for the individuals in the estimation sample.

\section{B.1 Demographic Variables}

Individuals are first described by the following demographic variables, which are assumed to be exogenous and time-invariant in estimation: sex, age, education (years of school completed), income, race (categorized as white or non-white), and MSA (categorized as living in an MSA or not). Sample inclusion requires that individuals are employed and single (not married and without children). Also, exclusion restrictions are needed to estimate endogenous initial conditions; these are taken from the previous years National Health Interview Survey (henceforth NHIS, which is the survey from which MEPS participants are drawn) and 
include: prior year income, veteran status, and country of birth (categorized as foreign born or not).

Sex, age, race, employment, and MSA status can be taken directly from the data without explanation. Education is taken from the first interview. Later interviews are used if the first interview response is missing. ${ }^{78}$ Income is the sum of post-tax income, sale earnings, and tax refund (ttlpnx, salepnx, refdpnx). AHRQ provide a cleaned version of these variables with imputations. Individuals self-identify as married (marry31x) and I assume separated individuals are not married. ${ }^{79}$ Parents are identified by other persons living in the household (who are under 19 if non-student or under 24 if student), who identify them as mom or dad (mopid31x, dapid31x).

Veteran Status can be extracted directly from NHIS survey data in 1995 and 1996 (corresponding to 1996 and 1997 MEPS participants). In 1997 and 1998, a veteran question is not asked directly but can be inferred from the question "have you ever been honorably discharged" (miltryds) and from VA medical care coverage information (hikindf, hikindg) ${ }^{80}$ Foreign born status can be taken directly from the data in each year. Previous year income is measured on a 1-8 scale, as shown on the following page.

Income is missing for $33 \%$ of the NHIS population, so I use a missing indicator in estimation. Furthermore, of the 1233 individuals in the estimation sample, only 1179 can be linked to NHIS. Those not matched are new members or previously absent members of NHIS participating households. Of those linked, only 3 are missing foreign born status and 11 are missing veteran status. These individuals and those not linked, are assumed to be native born, non-veterans.

\footnotetext{
${ }^{78}$ Two individuals never report an exact number of years of education; however, they report some college so 14 years of education is assumed.

${ }^{79}$ The marriage condition is meant to eliminate individuals considering family insurance coverage. Separated individuals are considered not married because (I assume) they make individual insurance decisions. If any of these separated individuals hold family coverage then they are dropped from the sample.

${ }^{80} \mathrm{An}$ individual is assumed to be a veteran if miltrydc $=1$ OR hikindf $=1 \&$ age $>18 \&$ male $=1$ OR hikindg $=1 \&$ age $>18 \&$ male $=1$.
} 


$\begin{array}{ll}1 & 0-5 \mathrm{k} \\ 2 & 5-10 \mathrm{k} \\ 3 & 10-15 \mathrm{k} \\ 4 & 15-20 \mathrm{k} \\ 5 & 20-25 \mathrm{k} \\ 6 & 25-35 \mathrm{k} \\ 7 & 35-45 \mathrm{k} \\ 8 & 45 \mathrm{k}+\end{array}$

\section{B.2 Insurance Offer Set}

A requirement for sample inclusion is that individuals are offered ESHI and that they choose to be continuously insured (by an ESHI plan with no switching and no other coverage) or uninsured over a 12 month period. Evidence of an insurance offer can be found in both the employment section (offer31x) and insurance section (hpemmyy). When these variables contradict, I assume the individual receives an offer as they are later removed if the offer is not observed in the IC-Link file. Monthly insurance status (insured or not) can be determined in the main $\mathrm{HC}$ data file (insmmyy). A series of insurance provider type variables in the HC file ensures that the individual is not covered by an additional non-ESHI plan. Information in the Person-Round-Plan file identifies individuals switching ESHI plans during the insurance year and those holding multiple ESHI plans.

In the model, an individual selects a health insurance plan from a set of options offered by his employer. Estimation requires data describing the premium and cost-sharing features of both accepted and offered plans. The fact that MEPS collects such data is one of the two main reasons that it is used in this research. ${ }^{81}$ From 1996-1999 and again in 2001, information about an employee's insurance offer set was gathered from his employer following the first interview. ${ }^{82}$ This insurance information is contained in the confidential MEPS IC file.

\footnotetext{
${ }^{81}$ The other critical characteristic of the MEPS data is that it provides information about health and medical care consumption within the insurance year.

${ }^{82}$ There was one significant change to the collection process that took place after 1996. The 1996 MEPS asked
} 
Using a Census Research Data Center (RDC), the IC file was linked with public individual level data (MEPS HC file) to form the file used in estimation. Unfortunately, about $56 \%$ of the individuals in the $\mathrm{HC}$ file meeting sample inclusion criteria for this research did not have plans included in the IC File and were therefore dropped from the estimation sample. ${ }^{83}$ Among the individuals remaining, many have insurance offer sets featuring plans with missing characteristics. At least 1 of the 12 insurance cost-sharing features is missing in $47 \%$ of the plans observed in the data. The remainder of this section discusses the strategies I use fill these missing values.

Before describing these imputation strategies, it is important to understand that variation in the structure of health insurance plans (specifically, variation in the use of cost-sharing features) is the most significant contributor to missing plan features. For example, some plans simply do not have a deductible, which is the same as having a zero deductible. In completing the survey, some firms skipped the deductible question when the plan did not have a deductible, rather than filling in a zero value. As such, the data often cannot distinguish between a skipped question and a plan without a particular cost-sharing feature. ${ }^{84}$

\section{B.2.1 Logical Imputation}

I begin this exercise with a series of logical imputations which are informed by the observed features of an insurance plan.

1. If no deductible is listed and it is stated that the plan has no deductible, then a zero

each employer specific questions regarding their participating employee. This method caused many employees to refuse to provide their employers information as employees wished to remain anonymous. The method also troubled employers because it was much more difficult to provide information about a particular employee than employees in general (legalities also made employers weary of providing employee specific information). Therefore, from 1997 on, the collection process was altered such that employers were only asked general information about their insurance offerings. AHRQ then used a matching procedure to identify which offered plan was reportedly chosen by employees. Note also that my analysis does not use year 2001 because data collector did not gather information on the month a plan year started, which is critical when taking the data to the model.

${ }^{83}$ See Section 4 and Table 4.1 for a discussion on potential bias introduced by this exclusion.

${ }^{84}$ There is actually a separate question asking whether or not the plan had a deductible, but many plans are missing this information as well. Therefore, I use the "does this plan have a deductible" information when possible, but it cannot be consistently relied upon. 
deductible is coded. If it is indicated that the plan has a deductible, but no deductible value is recorded, then I note this information and use it later. If the response to the "does this plan have a deductible" question is missing then the deductible value is left missing until later.

2. There are three hospital coverage variables (co-insurance, daily co-pay, and per-stay co-pay). If any of these three is given a positive value, then I assume the others (if missing values) do not apply.

3. There are two doctor coverage variables (co-insurance and co-pay). If either of these is given a positive value, then I assume the other (if missing values) does not apply.

4. If a hospital co-pay value is recorded, but per-day/per-stay is not stated, then I assume co-pay greater than 100 is per-stay and less than 100 is per-day.

5. If the stop loss is coded as zero (meaning comprehensive coverage), but the plan features some cost-sharing, then I recode the stop loss to be missing.

6. If hospital and doctor care is free after the deductible is crossed then I set the stop loss equal to the stated deductible.

The scope of the missing information problem after these assumptions can be observed below. I list the number of plans with any missing piece of information by whether or not the plan is held. Plans are flagged if missing out-of-pocket premium, deductible, hospital coverage, doctor coverage, or stop loss. Total premium and plan type (HMO, PPO, or FFS) are never missing in the data. Also, note that for many of these missing characteristics I can determine whether or not the plan characteristic exists (e.g., that the plan has a deductible), just not what the true value is.

\begin{tabular}{|l|c|c|c|}
\hline & Declined Plan & Held Plan & Total \\
\hline No missing features & 2,495 & 434 & 2,929 \\
\hline Missing features & 1,958 & 706 & 2,664 \\
\hline Total & 4,453 & 1,140 & 5,593 \\
\hline
\end{tabular}

Next, I use observed expenditure information in the data to infer missing cost-sharing features of held plans. MEPS collects not only charge and total expenditure data, but also 
out-of-pocket and insurer payment for each medical care episode. This information can be used at times to determine deductible levels and doctor/hospital cost-sharing arrangements for held plans only. For example: If a plan begins in January and every trip to the doctor's office over the course of the year cost the individual \$5 out-of-pocket, then I can impute that the individual had no deductible and a $\$ 5$ co-pay at the doctor. In the spirit of full disclosure, imputation is rarely this easy for a number of reasons; many plans have different cost-sharing characteristics in and out of network, people may not recall the price paid out-of-pocket, and some individuals simply do not pay their medical bills. As such, some subjectivity is necessary to execute this procedure.

I also use this step to exclude some individuals and plans from the final sample. First, I eliminate individuals with large amounts of medical care spending information that cannot possibly be generated under their current coverage. Second, I remove redundant plans, or plans within a specific individuals offer set that are exactly the same according to the model's characterization of insurance or differ only by start month. After removing these redundant plans and making the imputations described above, the number of plans with missing characteristics is as follows:

\begin{tabular}{|l|c|c|c|}
\hline & Declined Plan & Held Plan & Total \\
\hline No missing features & 2,398 & 485 & 2,883 \\
\hline Missing features & 1,847 & 641 & 2,488 \\
\hline Total & 4,245 & 1,126 & 5,371 \\
\hline
\end{tabular}

\section{B.2.2 Matching Method}

After the logical edits described above, I turn to more traditional imputation techniques. I first use a matching procedure that fills in missing insurance characteristics with those of plans matching on observable characteristics. To execute this procedure, I first order all 7028 plans in the original IC-Link file by total premium and network type. I then categorize each plan by its missing characteristics (e.g., missing nothing, missing deductible only, missing deductible and doctor coverage, etc.). Beginning with the plans missing the fewest characteristics, I fill 
in missing information using values from similar plans where the variable is observed. There are 6 rounds of matching, where the level of "similarity" required for a match is relaxed further in each round. The table below describes the bounds on an acceptable match in each round.

\begin{tabular}{|l|c|c|c|c|c|c|}
\hline & \multicolumn{7}{|c|}{ Round } \\
\hline & 1 & 2 & 3 & 4 & 5 & 6 \\
\hline Total premium & 0 & 0 & 25 & 25 & 50 & 50 \\
\hline Total deductible & 0 & 0 & 25 & 25 & 50 & 50 \\
\hline Doctor deductible & 0 & 0 & 25 & 25 & 50 & 50 \\
\hline Hospital deductible & 0 & 0 & 25 & 25 & 50 & 50 \\
\hline Doctor co-pay & 0 & 0 & 5 & 5 & 10 & 10 \\
\hline Doctor co-insurance & 0 & 0 & 5 & 5 & 10 & 10 \\
\hline Hospital co-insurance & 0 & 0 & 5 & 5 & 10 & 10 \\
\hline Hospital daily co-pay & 0 & 0 & 10 & 10 & 20 & 20 \\
\hline Hospital stay co-pay & 0 & 0 & 25 & 25 & 50 & 50 \\
\hline Stop loss & 0 & 0 & 150 & 150 & 300 & 300 \\
\hline Plan type & 0 & 0 & 0 & 0 & 0 & 0 \\
\hline
\end{tabular}

In order to match, every characteristics of the matched plan must fall within the appropriate characteristic bounds centered at the observed characteristics of the plan with some piece of missing information. For example: assume plan A is missing all deductible information, but has values for all other plan characteristics. Further, assume these characteristics are as follows:

- Total premium: 500

- Doctor co-pay: 10

- Doctor co-insurance: none

- Hospital co-insurance: 10

- Hospital co-pay (day): none

- Hospital co-pay (stay): none

- Stop loss: 1500

- Plan type: HMO 
In the first two search rounds, plan A would only be matched if it finds a plan with the exact same characteristics described above. ${ }^{85}$ If a match is found then deductible, hospital deductible, and doctor deductible information from the matched plan are recorded for plan A. In the third and fourth rounds, plan $\mathrm{A}$ is matched if it can find a plan with characteristics fitting in the following bounds: ${ }^{86}$

- Total premium: $[475,525]$

- Doctor co-pay: $[5,15]$

- Doctor co-insurance: $[-5,5]$

- Hospital co-insurance: $[5,15]$

- Hospital co-pay (day): $[-10,10]$

- Hospital co-pay (stay): $[-25,25]$

- Stop loss: [1350, 1650]

- Plan type: HMO

In each round all plans with missing information look for a match before moving on to the next round. A summary of missing plans after the matching procedure can be seen below.

\begin{tabular}{|l|c|c|c|}
\hline & Declined Plan & Held Plan & Total \\
\hline No missing features & 3,973 & 924 & 4,897 \\
\hline Missing features & 272 & 202 & 474 \\
\hline Total & 4,245 & 1,126 & 5,371 \\
\hline
\end{tabular}

\footnotetext{
${ }^{85}$ Only plans with no missing information are available for match. After the first round of matching, some plans previously ineligible for match become eligible, which is why there are two rounds of matching for each set of bounds.

${ }^{86}$ There are several notes to be made here. First, in every round, the plan types must match exactly. Second, the out-of-pocket premium is not used as a criteria for matching because it varies across employers, meaning it has little to do with the commonality of plans in the marketplace. Third, in many instances, it can be assumed with certainty (using other variables in the data) that a plan with a missing deductible (or stop loss) does in fact have a non-zero deductible. In these cases, the match plan must have a non-zero deductible, in addition to the restrictions described above.
} 


\section{B.2.3 Regression Method}

After using this matching technique, there are still 474 plans with missing information. However, the existence of missing information here does not necessarily imply that the observable characteristics of these plans are outside the bounds of normalcy. Because the matching procedure requires similarity in every observable characteristic, it is possible that a plan missing only 1 characteristic, with all other observed characteristics at the sample mean, may not find a match simply because that combination of features is not seen in another plan.

I turn to regression methods to determine missing values for the remaining plans. To reduce the number of regressions required, I assume that all remaining plans are defined by the most popular insurance structure, meaning doctor and hospital specific deductibles, hospital co-pay, and doctor co-insurance are ruled out. I begin by estimating whether or not a plan has a stop loss. ${ }^{87}$ The regression includes all plans reporting a stop loss and those reporting that the plan has no stop loss. These parameters are used to predict the presence of a stop loss for plans where it cannot be determined if a stop loss exists. I then use an OLS regression of stop loss on other plan and company characteristics to predict a stop loss for those known (either through the data or through the previously described prediction) to have one, but no recorded value. ${ }^{88}$ The same procedure is followed for the deductible; a logit for any deductible and an OLS regression to predict the level. Hospital co-insurance, doctor's office co-pay, and out-of-pocket premium equations are all estimating using Tobit models and missing values are replaced with appropriate predictions.

\footnotetext{
${ }^{87}$ For this regression and all other dichotomous outcomes I estimate logit parameters.

${ }^{88}$ Each regression uses the following set of explanatory variables: total premium, has deductible, deductible, has stop loss, stop loss, hospital cost-sharing generosity, doctor's office cost-sharing generosity, firm size, number of firm enrollees, federal plan indicator, year dummies, HMO, and PPO. I also use a number of missing variable dummies that change across equations as information is filled in. Some variables must be removed from this set so that they are not on the right and left hand side of the regression (e.g., stop loss is not included on the right hand side of the stop loss regression).
} 


\section{B.3 Medical Care Consumption}

Survey participants report all medical care consumption that takes place during an interview period. To the best of their ability, individuals provide the date and location of consumption, illness treated, procedure performed, price charged, and the cost-sharing arrangement with their insurance company. Given the length of time covered by an interview period and the number of details associated with any consumption episode, the ability of individuals to recall this information is questionable. To counteract the recall problem, survey administrators contacted all reported medical facilities to verify as much information as possible. ${ }^{89}$ For each medical care type, AHRQ provides both imputed (File 1) and non-imputed (File 2) data, where the former contains episode level consumption variables that combine individual and provider responses. I use File 1 in my analysis. Note that contacting providers increases the reliability of all variables associated with particular visit, but individuals are relied upon entirely to report visits. If they fail to report a particular visit, no medical provider is contacted to verify their absence. The information below describes how I categorize reported medical care consumption into specific types, how consumption units are priced, and how prescription drug consumption dates are determined.

\section{B.3.1 Medical Care Types and Pricing}

MEPS classifies all medical care consumption into inpatient, outpatient, ER, office-based, and prescription drug. For the purposes of this research, inpatient, outpatient, and ER visits are all classified as hospital care. A single trip to the ER or single outpatient visit is equivalent (in terms of consumption decision and price draw) to one day of an inpatient stay. An inpatient stay of 5 days constitutes 5 separate decisions to consume medical care. Doctor visits are

\footnotetext{
${ }^{89}$ Contact with medical providers was made via telephone interview and mailed survey materials. Providers were contacted for every consumption episode, except office-based visits. AHRQ only contacted office providers if (1) the patient received Medicaid, (2) the household held a managed care plan and was selected as part of a $75 \%$ random sample, or (3) the household did not hold an managed care plan or Medicare, but was selected as part of a $25 \%$ random sample.
} 
straight-forward and prescription drug consumption is discussed at length in Section 4.3.

Medical care prices are observed in the data only when individual's consume care. Therefore, an individual only receives a price contribution to his likelihood function in months when he chooses to consume. ${ }^{90}$ The medical care price information needed for the likelihood contribution are taken directly from the data. The total price paid for medical care (insurer payment plus insured payment) is used as the total price. If an individual consumes multiple units of the same type of medical care in a month then the average price paid for that month is used, as each individual can only have one price contribution per month.

There are two issues with the medical care pricing data that must be addressed. The first issue is bundle pricing. At times, rather than pricing individual doctor visits or hospital days, a price is set for a fixed number of visits or for general treatment of an ailment. This pricing strategy is rarely seen in the data (283 of the 13,819 visits reported in the data are priced as part of a bundle) but must be dealt with. I assume that all visits in the bundle are chosen independently. If all visits in the bundle are of the same type (doctor or hospital) then I assume that the price is spread out equally over each visit. If the consumption bundle consists of both hospital days and doctor visits, then an 8/1 price ratio is assumed between hospital and doctor prices, which is consistent with the price ratio observed among non-bundled visits.

The second issue is with observed medical care prices for the uninsured. It should be the case that the list (charged) price is equal to the transaction (paid) price for all uninsured individuals, but this is not the case. (See Section 3.4 for terminology.) At times, the uninsured negotiate directly with their doctors for lower payments or simply fail to pay a medical bill, which is observed as zero payment in the data. If I were to use the payment amount as the total price for medical care (for the uninsured), then the uninsured would face lower

\footnotetext{
${ }^{90}$ This data feature is similar to the labor literature, where wages are only observed for individuals who choose to work. In the labor literature, an employment decision is included in estimation to control for the endogeneity of wages (those who are likely to earn high wages are also likely to work). The model presented in this dissertation controls for the endogeneity of prices (those who are likely to receive low price draws are also likely to consume) by modeling a consumption decision and be allowing for permanent unobserved correlation between the medical care preferences and price draws.
} 
average medical care prices than insured individuals. Left unchanged, these low prices would incorrectly incentivize zero coverage in simulation. In theory, the model should include negotiation costs, medical care quality, and individual credit-scores, which would capture the costs associated with the low payments of the uninsured; however, this would significantly complicate the model. Instead, I use the list (charged) price as the total price for the uninsured in estimation.

\section{B.3.2 Consumption Dates}

The dates of doctor visits and hospital days are consistently reported in the data (roughly $0.1 \%$ of visits are not accompanied by a date). For the few doctor visits and hospital days that are missing a date, a month is selected at random from the appropriate interview period. The dates of prescription drug consumption are much more difficult to extract from the data. The model requires that I observe (in each month) whether or not any prescription drugs are consumed and total drug spending. The goal is then to take from the data the beginning and ending consumption months for each prescription, as well as the total amount spent on each prescription over all consumption months. This total is divided evenly over the consumption months. The prescription price each month used in the model is then the sum of all prescription expenditures within the month.

The first month that a particular prescription is filled can be found in the data for most drugs $(73 \%)$. For those missing a date, I match the prescriptions to illnesses and medical care consumption in the same interview period using ICD-9-CM codes. ${ }^{91}$ By assuming the prescription drug is first taken in the month that the illness began or in the same month as medical care consumption I can impute some prescription drug beginning dates $(85 \%$ have a beginning date after this assumption). For the remaining prescriptions, I use the number of refills in each interview period and the beginning and ending months of each interview period

\footnotetext{
${ }^{91}$ Every illness and medical care consumption entry in the data file contains an ICD-9-CM condition code. These codes are used throughout the data cleaning process to match consumption to illness and vice versa.
} 
to randomize a starting date between reasonable bounds.

The last month that a drug is taken is more difficult to determine. The interview period containing each refill of a prescription can be found in the data; however, only the initial fills are accompanied by an exact date. Thus, I know the date that each individual began medication, the number of refills, and the interview periods the refills were purchased in. Using this information and the quantity of each prescription (usually the number of pills and strength of each pill), I approximate the number of months that each refill covers. For the 21 most popular drugs in the data set (see next page) I was able to use average dosage to approximate the number of months. ${ }^{92}$ The approximation provided a length for 2,422 out of the 13,592 total fills/refills purchased over the 2 year period. ${ }^{93}$

For less popular drugs, I use two methods for determining the last month that a drug is taken. First, for drugs in which every refill is the same quantity and consumption spans multiple interview periods, I use the average prescription length in periods prior to the last to calculate an average refill length. I then apply the average length to refills in the last interview period, which provides a length for 4234 additional fills/refills. For single prescription fills and for drugs with non-constant refills (in terms of quantities of pills) I was advised by a medical professional to make the following assumptions:

1. For prescriptions treating acute illnesses, assume prescriptions are for one month.

2. For prescriptions treating chronic illnesses with less than 90 pills, assume prescriptions are for one month.

3. For prescriptions treating chronic illnesses with more than 90 pills or not in a pill form, assume prescriptions are written for three months.

\footnotetext{
${ }^{92}$ Average dosage is taken from "Mosby's: Pharmacology in Nursing." All assumptions were verified by a physician.

${ }^{93}$ This procedure (and others in this section) was done for all fills/refills over the 2 years that an individual was interviewed, even though I only use 1 year of information in estimation. I begin with data covering two years for several reasons. Most importantly, consumption of a prescription in the second year, which began in the first year, tells me that the ending consumption date for that drug runs past the end of the year, providing a solid ending date in the model. Also, because individuals all enter the sample in different months of the year (whichever year their held plan beings) imputation is easier when all prescriptions over the two-year period are analyzed.
} 


\begin{tabular}{|l|l|}
\hline Drug & Average Dosage \\
\hline Birth Control & Monthly packages of 21, 28, and 30 pills \\
\hline Zoloft & $50-150 \mathrm{mg} /$ day \\
\hline Zocor & $5-10 \mathrm{mg} /$ day \\
\hline Zestril & $10-40 \mathrm{mg} /$ day \\
\hline Vasotec & $5-40 \mathrm{mg} /$ day \\
\hline Synthroid & $0.125-0.5 \mathrm{mg} /$ day \\
\hline Prozac & $10-40 \mathrm{mg} /$ day \\
\hline Premarin & $0.3-1.25 \mathrm{mg} /$ day \\
\hline Paxil & $20-50 \mathrm{mg} /$ day \\
\hline Naproxen & $250-500 \mathrm{mg} /$ day \\
\hline Lotensin & $5-40 \mathrm{mg} / \mathrm{day}$ \\
\hline Glucophage & $500-800 \mathrm{mg} / 2-3$ times per day \\
\hline Flonase & 2 sprays/day \\
\hline Claritin & $10 \mathrm{mg} / \mathrm{day}$ \\
\hline Atenolol & $10-100 \mathrm{mg} /$ day \\
\hline Amoxicillin & $500-800 \mathrm{mg} / 2-3$ times per day \\
\hline Toprol & $25-100 \mathrm{mg} /$ day $(\mathrm{max} 400)$ \\
\hline Ranitidine & $150 \mathrm{mg} / 2$ times per day \\
\hline Provera & $5-10 \mathrm{mg} /$ day \\
\hline Norvasc & $5-10 \mathrm{mg} /$ day \\
\hline Ibuprofen & $300-800 \mathrm{mg} / 3-4$ times per day \\
\hline
\end{tabular}

The first assumption is based on the fact that doctors rarely prescribe multi-month prescriptions for acute illnesses. Such illnesses are curable; thus, if the illness continues for more than a month after a prescription has been taken, it is likely that the illness has been misdiagnosed, meaning other treatments need to be explored. The second and third assumptions result from several common prescribing practices. First, many insurance companies set the maximum prescription size that they will cover for particular drugs at 30 or 90 days. As such, 1 month and 3 month prescriptions have become standard prescription lengths for physicians. Second, doctors rarely prescribe less than one pill per day because keeping up with the medication schedule is difficult for patients. Third, longer prescriptions are often prescribed for patients with chronic illnesses because the drug is meant to control the illness, not cure it. Prescriptions are then written for longer periods of time with fewer check-ups needed between refills. 
These assumptions provide a length for every fill/refill in the sample. These lengths are then added together to make a total length for each prescription drug consumption episode. To validate this technique, I review the ending month assigned to each drug to ensure that it falls in the correct interview period (which is observed). The test results in a $90 \%$ success rate. For those predicted to continue their prescription consumption beyond their last known interview period where consumption was reported, interview beginning and ending months and number of refills in the final period are used to randomly impute a more realistic ending month.

\section{B.4 Illness}

In each interview individuals are asked to report all "health problems [experienced during the current interview period] including physical conditions, accidents, or injuries that affect any part of the body as well as mental or emotional health conditions, such as feeling sad, blue, or anxious about something." Participants are told explicitly to include ailments even if they did not seek professional medical care. The individual's description of the illness is recorded as verbatim text, which is later coded to 5-digit ICD-9-CM codes by professional coders. If an illness is identified during the interview as a "priority condition" then an expanded set of questions is asked, including the exact date the illness began. ${ }^{94}$ In all interviews after the first, participants are reminded of illnesses reported in prior interview periods and are asked if the illness has "bothered" them since the beginning of the current interview period. Therefore, I can determine the interview period in which the illness began and ended for all non-priority conditions. For priority conditions, I can determine the exact month than the illness began and the interview period in which it ended.

Before moving on to describe how illnesses are classified and how more precise illness

\footnotetext{
${ }^{94}$ Certain conditions were designated as priority conditions by AHRQ because their prevalence, expense, or relevance to policy called for further inquiry. Some examples are cancer, diabetes, HIV/AIDS, hypertension, arthritis, stomach ulcers, and back problems. A full list can be found on the MEPS website.
} 
dates are determined, the likelihood of unreported illnesses should be considered. In addition to standard recall problems, these data do not contain undiagnosed illnesses. Individuals cannot know about the presence of some illnesses, especially certain chronic illnesses (e.g., hypertension, breast cancer, heart disease, etc.), without the illness being identified by a doctor. The model assumes that illnesses arrive as random (though endogenous) shocks, which do not require a doctor to be identified. Empirically, the missingness of undiagnosed illnesses may be problematic if those rarely seeking medical care, and therefore not being diagnosed, appear healthier than they actually are. ${ }^{95}$ If the objective of this research were to study the role of medical care consumption in determining health over the life course, then this issue of undiagnosed illness would be especially concerning. Data that required participants to take a physical examination, which would reduce the probability of undiagnosed illness, may be more valuable for such an objective. However, the focus of this research is the measurement of moral hazard within a single health insurance year. Endogenous health outcomes are modeled to correct potential bias in moral hazard estimates, but correctly predicting long-term health outcomes is not among the principle objectives. Therefore, for my purposes, the most valuable measure of illness is the measure that an individual is aware of. In the short-run, individuals are most influenced by the illnesses that they actually know they have.

\section{B.4.1 Classification of ICD-9-CM Codes}

To estimate the model, all illnesses must be categorized as acute or chronic. I classify illnesses using the Chronic Condition Indicator (CCI) program, which identifies chronic illnesses by 5-digit ICD-9-CM codes. ${ }^{96}$ For the purposes of this research, a chronic illness differs from an acute illness in that it is assumed to never fully subside. The program defines a chronic illness as "a condition that lasts 12 months or longer and meets one or both of the

\footnotetext{
${ }^{95}$ I thank Jessica Vistnes and Steven Hill of AHRQ for their thoughts and suggestions on this topic.

${ }^{96}$ The Chronic Condition Indicator was developed as part of the Healthcare Cost and Utilization Project (HCUP), which is sponsored by AHRQ.
} 
following tests: (a) it places limitations on self-care, independent living, and social interactions; (b) it results in the need for ongoing intervention with medical products, services, and special equipment." I must then reclassify some of the illnesses observed in the data (see below). ${ }^{97}$

A few of these changes require explanation. ${ }^{98}$ According to the Centers for Disease Control and Prevention (CDC), 16\%, of individuals 14-49 have some form of the Herpes Simplex virus (Genital Herpes), yet most do not know they have it. While it satisfies the definition of chronic illness imposed by this research in that the disease cannot be fully eliminated, it behaves like an acute illness. Infected individuals may experience outbreaks a few times a year, but potentially not at all. During these episodes individuals use medical care to treat the symptoms, but outside these outbreaks live normal lives. Furthermore, the rare occurrence of this illness in the data suggest that the individuals only report the virus during an outbreak, meaning it is also reported as an acute illness. Carpel Tunnel syndrome is coded as an acute illness because treatment (splint, physical therapy, or surgery) can restore the wrist to full health. The other respiratory disease recode applied to only one individual, who reports having had this disease for 15 years. ${ }^{99}$

\footnotetext{
${ }^{97}$ This list is not an exhaustive list of all ICD-9-CM codes that should be reclassified given the difference in chronic illness definitions. The illnesses listed here are only the observed illnesses that need to be changed.

${ }^{98}$ None of these assumptions are likely to have a large impact in estimation. Each of these changes affects fewer than 25 individuals, except Acute reaction to stress and Chronic Sinusitis, which still affect fewer than 75 individuals.

${ }^{99}$ Other notes: For the purposes of this research, near/far sightedness and astigmatism are not considered illnesses though they appear frequently in the condition files. Only 198 of the 6208 reported medical conditions are missing an ICD-9-CM code. For these individuals, I look at medical care consumption in the same periods to try to infer what the illness was. If there is no medical care consumption in the period and the illness is present in every period over the two year span, I assume that it is a chronic illness. Otherwise, I assume that it is an acute illness.
} 


\begin{tabular}{|l|l|l|}
\hline ICD-9-CM & Illness & Changed to \\
\hline 054 & Herpes Simplex & Acute \\
\hline 239 & Unspecified cancer & Chronic \\
\hline 308 & Acute reaction to stress & Acute \\
\hline 309 & Adjustment reaction & Acute \\
\hline 354 & Carpel Tunnel & Acute \\
\hline 360 & Disorder of the globe & Acute \\
\hline 436 & Acute Cerebrovascular Disease & Acute \\
\hline 473 & Chronic Sinusitis & Acute \\
\hline 474 & $\begin{array}{l}\text { Chronic disease of the tonsils and } \\
\text { adenoids }\end{array}$ & Acute \\
\hline 519 & Other respiratory disease & Chronic \\
\hline 562 & Diverticula of the intestines & Acute \\
\hline 625 & Premenstrual Syndrome & Acute \\
\hline 730 & Unspecified Osteomyelitis & Acute \\
\hline V10 & Past cancer & Chronic \\
\hline
\end{tabular}

\section{B.4.2 Illness Beginning and Ending Dates}

The model requires that the data reveal whether or not an individual has an acute illness and chronic illness in each of the 12 months of the insurance year. To determine this information, I need to know the beginning and ending month of each reported illness. For every illness reported in the data, it can be determined in which interview period the illness began and ended, but not necessarily the month. Thus, in what follows, I describe the procedures used to impute the beginning and ending month for each illness. I begin with a few simplifying assumptions. First, all congenital diseases observed in the data are assumed to begin prior to the beginning of the insurance year. ${ }^{100}$ Second, I assume that all illnesses reported in consecutive interview periods represent one continuous illness. Furthermore, throughout the file there are examples of the same illness being reported multiple times by the same individual with gaps in reporting (e.g., had illness during 1st and 3rd interview). At times, it is reasonable to assume that the multiple records describe one continuous illness. Most of the time, it is unlikely that the illness lasted 6 or 7 months, so it is best assumed that the illness

\footnotetext{
${ }^{100}$ I observe the following ICD-9-CM codes: 747, 753, 755, 757, 758, 759.
} 
occurred on two separate occasions (e.g., a common cold). I resolve these issues case by case. In total, there are 414 instances where a gap rule is needed (two entries for the same illness and an interview gap between reports). Rules are generally made at the illness level (e.g., any gap for a common cold implies two illnesses, for which there are 100). After these edits, I observe 5586 independent illnesses for the estimation sample over the 2 year period of study. I add to these illnesses 37 illness records that are necessary to match medical care consumption information (e.g., I observe that an individual takes insulin in every month but has no record of diabetes). I then remove redundant illnesses, or multiple records of illnesses that last the exact same amount of time and likely describe the same ailment (e.g., if I observe an individual with diarrhea and a stomach ache in the same interview period, I drop one of these records). ${ }^{101}$ I also remove any illness known to begin after the end of the insurance year. This step reduces the number of illnesses to 4,482 .

Of these illnesses, 29\% are classified by AHRQ as "priority conditions" (mostly chronic and important acute illnesses), so the month that the illness began is known. A beginning date must be imputed for the remaining illnesses. To aid in imputation, I first assume that if the individual consumed medical care for an illness in the first interview period that an illness is reported, then the month of consumption is the month that the illness began. This assumption is equivalent to assuming that people do not wait more than a month to go to the doctor if they are going to go at all. After this assumption, 1,299 illnesses are in need of a beginning month. For these, a beginning date is drawn uniformly between the first and last month of the interview where the illness was first reported. For the first interview period, I account for the fact that the illness may have begun prior to the start of the year. This randomization procedure results in 302 illnesses having start dates that are past the end of the insurance year.

\footnotetext{
${ }^{101}$ Most of instances of redundant illnesses result from multiple symptoms of an illness being reported as an actual illness. Removing redundant illnesses relieves some of the burden of imputation. The model does not distinguish between 1 and 2 acute illnesses in a month, so even if I were to keep both illness records the information would eventually be condensed. However, dropping a record means I do not have to determine a starting and ending date for the illness.
} 
They are dropped and 4,180 illnesses remain.

Illness ending months are more difficult to extract because they are never observed explicitly. I only observe the last interview period in which an illness was reported. Fortunately, chronic illnesses are assumed to never end, so only acute illnesses need an approximated ending month. Further, many acute illnesses begin during the 12 months of the insurance year but end in an interview period after the close of the insurance year, meaning no ending month is required for the model. Thus, only 2,592 illnesses need an ending date. Randomization is not necessary for 343 illnesses because they start and end in the same interview period and the beginning date is the last month of the period. There are then only 2,249 illnesses that need an ending month to be imputed. For these, I draw an ending month at random from the interview period that the illness reportedly ended in. If an illness ends in the same interview period that it begans, the lower bound is set to the beginning month. ${ }^{102}$ The distribution of bound lengths is shown below.

\begin{tabular}{|c|c|c|}
\hline $\begin{array}{c}\text { Bound } \\
\text { Length }\end{array}$ & Frequency & $\begin{array}{c}\text { Cumulative } \\
\text { Percentage }\end{array}$ \\
\hline 1 & 355 & $15.8 \%$ \\
\hline 2 & 437 & $35.2 \%$ \\
\hline 3 & 391 & $52.6 \%$ \\
\hline 4 & 368 & $69.0 \%$ \\
\hline 5 & 371 & $85.5 \%$ \\
\hline 6 & 197 & $94.2 \%$ \\
\hline 7 & 101 & $98.7 \%$ \\
\hline 8 & 29 & $100.0 \%$ \\
\hline Total & 2249 & \\
\hline
\end{tabular}

\footnotetext{
${ }^{102}$ The lower bound could be increased (decreasing the draw range) by setting it equal to the month of last corresponding consumption in the last interview period that the illness was reported. I did not use this strategy because it almost certainly would have lead estimates to reflect medical care as harmful for ones health, as those consuming medical care would on average have longer illness periods than others. With the method used, the productivity of medical care is identified by the data alone. If medical care helps an individual avoid illness in the following interview period, then it is productive. If it does not, then medical care may be harmful.
} 


\section{APPENDIX: OUT-OF-POCKET EXPENDITURE EQUATION}

Out-of-pocket expenditure resulting from $v_{t}$ trips to the doctor, $s_{t}$ hospital days, and consuming prescription drugs $r_{t}$ in month $t$ can be calculated as a function of accumulated outof-pocket doctor's office expenditure entering the month, $A D E_{t}$; accumulated out-of-pocket hospital expenditure entering the month, $A H E_{t}$; the month $t$ total price of a doctor's office visit, hospital days, and prescription drug consumption, $p_{t}^{v}, p_{t}^{s}$, and $p_{t}^{r}$ respectively; and the cost-sharing features of one's insurance plan. The cost-sharing features that may impact the out-of-pocket cost of care are

- $D_{1}^{d}=$ doctor's office specific deductible

- $D_{1}^{h}=$ hospital specific deductible

- $D_{2}=$ stop loss

- $C_{1}^{d}=$ doctor's office co-insurance rate

- $C_{2}^{d}=$ doctor's office co-pay level

- $C_{1}^{h}=$ hospital co-insurance rate

- $C_{2}^{h 1}=$ hospital co-pay level defined by day

- $C_{2}^{h 2}=$ hospital co-pay level defined by stay

- $C_{3}=$ prescription drug co-insurance level

To ensure that the out-of-pocket cost of an additional unit of care is captured regardless of the unique combination of insurance features a plan contains, I categorize each plan into one of four general types and allow different pieces of the function below to change depending on the plan type. These four types are

- Type 1: composite deductible (or no deductible at all) and hospital co-pay defined by day (or hospital co-pay does not at all exist). 
- Type 2: composite deductible (or no deductible at all) and hospital co-pay defined by stay.

- Type 3: separate deductible(s) and hospital co-pay defined by day (or hospital co-pay does not at all exist).

- Type 4: separate deductible(s) and hospital co-pay defined by stay.

The strategy for calculating out-of-pocket expenditure is to separately determine the amount of pre-deductible and post-deductible expenditure first for doctor visits and then for hospital days. Doctor and hospital expenditure must be derived separately because the cost-sharing features of nearly every plan require individuals to pay a different proportion of the total cost for each service. Two assumptions are needed to calculate out-of-pocket expenditure each month. First, it is assumed that the total price charged for a doctor visit and the total price charged for a hospital day are fixed for an individual within any month. This assumption makes the problem empirically tractable. As mentioned, an individual solves his optimization problem by integrating over three distributions of possible prices. If this price were allowed to vary for each trip to the doctor/hospital in a month, then a $V * S$ dimensional integral would need to be solved in every month. Further, given that the factors influencing the total prices one faces in a month are not changing, it seems reasonable to assume that the total price does not change. Second, the equation assumes that all doctor visits within a month are made before any hospital decisions take place. While this assumption is clearly not ideal, it is necessary if one hopes to avoid modeling the specific order in which an individual decides to visit the doctor and hospital, which would cause the size of the alternative set to explode. ${ }^{103}$

Conditional on these assumptions, out-of-pocket expenditure can be calculated as follows:

\footnotetext{
${ }^{103}$ Order is important because it determines when exactly during the month the deductible or stop loss is passed. Alternatives to this assumption are: (1) Force an individual to select not only the number of visits but the order as well; however, this is likely to be empirically infeasible. (2) Stipulate that non-linear changes in medical care prices that happen when one crosses the deductible or stop loss only occur between months, which would greatly simplify the budget constraint; however, it would remove important variation from the data.
} 
$\underline{\text { Pre-deductible spending on doctor's office care is: }}$

$$
o_{t}^{1 d}\left(v_{t}, p_{t}^{v}, \Psi_{t}\right)= \begin{cases}p_{t}^{v} * v_{t} & \text { if } 0 \leq p_{t}^{v} * v_{t} \leq x_{t}^{1} \\ x_{t}^{1} & \text { if } 0 \leq x_{t}^{1}<p_{t}^{v} * v_{t}\end{cases}
$$

where

Type 1: $x_{t}^{1}=$ composite deductible remaining $=\max \left\{0, D_{1}-A D E_{t}-A H E_{t}\right\}$

Type 2: $x_{t}^{1}=$ composite deductible remaining $=\max \left\{0, D_{1}-A D E_{t}-A H E_{t}\right\}$

Type 3: $x_{t}^{1}=$ doctor deductible remaining $=\max \left\{0, D_{1}^{d}-A D E_{t}\right\}$

Type 4: $x_{t}^{1}=$ doctor deductible remaining $=\max \left\{0, D_{1}^{d}-A D E_{t}\right\}$

Post-deductible spending on doctor visits is:

$$
o_{t}^{2 d}\left(v_{t}, p_{t}^{v}, \Psi_{t}\right)= \begin{cases}\operatorname{over}_{t}^{d} & \text { if } \text { over }_{t}^{d} \leq z_{t}^{1} \\ \text { over }_{t}^{d}-z_{t}^{1} & \text { if } \text { over }_{t}^{d}>z_{t}^{1}\end{cases}
$$

where

$$
\begin{aligned}
& \text { over }_{t}^{d}=\max \left\{0, \operatorname{int}\left\{\frac{\left(p_{t}^{v} * v_{t}\right)-x_{t}^{1}}{p_{t}^{v}}\right\} * C_{2}^{d}\right\}+ \\
& \quad \max \left\{0,\left(p_{t}^{v} * v_{t}\right)-x_{t}^{1}-\operatorname{int}\left\{\frac{\left(p_{t}^{v} * v_{t}\right)-x_{t}^{1}}{p_{t}^{v}}\right\} C_{2}^{d}\right\} * C_{1}^{d} \\
& z_{t}^{1}=\text { stop loss remaining }=\max \left\{0, D_{2}-A D E_{t}-A H E_{t}-o_{t}^{1 d}\right\}
\end{aligned}
$$

Total amount spent out-of-pocket on doctor visits in month $t$ is:

$$
O_{t}^{d}=o_{t}^{1 d}+o_{t}^{2 d}
$$


Conditional on month $t$ doctor spending, pre-deductible spending on hospital care is:

$$
o_{t}^{1 h}\left(s_{t}, p_{t}^{s}, \Psi_{t}\right)= \begin{cases}p_{t}^{s} * s_{t} & \text { if } 0 \leq p_{t}^{s} * s_{t} \leq x_{t}^{2} \\ x_{t}^{2} & \text { if } 0 \leq x_{t}^{2}<p_{t}^{s} * s_{t}\end{cases}
$$

where

Type 1: $x_{t}^{2}=$ composite deductible remaining $=\max \left\{0, D_{1}-A D E_{t}-A H E_{t}-O_{t}^{d}\right\}$

Type 2: $x_{t}^{2}=$ composite deductible remaining $=\max \left\{0, D_{1}-A D E_{t}-A H E_{t}-O_{t}^{d}\right\}$

Type 3: $x_{t}^{2}=$ hospital deductible remaining $=\max \left\{0, D_{1}^{h}-A H E_{t}\right\}$

Type 4: $x_{t}^{2}=$ hospital deductible remaining $=\max \left\{0, D_{1}^{h}-A H E_{t}\right\}$

Post-deductible spending on hospital care is:

$$
o_{t}^{2 h}\left(s_{t}, p_{t}^{s}, \Psi_{t}\right)= \begin{cases}\text { over }_{t}^{h} & \text { if } \text { over }_{t}^{h} \leq z_{t}^{2} \\ \text { over }_{t}^{h}-z_{t}^{2} & \text { if } \text { over }_{t}^{h}>z_{t}^{2}\end{cases}
$$

where

$$
z_{t}^{2}=\text { stop loss remaining }=\max \left\{0, D_{2}-A D E_{t}-A H E_{t}-O_{t}^{d}-o_{t}^{1 h}\right\}
$$

$A=\operatorname{int}\left(\frac{\left(p_{t}^{s} * s_{t}\right)-x_{t}^{2}}{p_{t}^{s}}\right)$, where $\operatorname{int}(w)$ rounds $w$ to the nearest integer

Type 1: over $_{t}^{h}=\max \left\{0, A * C_{2}^{h 1}\right\}+$

$$
\max \left\{0,\left(p_{t}^{s} * s_{t}\right)-x_{t}^{2}-A * C_{2}^{h 1}\right\} * C_{1}^{h}
$$

Type 2: over $_{t}^{h}=\max \left\{0, \min \left\{\left(p_{t}^{s} * s_{t}\right)-x_{t}^{2}, C_{2}^{h 2}\right\}\right\}+$

$$
\max \left\{0,\left(p_{t}^{s} * s_{t}\right)-x_{t}^{2}-C_{2}^{h 2}\right\} * C_{1}^{h}
$$

Type 3: over $_{t}^{h}=\max \left\{0, A * C_{2}^{h 1}\right\}+$

$$
\max \left\{0,\left(p_{t}^{s} * s_{t}\right)-x_{t}^{2}-A * C_{2}^{h 1}\right\} * C_{1}^{h}
$$


Type 4: over $_{t}^{h}=\max \left\{0, \min \left\{\left(p_{t}^{s} * s_{t}\right)-x_{t}^{2}, C_{2}^{h 2}\right\}\right\}+$

$$
\max \left\{0,\left(p_{t}^{s} * s_{t}\right)-x_{t}^{2}-C_{2}^{h 2}\right\} * C_{1}^{h}
$$

The total amount spent out-of-pocket on hospital care in month $t$ is:

$$
O_{t}^{h}=o_{t}^{1} h+o_{t}^{2} h
$$

Out-of-pocket expenditure in month $t$ is:

$$
O_{t}=O_{t}^{d}+O_{t}^{h}+\left(r_{t} * p_{t}^{r} * C_{3}\right)
$$

Accumulated doctor and hospital expenditure entering month $t+1$ is:

$$
\begin{aligned}
& A D E_{t+1}= \begin{cases}A D E_{t}+O_{t}^{d} & \text { if } t>0 \\
0 & \text { if } t=0\end{cases} \\
& A H E_{t+1}= \begin{cases}A H E_{t}+O_{t}^{h} & \text { if } t>0 \\
0 & \text { if } t=0\end{cases}
\end{aligned}
$$




\section{APPENDIX: WELFARE EXPERIMENTS}

Until now, this manuscript has focused on how medical care expenditure responds to various sequences of medical care prices, which are caused by different health insurance cost-sharing structures. While this relationship between insurance and expenditure is interesting for policy-makers, little has been said about the impact that insurance has on welfare, which is of greater interest to economists. In this section, I discuss the (consumer) welfare implications of insurance possession for individuals in my estimation sample under the insurance environment observed in the data. I also explore several ways in which the insurance environment may be altered in order to improve consumer welfare.

\section{D.1 The Welfare Implications of Insurance Possession}

The purpose of health insurance is to protect risk averse individuals from the financial risk associated with negative health shocks. If medical care consumption were unaffected by insurance possession, this risk protection would yield a welfare gain. However, because insurance decreases the financial disincentive of medical care consumption (by lowering the price), insurance encourages medical care consumption to increase (known as moral hazard). Therefore, if the level of medical care consumption without insurance (when individuals pay the full price of medical care) is socially optimal, then the additional consumption induced by insurance is welfare reducing. Furthermore, the welfare gains from risk protection and the welfare losses from overconsumption are inversely related. Any plan providing more financial protection, by lowering an individual's out-of-pocket price of care, at the same time encourages more over-consumption. The socially optimal level of insurance coverage must then balance the trade-off between welfare gains from risk protection and welfare losses from moral hazard (Arrow 1963; Pauly 1968).

Several researchers have estimated the relative size of these effects, all conditioning on the important assumption that the level of medical care consumption without insurance is socially 
optimal (Feldstein 1973; Feldman and Dowd 1991; Feldstein and Gruber 1995; Manning and Marquis 1996; Finkelstein and McKnight 2008; Kowalski 2013). The assumption is strict, as it requires that there are no externalities from the additional medical care consumption induced by insurance (e.g., the reduction of communicable diseases) and that medical care prices in a market without insurance are competitive. While I find each of these requirements troubling, a richer economic model than the one presented in this research is needed in order to relax them. Therefore, I discuss below how to calculate the welfare gains from risk protection and the welfare losses from moral hazard assuming that consumption when uninsured is socially optimal. While more work must be done to relax this critical assumption, this is the first unified model of within-year medical care behavior and health transitions that is able to estimate these effects of insurance possession. Given my hesitance to accept the underlying assumptions, I explain the procedure but do not actually calculate the welfare effects.

\section{D.1.1 Welfare Gains from Risk Protection}

Calculating the welfare gains from risk protection using this model is easier than calculating the welfare losses from moral hazard, so I discuss it first. Insurance serves to protect individuals from financial risk, not the risk of a poor health shock. In the model, individuals make medical care consumption decisions by integrating over three price distributions. Insurance improves welfare by limiting the individual's exposure to these price distributions; intuitively, insurance truncates the right tails of these distributions. The welfare gains from risk protection can then be calculated as the willing to pay for insurance, where the benefit of insurance is limited to the relative gain in within period expected utility due to the truncated price distributions. ${ }^{104}$

Therefore, in order to use this model to calculate the welfare gains from risk protection for an individual $i$, one would first simulate the model for all individuals under no insurance.

\footnotetext{
${ }^{104}$ The relevant welfare gain is in expected utility, and not realized utility, because the gains from risk protection are the product of the truncated price distributions, not lower realized prices.
} 
Then, one would simulate the model for all individuals under their observed insurance plan, while assuming a premium of zero and that the medical care consumption path for each individual mimics the path under the no insurance condition. This assumption is necessary if the utility differences due to insurance are to be caused by changes in risk protection only. Otherwise, consumption would increase with insurance, as it decreases the price of care. One can then compare utility with and without insurance, where utility is calculated as the sum of expected utilities in each month. (Expected utility is calculated at observed choices by integrating over the price distributions.) Finally, the welfare gain from risk protection is calculated as the willingness-to-pay for insurance protection, which can be thought of as the premium that would cause utility when insured to be equal to utility when uninsured.

\section{D.1.2 Welfare Losses from Moral Hazard}

When individuals become insured, the price of medical care falls and consumption of medical care increases. In the empirical health economics literature, the change in annual expenditure resulting from this increase in consumption is often referred to as moral hazard. Assuming consumption without insurance (i.e., when individuals pay the full price of care) is socially optimal, this additional consumption generates a welfare loss. However, the entire increase in expenditure is not representative of a welfare loss. As is covered extensively by Nyman (1999a,b,c), the increase in consumption is the result of both lower prices and an

income transfer from the well to the sick, the latter of which is welfare neutral. The point is made clear in the following example:

"Assume a large population of 10,000 consumers, each with identical preferences and incomes of US $\$ 40,000$. The probability that each consumer will become ill is $1 / 10$.... With insurance that pays off by reducing the price from US\$1 per unit of medical care to US $\$ 0$ per unit, each ill consumer who is insured consumes US $\$ 20,000$ worth of medical care $(20,000$ units) after paying a US $\$ 2000$ premium. ... Of this US $\$ 20,000$, US $\$ 2000$ represents the ill consumer's contribution to the insurance pool and US\$18,000 represents income transfers from those who remain healthy. ... As a result, the ill consumer now is consuming a total of US $\$ 58,000$ worth of both medical care and other consumption, but had 
only US $\$ 40,000$ to spend without insurance. The only way that this person can spend US $\$ 58,000$ with an original budget of US $\$ 40,000$ is because of a transfer of US\$18,000 in income from those nine (out of every 10 consumers) who remain healthy. ... The increased spending on health care that occurs as a result of this income transfer should be excluded from the welfare calculations, as is typically done with income transfers, because the gain to one individual (US\$18,000 in this case) equals the loss to others (US\$2000 by each of the nine other consumers). Only that portion of medical care spending that remains and that is due to a pure price effect has welfare implications." Nyman (1999a)

Of the papers mentioned in Section D.1, all but Kowalski (2013) calculate the welfare losses from moral hazard including the income transfer. Using the model presented in this dissertation, one can calculate these welfare losses both with (for comparison purposes) and without medical care consumption due to income transfers. The simulation is best understood with reference to Figure D.1. In the figure, a representative individual uses his income, $Y$, to consume medical care, $M$, and a composite good, $X$. Without insurance, each good is priced such that the slope of the budget constraint $B C_{1}$ reflects the relative price per unit. His preferences are described by indifference curve $I C_{1}$, so that when ill and uninsured he consumes $A$ units of medical care (when well he does not consume any medical care). He is offered an insurance plan defined by premium $P$, deductible $D_{1}$, co-insurance rate $a \in$ $(0,1)$, and stop loss $D_{2} \cdot{ }^{105}$ When insured and ill, the individual's preferences dictate that he consumes $B$ units of medical care. According do the above definition, moral hazard is then calculated as the difference between $\mathrm{A}$ and $\mathrm{B}$, where part of the spending increase is due to the price decrease (substitution effect) and part is due to income transfers (income effect). To calculate the welfare loss from moral hazard, these two effects must be separated.

To determine the increase is consumption due to the price change only, the income advantage of the insured must be removed. One can do so by shifting the insured budget constraint

\footnotetext{
${ }^{105}$ The cost-sharing structure of this health insurance plan is standard in todays market. Individuals cover the full cost of care up to some level called the deductible $\left(D_{1}\right)$. After crossing the deductible, they are responsible for only a portion of the cost of care. For simplicity, I assume this portion is determined by a co-insurance rate ( $a$, a percentage of the cost), rather than a co-pay level (a fixed number of dollars). If accumulated out-ofpocket expenditure over the insurance year eventually reaches the stop loss $\left(D_{2}\right)$, then all medical care has zero out-of-pocket cost for the remainder of the year.
} 
Figure D.1: Income and Substitution Effects

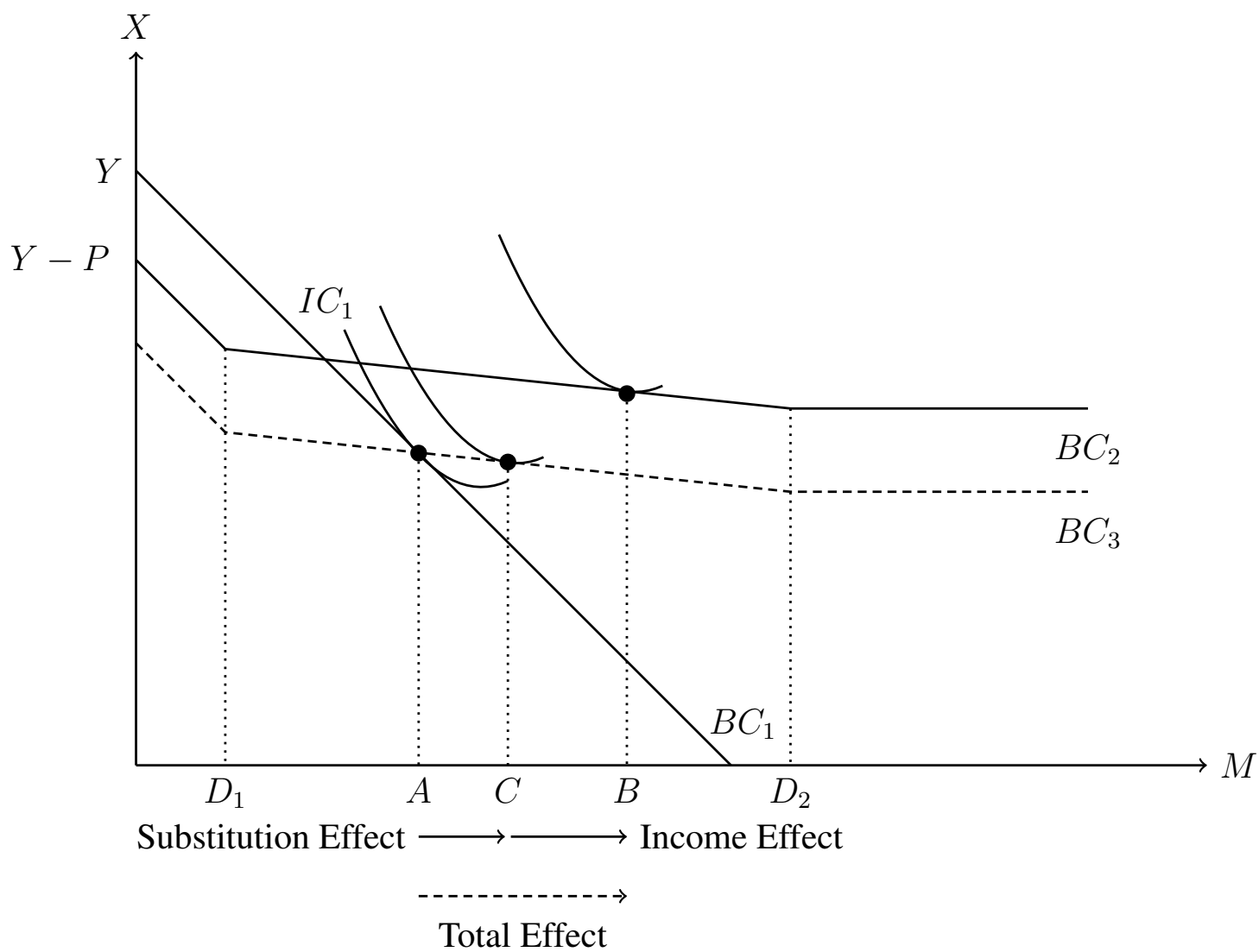

down to $B C_{3}$ so that it is binding at medical care consumption level $A$, optimal consumption when uninsured. ${ }^{106}$ Optimal medical care consumption at $B C_{3}$, the income neutral budget constraint, is $C$. Thus, $C$ minus $A$ measures the increase in medical care consumption due to a fall in prices, while $B$ minus $C$ measures the increase due to income transfers. The welfare loss from this additional consumption $(C$ minus $A$ ) can then be calculated as the total expenditure on medical care at consumption level $C$ minus an individuals willingness to pay for the consumption level. (At $A$, total expenditure equals willingness to pay.)

In order to calculate this difference for an individual $i$, one would first determine medical care consumption level $A$ for each individual by simulating the model under no insurance.

\footnotetext{
${ }^{106}$ The vertical shift from $B C_{2}$ to $B C_{3}$ reflects the additional amount of composite consumption enjoyed by an uninsured individual, consuming at $A$, after he becomes insured. This additional composite consumption reflects added wealth from insurance.
} 
Then, the increase in composite consumption with insurance, $C C$, assuming no moral hazard (graphically, this is the vertical distance between $B C_{2}$ and $B C_{3}$ ) can be calculated by simulating the model for all individuals under their observed insurance plan, while holding medical care consumption fixed. After calculating $C C$ one can simulate the model for all individuals under their observed insurance plan assuming (1) the plan premium is $P+C C$, so that their budget constraint in this step reflects that of $B C_{3}$, and (2) individuals choose medical care optimally. This simulation generates medical care consumption level $C$ for each individual, as well as the corresponding total expenditure calculation needed for the welfare calculation. Finally, one can calculate individual willingness to pay for the consumption stream $C$ and compare it to total expenditure.

\section{D.2 The Welfare Implications of Limited Insurance Choice}

The United States is unique among developed countries in its lack of public health insurance coverage for non-elderly citizens. ${ }^{107}$ Most (62\%) non-elderly Americans carry private health insurance coverage. Also, U.S. tax law encourages employers to select and subsidize a set of plans for their employees to choose from. ${ }^{108}$ The result of this law is that $56 \%$ of the non-elderly population carries a health insurance plan from an alternative set selected and subsidized by their employer. ${ }^{109}$

Unfortunately, many employers offer a very limited set of health insurance alternatives, if they offer a choice at all. According to the 2013 Employer Health Benefits Survey, 87\% of the U.S. employers who offer health insurance offer only one type of plan and $12 \%$ offer

\footnotetext{
${ }^{107}$ According to OECD Health Data, only $26.4 \%$ of the U.S. population in 2009 held public health insurance coverage; the lowest among OECD countries. Chile had the second lowest level of public coverage at $73.5 \%$. Of the 30 member nations, 25 extend public health insurance coverage to over $95 \%$ of their citizens.

${ }^{108}$ U.S. tax law allows employers to offer a set of insurance plans in which they pay all or a portion of their employee's insurance premiums with dollars that are not subject to the federal income tax. As a result, employees prefer to acquire health insurance through their employer, rather than on the private market where they would have to pay premiums with after (federal income) tax dollars.

${ }^{109}$ The remaining non-elderly population is either uninsured (18\%), has Medicaid coverage (18\%), or gets coverage through some other public source (3\%, Current Population Survey 2013).
} 
two alternatives. ${ }^{110}$ In the estimation sample, $42 \%$ of individuals are limited to one plan, $18 \%$ have access to two plans, and $13 \%$ select from three plans. And while there is much debate in popular media on the current health insurance environment in the U.S., little attention has been given to the welfare implications of these limited alternative sets. Furthermore, Dafny, Ho, and Varela (2013) are the only scholars to study these welfare effects in the health economics literature.

I am able to examine the welfare implications of the limited health insurance alternative sets observed in the estimation sample by calculating individuals' willingness to pay for counterfactual health insurance plans. I focus the analysis on adding two particular plans, in separate simulations, in order to determine if the limited alternative set generally results in over or under insurance. First, I add a high deductible (HD) health insurance plan to every individuals' alternative set. The HD plan has a \$1000 deductible and no cost-sharing after the deductible is met, which is consistent with the HD plans observed in the data. Second, I add a comprehensive plan (i.e., individuals pay nothing out-of-pocket for care) to every individuals' alternative set. For each of these plans, the total premium charged and the proportion of that premium that individuals must pay out-of-pocket determines the rate at which the plans are selected. Therefore, I vary insurance premiums and employer contributions across simulations. The HD plan provides little coverage for insured individuals, so the total premiums tested are taken from the left tail of the total premium distribution in the data. (I use the minimum observed premium and premiums at the $1 \%, 5 \%, 10 \%$, and $20 \%$ percentiles.) Conversely, the comprehensive plan provides full coverage for insured individuals, so the total premiums tested are taken from the right tail of the total premium distribution in the data. (I use premiums at the $80 \%, 90 \%, 95 \%$, and $99 \%$ percentiles and the maximum premium observed in the data.) For each plan-premium pair, I simulate behavior assuming both that

\footnotetext{
${ }^{110}$ Equal proportions of employees are not limited to these alternative sets as small firms are more likely to offer small alternative sets. Because more individuals work for large firms than small firms, $49 \%$ of all covered employees are limited to one type of plan, $36 \%$ to two types of plans, and $16 \%$ to three of more types of plans.
} 
individuals receive no premium assistance from their employer and that their employer contributes the average amount they are observed to contribute in the data (i.e., if employer A contributes $\$ 500$ a year to plan 1 and $\$ 700$ a year to plan 2, then they contribute $\$ 600$ a year to the new plan).

The results of these simulations can be seen in Table D.1. For each simulation, I report the percentage of the population that remains uninsured, the percentage of the population that selects the new plan, and the average (consumer) welfare gain in the population due to the new plans inclusion. Prior to adding the new plan, 9.7\% of the population chose to be uninsured. Added consumer welfare for individuals who do not select the new plan is zero. For individuals who select the new plan, welfare is calculated as the individual's willingness to pay for the new plan minus their out-of-pocket payment. The welfare gain is then averaged across the population. 
Table D.1: Individual Response to Additional Plans

\begin{tabular}{|c|c|c|c|c|c|c|}
\hline \multirow[b]{2}{*}{$\begin{array}{c}\text { Total } \\
\text { Premium }\end{array}$} & \multicolumn{3}{|c|}{ No Employer Contribution } & \multicolumn{3}{|c|}{ Average Employer Contribution } \\
\hline & $\begin{array}{l}\text { Precent } \\
\text { Uninsured }\end{array}$ & $\begin{array}{l}\text { New Plan } \\
\text { Take-up }\end{array}$ & $\begin{array}{c}\text { Average } \\
\text { Welfare Gain }\end{array}$ & $\begin{array}{l}\text { Precent } \\
\text { Uninsured }\end{array}$ & $\begin{array}{c}\text { New Plan } \\
\text { Take-up }\end{array}$ & $\begin{array}{c}\text { Average } \\
\text { Welfare Gain }\end{array}$ \\
\hline & \multicolumn{6}{|c|}{ High Deductible Plan Added } \\
\hline$\$ 29(\min )$ & $6.1 \%$ & $20.3 \%$ & $\$ 221$ & $6.0 \%$ & $21.0 \%$ & $\$ 228$ \\
\hline$\$ 800(1 \%)$ & $8.4 \%$ & $9.2 \%$ & $\$ 115$ & $6.2 \%$ & $20.4 \%$ & $\$ 222$ \\
\hline$\$ 1395(5 \%)$ & $8.8 \%$ & $5.3 \%$ & $\$ 73$ & $6.7 \%$ & $18.4 \%$ & $\$ 204$ \\
\hline$\$ 1536(10 \%)$ & $9.1 \%$ & $4.8 \%$ & $\$ 66$ & $6.8 \%$ & $17.6 \%$ & $\$ 197$ \\
\hline \multirow[t]{2}{*}{$\$ 1716(20 \%)$} & $9.2 \%$ & $4.1 \%$ & $\$ 58$ & $7.3 \%$ & $16.2 \%$ & $\$ 185$ \\
\hline & \multicolumn{6}{|c|}{ Comprehensive (No Cost-Sharing) Plan Added } \\
\hline$\$ 2496(80 \%)$ & $8.7 \%$ & $9.6 \%$ & $\$ 177$ & $6.0 \%$ & $32.6 \%$ & $\$ 503$ \\
\hline$\$ 2988(90 \%)$ & $9.0 \%$ & $7.0 \%$ & $\$ 137$ & $7.1 \%$ & $23.9 \%$ & $\$ 381$ \\
\hline$\$ 3456(95 \%)$ & $9.2 \%$ & $5.2 \%$ & $\$ 107$ & $7.9 \%$ & $17.6 \%$ & $\$ 294$ \\
\hline$\$ 4587(99 \%)$ & $9.4 \%$ & $2.7 \%$ & $\$ 64$ & $8.8 \%$ & $8.8 \%$ & $\$ 162$ \\
\hline$\$ 10188(\max )$ & $9.6 \%$ & $0.0 \%$ & $\$ 11$ & $9.6 \%$ & $0.0 \%$ & $\$ 17$ \\
\hline
\end{tabular}

$\dagger$ The table contains four quadrants. The top left quadrant represents measured responses to the addition of a high deductible (HD) plan to all individuals' insurance alternative sets when employers pay no part of the premium; in the top right employers make the average contributions they are observed to make in the data. The bottom two quadrants measure responses to the addition of a comprehensive plan rather than an HD plan. Interpretation of the table elements can be understood by considering the first three entires $(6.1 \%, 20.3 \%$, $\$ 221$ ). If an HD plan is added to each individuals' alternative set, the plan carries a total premium of $\$ 29$, and employers pay none of this premium, then (1) the percentage of the population that is uninsured falls from $9.7 \%$ to $6.1 \%$; (2) $20.3 \%$ of the total population selects the HD plan over their other alternatives; (3) the average welfare gain in the population is $\$ 221$ per person.

$\ddagger$ The information in parenthesis in the far left column represents the percentile of the observed total premium distribution from which the corresponding premium was taken. For example: $\$ 3456$ is the 95th percentile of the observed total premium distribution. Also, for both insurance selection and welfare purposes, the minimum out-of-pocket premium that an individual faces is zero. For example: If the total annual HD premium is $\$ 29$ and his employer contributes $\$ 1000$ on average to insurance premiums, then the out-of-pocket premium used in analysis (when the employer contributes) is $\$ 0$. 
There are several patterns in Table D.1. First, when employers contribute to premiums plans are effectively cheaper for individuals, so the uninsured rate falls, take-up of the new plan increases, and the average welfare gain increases. Second, the total premium charged for the new plans is an important determinate of individual responses. For each plan and employer contribution arrangement, as the total premium increases the percentage of uninsured individuals increases, take-up decreases, and average welfare gain falls. Note also that exploring the inadequacy of the observed alternative sets also requires one to know more about what constitutes a reasonable price for the new plans. Certainly if full coverage is offered at a low premium, then many individuals will choose it, but this does not provide convincing evidence that individuals are underinsured at present. Only when an appropriate price is charged for the new plans can this be determined.

Consider the following: Let us assume that the new plans represent potential public insurance options that would be offered by the federal government with the same tax privileges as employer provided plans. For each of these plans, there is some total premium that the government would charge to generate zero profits. Assume that the government would break even with a premium at the 10th percentile of observed premiums (\$1536) for the HD plan and at the 90th percentile (\$2988) for comprehensive coverage. If employers contributed to employee premiums at their current level, then $17.6 \%$ of the population would take-up the HD plan if it alone were offered and $23.9 \%$ would take up comprehensive coverage if it were offered instead. Furthermore, the average welfare gain generated by offering comprehensive coverage outweighs that generated by offering the HD plan. Both of these findings would suggest that the more significant effect of limited insurance alternative sets is underinsurance, rather than overinsurance. However, it is also the case that the proportion of uninsured individuals is reduced more by offering the HD plan than the comprehensive plan. So while the insured population may desire more comprehensive coverage, the uninsured population is in greater need of affordable, low generosity coverage.

In order to use this model for reliable policy analysis; such as determining the optimal 
public option to offer, more attention must be paid to equilibrium effects in the supply of both insurance and medical care. The above conclusions are only as reliable as the assumptions made on what premiums would be charged for the new plans. If comprehensive coverage requires a premium at the 99 th percentile, rather than the 90 th, then the result changes entirely. Also, this analysis ignores the equilibrium effects that shifts in insurance demand have on premiums. For example, the inclusion of an HD plan to all alternative sets could cause many low risk individuals to switch from a generous plan to the HD plan. The removal of low risk individual from the generous plan's risk pool is likely to cause premiums for the generous plan to rise, which in turn could cause more individuals to leave the pool. Furthermore, significant changes in the insurance status of the U.S. population will lead to changes in consumption patterns (moral hazard), which ultimately impacts the prices charged for medical care. In order to make reliable policy predictions, these changes in premiums and medical care prices should be considered. 


\section{REFERENCES}

Akerlof, G. A. (1970). The market for 'lemons': Quality uncertainty and the market mechanism. The Quarterly Journal of Economics 84(3), 488-500.

Aron-Dine, A., L. Einav, and A. Finkelstein (2013). The rand health insurance experiment, three decades later. Journal of Economic Perspectives 27(1), 197-222.

Aron-Dine, A., L. Einav, A. Finkelstein, and M. Cullen (2012). Moral hazard in health insurance: How important is forward looking behavior? NBER Working Paper 17802.

Arrow, K. J. (1963). Uncertainty and the welfare economics of medical care. The American Economic Review 53(5), 941-973.

Baicker, K., S. Taubman, H. Allen, M. Bernstein, J. Gruber, J. P. Newhouse, E. Schneider, B. Wright, A. Zaslavsky, A. Finkelstein, and the Oregon Health Study Group (2013). The oregon experiment - medicaid's effects on clinical outcomes. New England Journal of Medicine 368(18).

Bajari, P., H. Hong, A. Khwaja, and C. Marsh (2013). Moral hazard, adverse selection, and health expenditures: A semiparametric analysis. Working Paper.

Bellman, R. (1957). Dynamic Programming. Princeton University Press.

Berndt, E., B. Hall, R. Hall, and J. Hausman (1974). Estimation and inherence in nonlinear structural models. Annals of Economic and Social Measurement 3(4), 103-116.

Blau, D. and D. Gilleskie (2008). The role of retiree health insurance in the employment behavior of older men. International Economic Review 49(2), 475-514.

Cardon, J. H. and I. Hendel (2001). Asymmetric information in health insurance: Evidence from the national medical expenditure survey. The RAND Journal of Economics 32(3), 408-427.

Chiappori, P. and B. Salanie (2002). Testing contract theory: A survey of some recent work. In M. Dewatripoint, L. Hansen, and P. Turnovsky (Eds.), Advances in Economics and Econometrics - Theory and Applications, Eighth World Congress, pp. 115-149. Cambridge: Cambridge University Press.

Croson, R. and U. Gneezy (2009). Gender differences in preferences. Journal of Economic Literature 47(2), 448-474.

Currie, J. and J. Gruber (1996). Health insurance eligibiity, utilization of medical care, and child health. The Quarterly Journal of Economics 111(2), 432-466.

Cutler, D. and R. Zeckhauser (2000). The anatomy of health insurance. NBER Working Paper 
7176.

Dafny, L. and J. Gruber (2005). Public insurance and child hospitalizations: access and efficiency effects. Journal of Public Economics 89, 109-129.

Dafny, L., K. Ho, and M. Varela (2013). Let them have choice: Gains from shifting away from employer-sponsored health insurance and toward individual exchange. American Economic Journal: Economic Policy 5(1), 32-34.

Dave, D. and R. Kaestner (2009). Health insurance and ex-ante moral hazard. International Journal of Heathcare Finance and Economics 9, 367-390.

de Meza, D. and D. C. Webb (2001). Advantageous selection in insurance markets. The RAND Journal of Economics 32(2), 249-262.

Eckel, C. C. (2008). Men, women and risk aversion: Experimental evidence. Handbook of Experimental Exonomics Results 1, 1062-1073.

Einav, L., A. Finkelstein, and J. Levin (2010). Beyond testin: Empirical models of insurance markets. The Annual Review of Economics 2, 311-336.

Einav, L., A. Finkelstein, S. Ryan, P. Schrimpf, and M. Cullen (2013). Selection on moral hazard in health insurance. American Economic Review 103(1), 178 - 219.

Ellis, R. P. (1986). Rational behavior in the presence of coverage ceilings and deductibles. The RAND Journal of Economics 17(2), 158-175.

Feldman, R. and B. Dowd (1991). A new estimate of the welfare loss of excess health insurance. The American Economic Review 81(1), 297-301.

Feldstein, M. S. (1973). The welfare loss of excess health insurance. The Journal of Political Economy 81(2), 251-280.

Feldstein, M. S. and J. Gruber (1995). A major risk approach to health insurance reform. Tax Policy and the Economy 9, 103-130.

Finkelstein, A. and K. McGarry (2006). Multiple dimensions of private information: Evidence from the long-term care insurance market. American Economic Review 96(4), 938958.

Finkelstein, A. and R. McKnight (2008). What did medicare do? the initial impact of medicare on mortality and out of pocket medical spending. Journal of Public Economics 92, 1644-1668.

Finkelstein, A., S. Taubman, B. Wright, M. Bernstein, J. Gruber, J. P. Newhouse, H. Allen, K. Baicker, and the Oregon Health Study Group (2012). The oregon health insurance 
experiment: Evidence from the first year. Quarterly Hournal of Economics 127(3).

French, E. and J. B. Jones (2011). The effects of health insurance and self-insurance on retirement behavior. Econometrica 79(3), 693-732.

Gilleskie, D. B. (1998). A dynamic stochastic model of medical care use and work absence. Econometrica 66(1), 1-45.

Gilleskie, D. B. and T. A. Mroz (2004). A flexible approach for estimating the effects of covariates on health expenditures. Journal of Health Economics 23(2), 391 - 418.

Greenberg, D. and M. Shroder (2004). The Digest of Social Experiments. Urban Inst Press.

Grossman, M. (1972). On the concept of health capital and the demand for health. Journal of Political Economy 80(2), 223-255.

Guilkey, D. K. and P. M. Lance (2013). Program impact estimation with binary outcome variables: Monte carlo results for alternative estimators and empirical examples. Studies in Applied and Empirical Econometrics A Festschrift in Honor of Peter Schmidt (New York: Springer).

Handel, B. (2013). Adverse selection and switching costs in health insurance markets: When nudging hurts. NBER Working Paper 17459.

Heckman, J. and B. Singer (1984). A method for minimizing the impact of distributional assumptions in econometric models for duration data. Econometrica 52(2), 271-320.

Imai, S. and M. P. Keane (2004). Intertemporal labor supply and human capital accumulation. International Economic Review 45(2), 601 - 641.

Keane, M. P. and K. I. Wolpin (1994). The solution and estimation of discrete choice dynamic programming models by simulation and interpolation: Monte carlo evidence. The Review of Economics and Statistics 76(4), 648-672.

Keeler, E., J. Buchanan, J. Rolph, J. Hanley, and D. Reboussin (1988). The Demand for Episodes of Medical Treatment in the Health Insurance Experiment. Number R-3454HHS. Santa Monica, CA: RAND Publishing.

Keeler, E. B., J. P. Newhouse, and C. E. Phelps (1977). Deductibles and the demand for medical care services: The theory of a consumer facing a variable price schedule under uncertainty. Econometrica 45(3), 641-656.

Keeler, E. B. and J. E. Rolph (1988). The demand for episodes of treatment in the health insurance experiment. Journal of Health Economics 7(4), 337 - 367.

Kelly, I. and S. Markowitz (2009). Incentives in obesity and health insurance. Inquiry 46(4), 
$418-432$.

Khwaja, A. (2001). Health insurance, habits, and health outcomes: Moral hazard in a dynamic stochastic model of investment in health. Working Paper.

Khwaja, A. (2010). Estimating willingness to pay for medicare using a dynamic life-cycle model of demand for health insurance. Journal of Econometrics 156(1), 130 - 147.

Kolstad, J. T. and A. E. Kowalski (2012). The impact of health care reform on hospital and preventive care: Evidence from massachusetts. Journal of Public Economics 96, 909-929.

Kowalski, A. (2013). Estimating the tradeoff between risk protection and moral hazard with a nonlinear budget set model of health insurance. Working Paper.

Manning, W. G. and M. S. Marquis (1996). Health insurance: the tradeoff between risk pooling and moral hazard. Journal of Health Economics 15, 609-639.

Manning, W. G., J. P. Newhouse, N. Duan, E. B. Keeler, and A. Leibowitz (1987). Health insurance and the demand for medical care: Evidence from a randomized experiment. The American Economic Review 77(3), 251-277.

McKenry, L. and E. Salerno (1998). Mosby's Pharmacology in Nursing (20 ed.). St. Louis, MO: Mosby's Publishing.

Miller, S. (2012). The impact of the massachusetts health care reform on health care use among children. American Economic Review: Papers and Proceedings 102(3), 502-507.

Mroz, T. A. (1999). Discrete factor approximations in simultaneous equation models: Estimating the impact of a dummy endogenous variable on a continuous outcome. Journal of Econometrics 92(2), $233-274$.

Mroz, T. A. and D. Guilkey (1992). Discrete factor approximations for use in simultaneous equation models with both continuous and discrete endogenous variables. University of North Carolina Working Paper.

Newhouse, J. P. (1974). A design for a health insurance experiment. Economic Inquiry 11(1).

Newhouse, J. P. (1993). Free For All?: Lessons from the Rand Health Insurance Experiment. Harvard University Press.

Nyman, J. A. (1999a). The economics of moral hazard revisited. Journal of Health Economics 18, 811-824.

Nyman, J. A. (1999b). The value of health insurance: the access motive. Journal of Health Economics 18, 141-152.

Nyman, J. A. (1999c). The welfare economics of insurance contracts that pay off by reducing 
price. Center for Economic Research, Department of Economics, University of Minnesota, Minneapolis.

Pauly, M. V. (1968). The economics of moral hazard: Comment. The American Economic Review 58(3), 531-537.

Rosen, A. B., J. S. Tsai, and S. M. Downs (2003). Variations in risk attitude across race, gender, and education. Medical Decision Making 23, 511-517.

Rust, J. (1987). Optimal replacement of gmc bus engines: An empirical model of harold zurcher. Econometrica 55(5), pp. 999-1033.

Sauer, R. M. (2012). Does it pay for women to volunteer? Working Paper. 